\title{
Aza-Conjugate Addition Methodology for the Synthesis of $N$-Hydroxy-isoindolin-1-ones
}

Santiago Royo, ${ }^{\dagger}$ Robert S. L. Chapman,${ }^{\dagger}$ Alisia M. Sim,${ }^{\dagger}$ Lucy R. Peacock,${ }^{\dagger}$ Steven D. Bull ${ }^{\dagger *}$

$\dagger$ Department of Chemistry, University of Bath, Bath, BA2 7AY, U.K.

† Departament de Química Inorgànica i Orgànica, Universitat Jaume I, Castelló, Spain.

\section{Contents}

S2 General conditions and general procedures

S4 Experimental

S29 $\quad{ }^{1} \mathrm{H}$ and ${ }^{13} \mathrm{C}$ NMR spectra 


\section{General conditions}

Infrared spectra $\left(4000 \mathrm{~cm}^{-1}\right.$ to $\left.0 \mathrm{~cm}^{-1}\right)$ were recorded on a Perkin Elmer Spectrum $100 \mathrm{FT}$-IR spectrometer using a Universal ATR accessory for sampling. The machine has internal calibration and only selected peaks are quoted in $v$ (wavenumbers, $\mathrm{cm}^{-1}$ ).

Proton magnetic resonance spectra were recorded at $300.22 \mathrm{MHz}$ on a Bruker Avance 300 spectrometer unless otherwise stated. Chemical shifts $(\delta \mathrm{H})$ are quoted in parts per million and are referenced to the residual solvent peak. The multiplicities and general assignments of spectroscopic data are denoted as: singlet (s), doublet (d), triplet (t), quartet (q), doublet of doublets (dd), triplet of doublets (td), quartet of doublets (qd), triplet of triplets (tt), multiplet (m), aromatic (Ar), and apparent (app.). Coupling constants $(J)$ are quoted to the nearest $0.1 \mathrm{~Hz}$. Carbon magnetic resonance spectra were recorded at $75.5 \mathrm{MHz}$ on a Bruker Avance 300 spectrometer unless otherwise stated. Chemical shifts $(\delta \mathrm{C})$ are quoted in parts per million and are referenced to the residual solvent peak. Coupling constants $(J)$ are quoted to the nearest 0.1 $\mathrm{Hz}$.

Mass spectra were recorded on a Bruker Daltonics micrOTOF electrospray time-of-flight (ESITOF) mass spectrometer. Samples were introduced either by syringe pump or flow injection using an auto-sampler. Samples were diluted in either methanol or acetonitrile.

All capillary melting point determinations were carried out using Büchi 535 melting point apparatus and reported to the nearest degree Celsius.

Analytical thin layer chromatography was carried out using commercially available polyethylene backed plates coated with Merck Kieselgel 60 GF254. Plates were visualised under UV light (at $254 \mathrm{~nm}$ ) or by staining with potassium permanganate, p-anisaldehyde or phosphomolybdic acid followed by heating. Flash chromatography was performed under medium pressure using Merck $60 \mathrm{H}$ silica gel $(35-75 \mu \mathrm{m})$. Samples were loaded as saturated solutions in an appropriate solvent.

Reactions requiring anhydrous conditions were performed under nitrogen in oven-dried apparatus, which was allowed to cool under nitrogen prior to use. Anhydrous solvents were obtained by passing through anhydrous alumina columns using an Innovative Technology Inc. PS-400-7 solvent purification system. Petrol refers to the fraction of petroleum ether boiling at $40-60{ }^{\circ} \mathrm{C}$. Ether refers to diethyl ether. Hexanes refer to the hexane fraction of petroleum. Solvents were evaporated on a Büchi Rotorvapor.

All commercially available compounds were used as obtained from the chemical suppliers. All temperatures quoted are external 


\section{General Procedures}

\section{General procedure A1: Synthesis of Sonigashira cross coupled products}

2-Bromobenzaldehyde (1 eq) in $\mathrm{Et}_{3} \mathrm{~N}(15 \mathrm{~mL} / \mathrm{g}$ ) was stirred and degassed (under a stream of nitrogen) for 10 minutes. Ethynyltriisopropylsilane (1.2 eq) was then added to this solution, followed by triphenylphosphine $(0.04 \mathrm{eq})$ and palladium acetate $(0.02 \mathrm{eq})$. The reaction was stirred under nitrogen at $80{ }^{\circ} \mathrm{C}$ for $16 \mathrm{~h}$. The reaction was cooled and diluted with $\mathrm{Et}_{2} \mathrm{O}(25 \mathrm{~mL})$. The solid was filtered and the solution was washed with saturated $\mathrm{NaHCO}_{3}(25 \mathrm{~mL}$ x 3$)$ and water $(25 \mathrm{~mL}$ x 3$)$. The organic layer was dried over $\mathrm{MgSO}_{4}$, filtered and concentrated in vасио to give the crude product. Purification by flash column chromatography afforded the title compound.

\section{General procedure A2: Synthesis of Sonigashira cross coupled products}

2-Bromobenzaldehyde (1 eq) in $\mathrm{Et}_{3} \mathrm{~N}(10 \mathrm{~mL} / \mathrm{g})$ and DMF $(1 \mathrm{~mL} / \mathrm{g})$ was stirred and degassed (under a stream of nitrogen) for 10 minutes. Ethynyltriisopropylsilane (1.2eq) was then added to this solution, followed by triphenylphosphine (0.04 eq) and palladium acetate $(0.02 \mathrm{eq})$. The reaction was stirred under a nitrogen atomosphere at $80^{\circ} \mathrm{C}$ for $16 \mathrm{~h}$. The reaction mixture was cooled and diluted with $\mathrm{Et}_{2} \mathrm{O}(25 \mathrm{~mL})$. The solid was filtered-off and the solution was washed with saturated $\mathrm{NaHCO}_{3}(25 \mathrm{~mL}$ x3) and water $(25$ $\mathrm{mL} \times 3$ ). The organic layer was dried with $\mathrm{MgSO}_{4}$ and concentrated in vacuo to give the crude product. Purification by flash column chromatography afforded the title compound.

\section{General procedure B: Synthesis of Acetal products}

1,3-Propandiol (1.5 eq) and $p$-toluenesulfonic acid monohydrate ( $0.1 \mathrm{eq})$ were added to a stirred solution of benzaldehyde $(1 \mathrm{eq})$ in toluene $(10 \mathrm{~mL} / \mathrm{g})$, at room temperature. The solution was heated to $90{ }^{\circ} \mathrm{C}$ for $4.5 \mathrm{~h}$, at which point TLC analysis indicated complete consumption of starting material. The solution was cooled to room temperature and washed with saturated $\mathrm{NaHCO}_{3}(20 \mathrm{~mL}$ x 3). The organic layer were dried with $\mathrm{MgSO}_{4}$ and concentrated in vacuo to give the title compound that was used in the subsequent step without further purification.

\section{General procedure C: Synthesis of acetal methyl propiolate products}

TBAF (1M in THF) (2 eq) was added to a solution of triisopropylsilane $(1 \mathrm{eq})$ in $\mathrm{Et}_{2} \mathrm{O}(10 \mathrm{~mL} / \mathrm{g})$ stirred under a nitrogen atmosphere at $0{ }^{\circ} \mathrm{C}$. The mixture was stirred at $0{ }^{\circ} \mathrm{C}$ for 10 minutes and then allowed to warm up to $\mathrm{rt}$ and stirred for $1 \mathrm{hr}$. The reaction was then diluted with $\mathrm{Et}_{2} \mathrm{O}$ and washed with $\mathrm{H}_{2} \mathrm{O}(2 \mathrm{x} 20$ $\mathrm{mL})$, brine $(2 \times 20 \mathrm{~mL})$ and dried $\mathrm{MgSO}_{4}$. The reaction was concentrated in vacuo. The residue was disolved in anhydrous THF $(10 \mathrm{~mL} / \mathrm{g})$ and cooled to $-78{ }^{\circ} \mathrm{C}$, followed by dropwise addition of $n$-BuLi (1.6 M) (3 eq), The resultant solution stirred for $1 \mathrm{~h}$ maintaining the temperature at below $-66^{\circ} \mathrm{C}$. A precooled solution of methyl chloroformate (6 eq) was then added as fast as possible. After addition the reaction was left to warm to room temperature over $1 \mathrm{~h}$. The reaction was extracted with $\mathrm{Et}_{2} \mathrm{O}$, washed with $\mathrm{H}_{2} \mathrm{O}(2 \times 20 \mathrm{~mL})$, 
followed by brine $(2 \times 20 \mathrm{~mL})$ and then dried $\left(\mathrm{MgSO}_{4}\right)$. The reaction was concentrated in vacuo and the residue purified via column chromatography.

\section{General procedure D: Synthesis of aldehyde methyl propiolate products}

Concentrated $\mathrm{HCl} 36 \%$ (60 eq) was added dropwise to a solution of acetal propiolate (1 eq) in THF $(5 \mathrm{ml} / \mathrm{mmol})$ at $0{ }^{\circ} \mathrm{C}$ and the resultant solution allowed to warm up to $\mathrm{rt}$ and stirred for $4 \mathrm{~h}$. The reaction was then diluted with $\mathrm{Et}_{2} \mathrm{O}(20 \mathrm{~mL})$ and subsequently quenched with solid $\mathrm{NaHCO}_{3}$ and saturated $\mathrm{NaHCO}_{3}$. When a neutral $\mathrm{pH}$ was reached the two layers were separated and the aqueous layer was further extracted with $\mathrm{Et}_{2} \mathrm{O}\left(15 \mathrm{~mL}\right.$ x 3). The organic layers were combined, dried with $\mathrm{MgSO}_{4}$ and concentrated in vacuo to give the title compound that was used in the subsequent step without further purification.

\section{General procedure E: Synthesis of hydroxamic acid products}

Hydroxylamine solution $50 \%$ in $\mathrm{H}_{2} \mathrm{O} \quad(1.1$ eq $)$ was added to a solution of methyl 3-(2formylphenyl)propiolate (1 eq) in THF $(10 \mathrm{~mL} / \mathrm{g})$ at room temperatura and stirred for $1 \mathrm{hr}$. The solution was concentrated in vacuo and the residue was purified via column chromatography (5\% $\mathrm{MeOH}$ in DCM).

\section{General route for the synthesis of cyclic hydroxamic acids}<smiles>O=Cc1c[R]#ccc1Br</smiles>

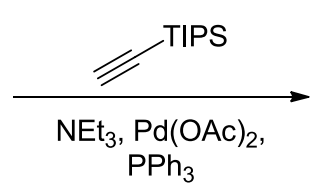<smiles>O=Cc1c[R]ccc1C#C[In]</smiles><smiles>OCCCO</smiles><smiles>C(#Cc1ccccc1C1OCCCO1)c1ccccc1</smiles>

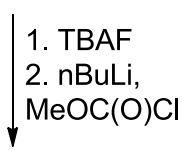<smiles>COC(=O)CC1c2cc[R]cc2C(=O)N1O</smiles><smiles>CC(=O)C#Cc1cc[R](CCN)cc1C=O</smiles><smiles>CC1CCCC1</smiles><smiles></smiles>

\section{Experimental data}

\section{Synthesis of 2-((triisopropylsilyl)ethynyl)benzaldehyde 6a}<smiles>CC(C)[Si](C#Cc1ccccc1C=O)(C(C)C)C(C)C</smiles>

General procedure A1 was followed using 2-bromobenzaldehyde (2.0 mL, $16.79 \mathrm{mmol})$. Purification by flash column chromatography (2.5\% EtOAc in pentane) afforded the title compound ( $3.971 \mathrm{~g}, 83 \%)$ as a 
pale yellow oil; ${ }^{1} \mathrm{H}$ NMR (300MHz, $\left.\mathrm{CDCl}_{3}\right) \delta$ ppm 10.60 (s, 1H, CHO), 7.89 (dd, J=7.7, $\left.1.4 \mathrm{~Hz}, 1 \mathrm{H}, \mathrm{ArH}\right)$, $7.57(\mathrm{dd}, J=7.7,1.4 \mathrm{~Hz}, 1 \mathrm{H}, \mathrm{ArH}), 7.51(\mathrm{dd}, J=7.7,1.4 \mathrm{~Hz}, 1 \mathrm{H}, \mathrm{ArH}), 7.40$ (ddd, $J=8.0,1.4,0.7 \mathrm{~Hz}$, $1 \mathrm{H}, \mathrm{ArH}), 1.12$ (d, $J=2.3 \mathrm{~Hz}, 21 \mathrm{H}, \mathrm{TIPS}) ;{ }^{13} \mathrm{C} \mathrm{NMR}\left(75 \mathrm{MHz}, \mathrm{CDCl}_{3}\right) \delta \mathrm{ppm} 191.7,136.3,133.9,133.7$, 128.7, 127.2, 126.9, 102.1, 99.1, 18.7, 11.3; IR (thin film) $v_{\max }\left(\mathrm{cm}^{-1}\right): 2154.49(\mathrm{C} \equiv \mathrm{C}), 1699.56(\mathrm{C}=\mathrm{O})$; HRMS (ESI): $\mathrm{m} / \mathrm{z}$ calculated for $\mathrm{C}_{18} \mathrm{H}_{26} \mathrm{OSi}$ : 286.1753; for $[\mathrm{M}+\mathrm{H}]^{+}$found: 286.1818 ; for $[\mathrm{M}+\mathrm{Na}]^{+}$found 309.1626.

\section{Synthesis of 6-((triisopropylsilyl)ethynyl)benzo[d][1,3]dioxole-5-carbaldehyde $6 \mathrm{~b}$}

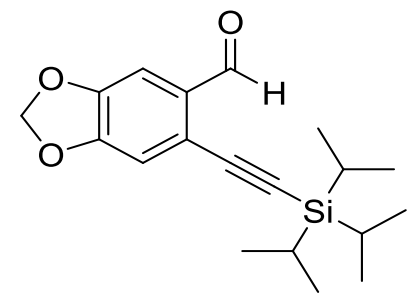

General procedure A2 was followed using 6-bromopiperonal (2.078 g, $8.89 \mathrm{mmol})$. Purification by flash column chromatography (5\% EtOAc in pentane) on silica afforded the product $(2.56 \mathrm{~g}, 87 \%)$ as a pale yellow solid. mp 52-54 ${ }^{\circ} \mathrm{C}$; ${ }^{1} \mathrm{H}$ NMR $\left(300 \mathrm{MHz}, \mathrm{CDCl}_{3}\right) \delta \mathrm{ppm} 10.43$ (s, 1H, CHO), 7.32 (s, 1H, ArH), 6.96 $(\mathrm{s}, 1 \mathrm{H}, \mathrm{ArH}), 6.06\left(\mathrm{~s}, 2 \mathrm{H}, \mathrm{OCH}_{2} \mathrm{O}\right), 1.13(\mathrm{~d}, J=2.5 \mathrm{~Hz}, 21 \mathrm{H}, \mathrm{TIPS}) ;{ }^{13} \mathrm{C} \mathrm{NMR}\left(75 \mathrm{MHz}, \mathrm{CDCl}_{3}\right) \delta \mathrm{ppm}$ 190.2, 152.4, 148.9, 132.8, 124.0, 112.8, 106.0, 102.5, 102.0, 98.0, 18.8, 11.4; IR (thin film) $v_{\max }\left(\mathrm{cm}^{-1}\right)$ : $2154.64(\mathrm{C} \equiv \mathrm{C}), 1680.82(\mathrm{C}=\mathrm{O}), 1261.87$ and $1025.34\left(\mathrm{OCH}_{2}\right) ; \mathrm{HRMS}(\mathrm{ESI}): \mathrm{m} / \mathrm{z}$ calculated for $\mathrm{C}_{19} \mathrm{H}_{26} \mathrm{O}_{3} \mathrm{Si}: 331.1651$; for $[\mathrm{M}+\mathrm{H}]^{+}$found: 331.1713 ; for $[\mathrm{M}+\mathrm{Na}]^{+}$found 353.1553 .

\section{Synthesis of 5-(trifluoromethyl)-2-((triisopropylsilyl)ethynyl)benzaldehyde 6c}

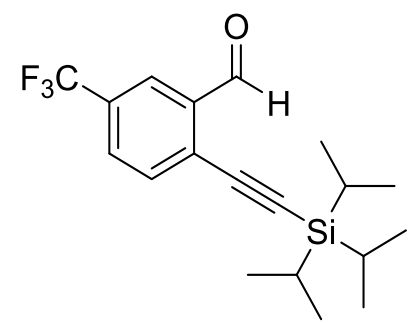

General procedure A1 was followed using 2-bromo-5-(trifluoromethyl)benzaldehyde (1.0 mL, $6.63 \mathrm{mmol})$. Purification by flash column chromatography (2.5\% EtOAc in pentane) afforded the title compound (1.96 $\mathrm{g}, 83 \%)$ as a clear oil; ${ }^{1} \mathrm{H} \mathrm{NMR}\left(300 \mathrm{MHz}, \mathrm{CDCl}_{3}\right) \delta \mathrm{ppm} 10.61(\mathrm{~s}, 1 \mathrm{H}, \mathrm{CHO}), 8.17(\mathrm{~d}, J=1.7 \mathrm{~Hz}, 1 \mathrm{H}$, ArH), $7.77(\mathrm{dd}, J=8.2,1.7 \mathrm{~Hz}, 1 \mathrm{H}, \mathrm{ArH}), 7.71(\mathrm{~d}, J=8.1 \mathrm{~Hz}, 1 \mathrm{H}, \mathrm{ArH}), 1.14(\mathrm{~d}, \mathrm{~J}=3.9 \mathrm{~Hz}, 21 \mathrm{H}, \mathrm{TIPS}) .{ }^{13} \mathrm{C}$ $\operatorname{NMR}\left(75 \mathrm{MHz}, \mathrm{CDCl}_{3}\right) \delta \mathrm{ppm} 190.4,136.5,134.6,130.9\left(\mathrm{q},{ }^{2} J \mathrm{C}-\mathrm{F}=33.6 \mathrm{~Hz}\right), 130.3,130.0\left(\mathrm{q},{ }^{3} J \mathrm{C}-\mathrm{F}=\right.$ $3.5 \mathrm{~Hz}), 124.2\left(\mathrm{q},{ }^{3} \mathrm{JC}-\mathrm{F}=3.8 \mathrm{~Hz}\right), 123.4\left(\mathrm{q},{ }^{1} J \mathrm{C}-\mathrm{F}=272.6 \mathrm{~Hz}\right), 102.8,100.7,18.7,11.3$; IR (thin film) $v_{\max }\left(\mathrm{cm}^{-1}\right): 2160.51(\mathrm{C} \equiv \mathrm{C}), 1695.81(\mathrm{C}=\mathrm{O}), 1327.76,1174.97$ and $1138.97\left(\mathrm{CF}_{3}\right) ; \mathrm{HRMS}(\mathrm{ESI}): \mathrm{m} / \mathrm{z}$ calculated for $\mathrm{C}_{19} \mathrm{H}_{25} \mathrm{~F}_{3} \mathrm{OSi}$ : 355.1627 ; for $[\mathrm{M}+\mathrm{H}]^{+}$found: 355.1715 ; for $[\mathrm{M}+\mathrm{Na}]^{+}$found 377.1525 
Synthesis of 5-fluoro-2-((triisopropylsilyl)ethynyl)benzaldehyde 6d

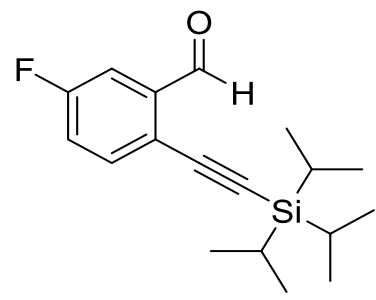

General procedure A1 was followed using 2-bromo-5-fluorobenzaldehyde (2.25 g, $11.08 \mathrm{mmol})$. Purification by flash column chromatography (2.5\% EtOAc in pentane) on silica afforded the title compound $(3.07 \mathrm{~g}, 91 \%)$ as a pale yellow oil; ${ }^{1} \mathrm{H}$ NMR $\left(300 \mathrm{MHz}, \mathrm{CDCl}_{3}\right) \delta \mathrm{ppm} 10.55(\mathrm{~d}, J=3.3 \mathrm{~Hz}, 1 \mathrm{H}$, CHO), 7.58 (dt, $J=8.5,4.3 \mathrm{~Hz}, 2 \mathrm{H}, \mathrm{ArH}), 7.25$ (td, $J=8.1,2.8 \mathrm{~Hz}, 1 \mathrm{H}, \mathrm{ArH}), 1.13(\mathrm{~d}, J=3.0 \mathrm{~Hz}, 21 \mathrm{H}$, TIPS); ${ }^{13} \mathrm{C}$ NMR $\left(75 \mathrm{MHz}, \mathrm{CDCl}_{3}\right) \delta \mathrm{ppm} 190.6\left(\mathrm{~d},{ }^{4} \mathrm{JC}-\mathrm{F}=1.5 \mathrm{~Hz}\right), 162.5\left(\mathrm{~d},{ }^{1} \mathrm{JC}-\mathrm{F}=252.8 \mathrm{~Hz}\right), 138.4(\mathrm{~d}$, $\left.{ }^{3} J \mathrm{C}-\mathrm{F}=6.7 \mathrm{~Hz}\right), 136.1\left(\mathrm{~d},{ }^{3} \mathrm{JC}-\mathrm{F}=7.6 \mathrm{~Hz}\right), 123.3\left(\mathrm{~d},{ }^{4} \mathrm{JC}-\mathrm{F}=3.4 \mathrm{~Hz}\right), 121.4\left(\mathrm{~d},{ }^{2} J \mathrm{C}-\mathrm{F}=22.8 \mathrm{~Hz}\right), 113.5$ $\left(\mathrm{d},{ }^{2} \mathrm{JC}-\mathrm{F}=23.0 \mathrm{~Hz}\right), 101.04,99.1,18.8,11.4$; IR (thin film) $v_{\max }\left(\mathrm{cm}^{-1}\right): 2213.22(\mathrm{C} \equiv \mathrm{C}), 1706.89(\mathrm{C}=\mathrm{O})$; HRMS (ESI): m/z calculated for $\mathrm{C}_{18} \mathrm{H}_{25} \mathrm{OFSi}$ : 305.1659; for $[\mathrm{M}+\mathrm{H}]^{+}$found: 305.1713 ; for $[\mathrm{M}+\mathrm{Na}]^{+}$found 327.1531 .

\section{Synthesis of 4,5-dimethoxy-2-((triisopropylsilyl)ethynyl)benzaldehyde 6e}<smiles>COc1cc(C#C[Si](C(C)C)(C(C)C)C(C)C)c(C=O)cc1OC</smiles>

General procedure A2 was followed using 2-bromo-4,5-dimethoxybenzaldehyde (2.021 g, $8.08 \mathrm{mmol})$. Purified by column chromatography (10\% EtOAc in pentane) affored the title compound (2.46 g, 85\%) as a yellow solid. mp $95-97{ }^{\circ} \mathrm{C} ;{ }^{1} \mathrm{H}$ NMR $\left(300 \mathrm{MHz}, \mathrm{CDCl}_{3}\right) \delta \mathrm{ppm} 10.45$ (s, 1H, CHO), $7.38(\mathrm{~s}, 1 \mathrm{H}, \mathrm{ArH})$, $6.96(\mathrm{~s}, 1 \mathrm{H}, \mathrm{ArH}), 3.97\left(\mathrm{~s}, 3 \mathrm{H}, \mathrm{OCH}_{3}\right), 3.93\left(\mathrm{~s}, 3 \mathrm{H}, \mathrm{OCH}_{3}\right), 1.13(\mathrm{~d}, J=2.8 \mathrm{~Hz}, 21 \mathrm{H}, \mathrm{TIPS}) ;{ }^{13} \mathrm{C}$ NMR $(75$ $\left.\mathrm{MHz}, \mathrm{CDCl}_{3}\right) \delta$ ppm 190.5, 153.6, 149.8, 130.7, 121.8, 114.7, 108.0, 101.9, 97.4, 56.3, 56.1, 18.7, 11.3; IR (thin film) $v_{\max }\left(\mathrm{cm}^{-1}\right): 2143.71(\mathrm{C} \equiv \mathrm{C}), 1680.07(\mathrm{C}=\mathrm{O}), 1276.70$ and $1106.57\left(\mathrm{OCH}_{3}\right) ; \mathrm{HRMS}(\mathrm{ESI}): \mathrm{m} / \mathrm{z}$ calculated for $\mathrm{C}_{20} \mathrm{H}_{30} \mathrm{O}_{3} \mathrm{Si}$ : 347.1964 ; for $[\mathrm{M}+\mathrm{H}]^{+}$found: 347.2041

\section{Synthesis of 5-methoxy-2-((triisopropylsilyl)ethynyl)benzaldehyde of}<smiles>COc1ccc(C#C[Si](C(C)C)(C(C)C)C(C)C)c(C=O)c1</smiles> 
General procedure A2 was followed using 2-bromo-5-methoxybenzaldehyde (2.041 g, $9.49 \mathrm{mmol})$. Purified by flash column chromatography (3\% EtOAc in pentane) afforded the title compound ( $2.70 \mathrm{~g}$, 90\%) as a yellow oil; ${ }^{1} \mathrm{H}$ NMR (300 MHz, $\left.\mathrm{CDCl}_{3}\right) \delta \mathrm{ppm} 10.56(\mathrm{~s}, 1 \mathrm{H}, \mathrm{CHO}), 7.50(\mathrm{~d}, J=8.6 \mathrm{~Hz}, 1 \mathrm{H}$, $\mathrm{ArH}), 7.37(\mathrm{~d}, J=2.8 \mathrm{~Hz}, 1 \mathrm{H}, \mathrm{ArH}), 7.07(\mathrm{dd}, J=8.5,2.8 \mathrm{~Hz}, 1 \mathrm{H}, \mathrm{ArH}), 3.84\left(\mathrm{~s}, 3 \mathrm{H}, \mathrm{OCH}_{3}\right), 1.12(\mathrm{~d}, J=$ $1.8 \mathrm{~Hz}, 21 \mathrm{H}, \mathrm{TIPS}) .{ }^{13} \mathrm{C} \mathrm{NMR}\left(75 \mathrm{MHz}, \mathrm{CDCl}_{3}\right) \delta \mathrm{ppm} 191.7,159.9,137.8,135.3,121.7,120.0,109.5$, 102.1, 97.2, 55.7, 18.8, 11.4; IR (thin film) $v_{\max }\left(\mathrm{cm}^{-1}\right): 2151.17(\mathrm{C} \equiv \mathrm{C}), 1697.88(\mathrm{C}=\mathrm{O}), 1277.86$ and $1032.43\left(\mathrm{OCH}_{3}\right)$; HRMS (ESI): $\mathrm{m} / \mathrm{z}$ calculated for $\mathrm{C}_{19} \mathrm{H}_{28} \mathrm{O}_{2} \mathrm{Si}$ : 317.1859; for [M+H] ${ }^{+}$found: 317.1928 ; for $[\mathrm{M}+\mathrm{Na}]^{+}$found: 339.1749

Synthesis of 2-fluoro-6-(2-(triisopropylsilyl)ethynyl)benzaldehyde $6 \mathrm{~g}$

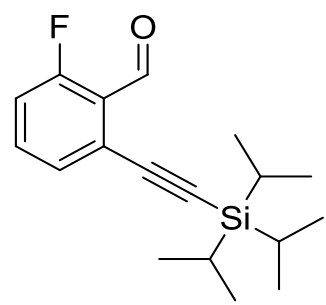

General procedure A1 was followed uysing 2-bromo-6-fluorobenzaldehyde (1.91 g, $9.41 \mathrm{mmol})$. Purification by flash column chromatography (2\% EtOAc in pentane) on silica afforded the product (2.04 g, 91\%) as a yellow oil oil; ${ }^{1} \mathrm{H}$ NMR (500 MHz, $\left.\mathrm{CDCl}_{3}\right) \delta \mathrm{ppm} 10.57$ (d, $\left.J=0.4 \mathrm{~Hz}, 1 \mathrm{H}, \mathrm{CHO}\right), 7.54-7.44$ (m, 1H, ArH), $7.42-7.36$ (m, 1H, ArH), $7.15-7.06$ (m, 1H, ArH), $1.18-1.09$ (m, 21H, TIPS); ${ }^{13} \mathrm{C}$ NMR $\left(75 \mathrm{MHz}, \mathrm{CDCl}_{3}\right) \delta \mathrm{ppm} 188.9,162.0\left(\mathrm{~d},{ }^{1} J_{\mathrm{C}-\mathrm{F}}=263.9 \mathrm{~Hz}\right), 134.9\left(\mathrm{~d},{ }^{3} J_{\mathrm{C}-\mathrm{F}}=10.5 \mathrm{~Hz}\right), 130.3\left(\mathrm{~d},{ }^{4} J_{\mathrm{C}-\mathrm{F}}=\right.$ $3.7 \mathrm{~Hz}), 128.1\left(\mathrm{~d},{ }^{3} J_{\mathrm{C}-\mathrm{F}}=3.4 \mathrm{~Hz}\right), 124.6\left(\mathrm{~d},{ }^{2} J_{\mathrm{C}-\mathrm{F}}=8.0 \mathrm{~Hz}\right), 117.2\left(\mathrm{~d},{ }^{2} J_{\mathrm{C}-\mathrm{F}}=21.4 \mathrm{~Hz}\right), 101.6\left(\mathrm{~d},{ }^{4} J_{\mathrm{C}-\mathrm{F}}=\right.$ $4.0 \mathrm{~Hz}), 100.6,18.7$, 11.3; IR (thin film) $v_{\max }\left(\mathrm{cm}^{-1}\right): 2154.14(\mathrm{C} \equiv \mathrm{C}), 1701.97(\mathrm{C}=\mathrm{O}) ; \mathrm{HRMS}(\mathrm{ESI}): \mathrm{m} / \mathrm{z}$ calculated for $\mathrm{C}_{18} \mathrm{H}_{25} \mathrm{OFSi}$ : 305.1659 ; for $[\mathrm{M}+\mathrm{H}]^{+}$found: 305.1690 ; for $[\mathrm{M}+\mathrm{Na}]^{+}$found: 327.1498 .

\section{Synthesis of ((2-(1,3-dioxan-2-yl)phenyl)ethynyl)triisopropylsilane}

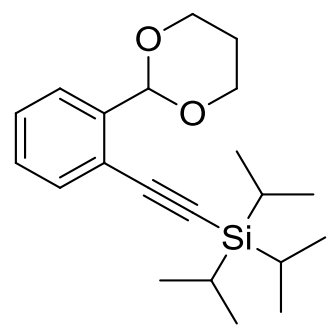

General procedure B was followed using 2-((triisopropylsilyl)ethynyl)benzaldehyde 6a (3.970 g, 13.86 mmol, $1 \mathrm{eq})$ affording the title compound $(4.37 \mathrm{~g}, 92 \%)$ as a pale yellow oil that was used in the subsequent step without further purification; ${ }^{1} \mathrm{H}$ NMR $\left(500 \mathrm{MHz}, \mathrm{CDCl}_{3}\right) \delta \mathrm{ppm} 7.71-7.67(\mathrm{~m}, 1 \mathrm{H}, \mathrm{ArH}), 7.49$ (dd, $J$ = 7.7, $1.3 \mathrm{~Hz}, 1 \mathrm{H}, \mathrm{ArH}), 7.36$ (td, $J=7.6,1.3 \mathrm{~Hz}, 1 \mathrm{H}, \mathrm{ArH}), 7.28(\mathrm{td}, J=7.6,1.4 \mathrm{~Hz}, 1 \mathrm{H}, \mathrm{ArH}), 6.02(\mathrm{~s}$, $1 \mathrm{H}, \mathrm{ArCH}$ ), 4.27 (ddd, $J=11.8,4.9,1.1 \mathrm{~Hz}, 2 \mathrm{H}, \mathrm{OCH}_{2}$ ), 3.99 (ddd, $\left.J=12.4,3.9,2.5 \mathrm{~Hz}, 2 \mathrm{H}, \mathrm{OCH}_{2}\right), 2.33$ $-2.20\left(\mathrm{~m}, 1 \mathrm{H}, \mathrm{CH}_{2} \mathrm{CH}_{\mathrm{A}} \mathrm{CH}_{\mathrm{B}} \mathrm{CH}_{2}\right), 1.44\left(\mathrm{dtt}, J=13.4,2.5,1.3 \mathrm{~Hz}, 1 \mathrm{H}, \mathrm{CH}_{2} \mathrm{CH}_{\mathrm{A}} \mathrm{CH}_{\mathrm{B}} \mathrm{CH}_{2}\right), 1.18(\mathrm{~d}, J=7.9$ $\mathrm{Hz}, 21 \mathrm{H}, \mathrm{TIPS}) .{ }^{13} \mathrm{C}$ NMR $\left(126 \mathrm{MHz}, \mathrm{CDCl}_{3}\right) \delta \mathrm{ppm} \mathrm{140.2,} \mathrm{132.8,} \mathrm{128.8,} \mathrm{128.6,} \mathrm{126.0,} \mathrm{122.0,} \mathrm{104.3,} \mathrm{100.3,}$ 
95.1, 67.8, 26.0, 18.9, 11.5; IR (thin film) $v_{\max }\left(\mathrm{cm}^{-1}\right): 2154.97$ (C $\left.\equiv \mathrm{C}\right)$; HRMS (ESI): m/z $\mathrm{C}_{21} \mathrm{H}_{32} \mathrm{O}_{2} \mathrm{Si}$ calculated for $[\mathrm{M}+\mathrm{H}]^{+}$345.224983, found 345.2182

\section{Synthesis of ((6-(1,3-dioxan-2-yl)benzo[d][1,3]dioxol-5-yl)ethynyl)triisopropylsilane}

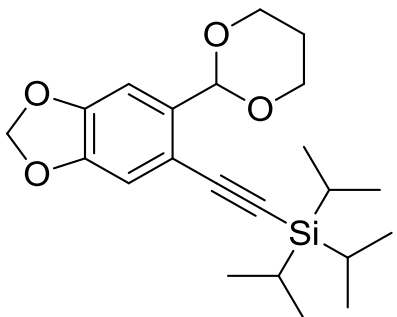

General procedure B was followed using 6-((triisopropylsilyl)ethynyl)benzo[d][1,3]dioxole-5carbaldehyde 6b (2.562 g, $7.75 \mathrm{mmol})$. Column chromatography of the crude (5\% EtOAc in pentane) afforded the title compound $(2.842 \mathrm{~g}, 94 \%)$ as a white solid. mp 61-64 ${ }^{\circ} \mathrm{C} ;{ }^{1} \mathrm{H} \mathrm{NMR}\left(250 \mathrm{MHz}, \mathrm{CDCl}_{3}\right) \delta$ ppm 7.14 ( s, 1H, ArH), 6.89 (s, 1H, ArH), 5.95 (s, 1H, ArCH), 5.93 (s, 2H, OCH $2 \mathrm{O}), 4.23$ (dd, J=11.3, 4.4 $\mathrm{Hz}, 2 \mathrm{H}, \mathrm{OCH}_{2}$ ), 3.95 (td, $J=12.4,2.4, \mathrm{~Hz}, 2 \mathrm{H}, \mathrm{OCH}_{2}$ ), 2.21 (qt, $J=12.5,5.0 \mathrm{~Hz}, 1 \mathrm{H}, \mathrm{CH}_{2} \mathrm{CH}_{\mathrm{A}} \mathrm{CH}_{\mathrm{B}} \mathrm{CH}_{2}$ ) 1.41 (ddd, $\left.J=12.3,2.5,1.2 \mathrm{~Hz}, 1 \mathrm{H}, \mathrm{CH}_{2} \mathrm{CH}_{\mathrm{A}} \mathrm{CH}_{\mathrm{B}} \mathrm{CH}_{2}\right), 1.28$ (m, 21H, TIPS); ${ }^{13} \mathrm{C}$ NMR $\left(75 \mathrm{MHz}, \mathrm{CDCl}_{3}\right) \delta \mathrm{ppm}$ $148.4,147.6,135.7,115.4,111.8,106.7,104.2$, 101.5, 100.0, 93.4, 67.7, 25.8, 18.8, 11.4; IR (thin film) $v_{\max }\left(\mathrm{cm}^{-1}\right): 2148.03(\mathrm{C} \equiv \mathrm{C}), 1256.18$ and $1094.75\left(\mathrm{OCH}_{2}\right)$; HRMS (ESI): m/z calculated for $\mathrm{C}_{22} \mathrm{H}_{32} \mathrm{O}_{4} \mathrm{Si}$ : 389.2070 for $[\mathrm{M}+\mathrm{H}]^{+}$; found: $411.1979[\mathrm{M}+\mathrm{Na}]^{+}$.

\section{Synthesis of ((2-(1,3-dioxan-2-yl)-4-(trifluoromethyl)phenyl)ethynyl)triisopropylsilane}

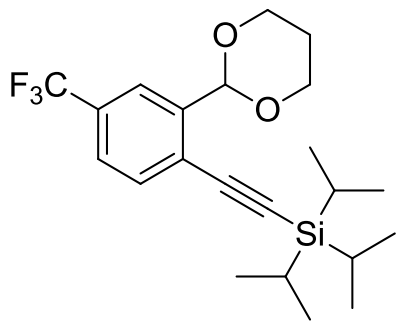

General procedure B was followed using 5-(trifluoromethyl)-2-((triisopropylsilyl)ethynyl)benzaldehyde 6c $(1.950 \mathrm{~g}, 5.50 \mathrm{mmol})$. Concentrated in vacuo to give the title compound as a pale yellow solid (1.832 $\mathrm{g}$, $81 \%)$ that was used in the subsequent step without further purification. mp 80-84 ${ }^{\circ} \mathrm{C} ;{ }^{1} \mathrm{H} \mathrm{NMR}(300 \mathrm{MHz}$, $\left.\mathrm{CDCl}_{3}\right) \delta \mathrm{ppm} 7.99(\mathrm{~s}, 1 \mathrm{H}, \mathrm{ArH}), 7.59$ (d, J=8.1Hz, 1H, ArH), 7.53 (dd, J=8.3, 1.4 Hz, 1H, ArH), 6.01 (s, $1 \mathrm{H}, \mathrm{ArCH}), 4.29$ (dd, $\left.J=11.2,4.9 \mathrm{~Hz}, 2 \mathrm{H}, \mathrm{OCH}_{2}\right), 4.00$ (td, $\left.J=12.3,2.1,2 \mathrm{H}, \mathrm{OCH}_{2}\right), 2.37-2.18(\mathrm{~m}, 1 \mathrm{H}$, $\mathrm{CH}_{2} \mathrm{CH}_{\mathrm{A}} \mathrm{CH}_{\mathrm{B}} \mathrm{CH}_{2}$ ), 1.46 (d, J=13.5 Hz, $\left.1 \mathrm{H}, \mathrm{CH}_{2} \mathrm{CH}_{\mathrm{A}} \mathrm{CH}_{\mathrm{B}} \mathrm{CH}_{2}\right), 1.19$ ( s, 21H, TIPS); ${ }^{13} \mathrm{C}$ NMR $(75 \mathrm{MHz}$, $\left.\mathrm{CDCl}_{3}\right) \delta \mathrm{ppm} 140.8,133.0,130.4\left(\mathrm{q},{ }^{2} \mathrm{JC}-\mathrm{F}=32.8 \mathrm{~Hz}\right), 125.5\left(\mathrm{q},{ }^{4} J \mathrm{C}-\mathrm{F}=1.2 \mathrm{~Hz}\right), 125.3\left(\mathrm{q},{ }^{3} J \mathrm{C}-\mathrm{F}=3.7\right.$ $\mathrm{Hz}), 123.9$ (q, $\left.{ }^{1} \mathrm{JC}-\mathrm{F}=272.4 \mathrm{~Hz}\right), 123.4\left(\mathrm{q},{ }^{3} \mathrm{JC}-\mathrm{F}=3.9 \mathrm{~Hz}\right), 102.7,99.3,98.3,67.7,25.7,18.7,11.3$; IR (thin film) $v_{\max }\left(\mathrm{cm}^{-1}\right): 2160.95(\mathrm{C} \equiv \mathrm{C}), 1325.94,1125.06$ and $1096.44\left(\mathrm{CF}_{3}\right) ;$ HRMS $(\mathrm{ESI}): \mathrm{m} / \mathrm{z}$ calculated for $\mathrm{C}_{22} \mathrm{H}_{31} \mathrm{~F}_{3} \mathrm{O}_{2} \mathrm{Si}$ : 413.2045; for $[\mathrm{M}+\mathrm{H}]^{+}$found: 413.2102; for $[\mathrm{M}+\mathrm{Na}]^{+}$found 435.1925 . 
Synthesis of ((2-(1,3-dioxan-2-yl)-4-fluorophenyl)ethynyl)triisopropylsilane

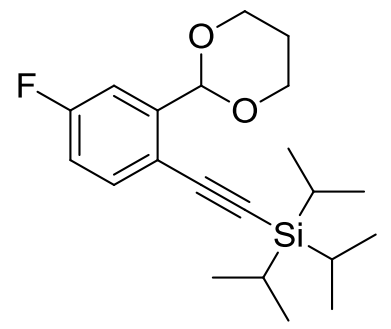

General procedure B was followed using 5-fluoro-2-((triisopropylsilyl)ethynyl)benzaldehyde 6d (3.07 g, $10.08 \mathrm{mmol})$. Concentrated in vacuo to give the title compound as an orange oil $(3.21 \mathrm{~g}, 88 \%)$ that was used in the subsequent step without further purification; ${ }^{1} \mathrm{H}$ NMR $\left(300 \mathrm{MHz}, \mathrm{CDCl}_{3}\right) \delta \mathrm{ppm} 7.46(\mathrm{dd}, J=$ 8.6, 5.5 Hz, 1H, ArH), 7.39 (dd, $J=9.5,2.7 \mathrm{~Hz}, 1 \mathrm{H}, \mathrm{ArH}), 6.97(\mathrm{td}, J=8.3,2.8 \mathrm{~Hz}, 1 \mathrm{H}, \mathrm{ArH}), 5.94(\mathrm{~d}, J=$ $1.1 \mathrm{~Hz}, 1 \mathrm{H}, \mathrm{ArCH}), 4.26\left(\mathrm{ddd}, J=5.0,4.3,1.2 \mathrm{~Hz}, 2 \mathrm{H}, \mathrm{OCH}_{2}\right), 4.03-3.91\left(\mathrm{~m}, 2 \mathrm{H}, \mathrm{OCH}_{2}\right), 2.34-2.11$ $\left(\mathrm{m}, 1 \mathrm{H}, \mathrm{CH}_{2} \mathrm{CH}_{\mathrm{A}} \mathrm{CH}_{\mathrm{B}} \mathrm{CH}_{2}\right), 1.49-1.38\left(\mathrm{~m}, 1 \mathrm{H}, \mathrm{CH}_{2} \mathrm{CH}_{\mathrm{A}} \mathrm{CH}_{\mathrm{B}} \mathrm{CH}_{2}\right), 1.16(\mathrm{~s}, 21 \mathrm{H}, \mathrm{TIPS}) ;{ }^{13} \mathrm{C} \mathrm{NMR}(75 \mathrm{MHz}$, $\left.\mathrm{CDCl}_{3}\right) \delta \mathrm{ppm} 162.7\left(\mathrm{~d},{ }^{1} \mathrm{JC}-\mathrm{F}=249.9 \mathrm{~Hz}\right), 142.7\left(\mathrm{~d},{ }^{3} \mathrm{JC}-\mathrm{F}=7.9 \mathrm{~Hz}\right), 134.7\left(\mathrm{~d},{ }^{3} \mathrm{JC}-\mathrm{F}=8.2 \mathrm{~Hz}\right), 118.0(\mathrm{~d}$, $\left.{ }^{4} J \mathrm{C}-\mathrm{F}=3.4 \mathrm{~Hz}\right), 116.0\left(\mathrm{~d},{ }^{2} J \mathrm{C}-\mathrm{F}=22.2 \mathrm{~Hz}\right), 113.6\left(\mathrm{~d},{ }^{2} \mathrm{JC}-\mathrm{F}=23.7 \mathrm{~Hz}\right), 103.2,99.5\left(\mathrm{~d},{ }^{4} \mathrm{JC}-\mathrm{F}=1.4 \mathrm{~Hz}\right)$, 94.8, 67.8, 25.8, 18.8, 11.4; IR (thin film) $v_{\max }\left(\mathrm{cm}^{-1}\right): 2213.21(\mathrm{C} \equiv \mathrm{C})$; HRMS (ESI): m/z calculated for $\mathrm{C}_{19} \mathrm{H}_{31} \mathrm{O}_{2} \mathrm{FSi}: 363.2077$; for $[\mathrm{M}+\mathrm{H}]^{+}$found: 363.2184

Synthesis of ((2-(1,3-dioxan-2-yl)-4,5-dimethoxyphenyl)ethynyl)triisopropylsilane<smiles>COc1cc(C#C[Si](C(C)C)(C(C)C)C(C)C)c(C2OCCCO2)cc1OC</smiles>

General procedure B was followed using 4,5-dimethoxy-2-((triisopropylsilyl)ethynyl)benzaldehyde 6e $(2.460 \mathrm{~g}, 7.10 \mathrm{mmol})$. Concentrated in vacuo to give the title compound $(2.475 \mathrm{~g}, 86 \%)$ as an orange solid that was used in the subsequent step without further purification. mp 94-96 ${ }^{\circ} \mathrm{C} ;{ }^{1} \mathrm{H} \mathrm{NMR}\left(300 \mathrm{MHz}, \mathrm{CDCl}_{3}\right)$ $\delta$ ppm 7.16 (s, 1H, ArH), 6.90 (s, 1H, ArH), 5.95 (s, 1H, ArCH), 4.24 (dd, J = 11.3, 4.5 Hz, 2H, OCH $)$, $3.98\left(\mathrm{dd}, J=12.3,2.3 \mathrm{~Hz}, 2 \mathrm{H}, \mathrm{OCH}_{2}\right), 3.92\left(\mathrm{~s}, 3 \mathrm{H}, \mathrm{OCH}_{3}\right), 3.87\left(\mathrm{~s}, 3 \mathrm{H}, \mathrm{OCH}_{3}\right), 2.24(\mathrm{qt}, \mathrm{J}=12.6,5.0 \mathrm{~Hz}$, $\left.1 \mathrm{H}, \mathrm{CH}_{2} \mathrm{CH}_{\mathrm{A}} \mathrm{CH}_{\mathrm{B}} \mathrm{CH}_{2}\right), 1.49-1.37\left(\mathrm{~m}, 1 \mathrm{H}, \mathrm{CH}_{2} \mathrm{CH}_{\mathrm{A}} \mathrm{CH}_{\mathrm{B}} \mathrm{CH}_{2}\right), 1.16$ (s, 21H, TIPS); ${ }^{13} \mathrm{C}$ NMR (75 MHz, $\left.\mathrm{CDCl}_{3}\right) \delta$ ppm 149.8, 148.9, 134.0, 114.5, 114.2, 108.6, 104.3, 100.2, 93.3, 67.7, 56.1, 56.0, 25.8, 18.8, 11.5; IR (thin film) $v_{\max }\left(\mathrm{cm}^{-1}\right): 2141.94(\mathrm{C} \equiv \mathrm{C}), 1217.86$ and $1124.65\left(\mathrm{OCH}_{3}\right) ; \mathrm{HRMS}(\mathrm{ESI}): \mathrm{m} / \mathrm{z}$ calculated for $\mathrm{C}_{23} \mathrm{H}_{37} \mathrm{O}_{4} \mathrm{Si}: 405.2406$ for $[\mathrm{M}+\mathrm{H}]^{+}$; found: 405.2485 
Synthesis of ((2-(1,3-dioxan-2-yl)-4-methoxyphenyl)ethynyl)triisopropylsilane

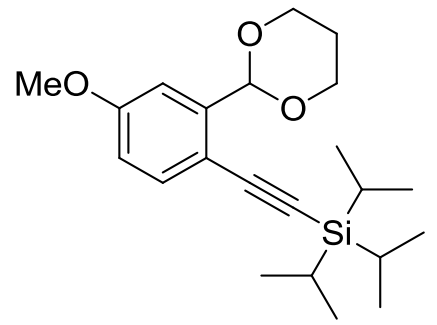

General procedure B was followed using 5-methoxy-2-((triisopropylsilyl)ethynyl)benzaldehyde $6 \mathbf{f}$ (2.629 $\mathrm{g}, 8.31 \mathrm{mmol})$. Concentrated in vacuo to give the title compound $(3.11 \mathrm{~g}, 99 \%)$ as a yellow solid that was used in the subsequent step without further purification. mp 58-59 ${ }^{\circ} \mathrm{C} ;{ }^{1} \mathrm{H} \mathrm{NMR}\left(300 \mathrm{MHz}, \mathrm{CDCl}_{3}\right) \delta \mathrm{ppm}$ $7.40(\mathrm{~d}, J=8.5 \mathrm{~Hz}, 1 \mathrm{H}, \operatorname{ArH}), 7.21(\mathrm{~d}, J=2.7 \mathrm{~Hz}, 1 \mathrm{H}, \operatorname{ArH}), 6.80(\mathrm{dd}, J=8.5,2.7 \mathrm{~Hz}, 1 \mathrm{H}, \operatorname{ArH}), 5.97$ (s, $1 \mathrm{H}, \mathrm{ArCH}), 4.24\left(\mathrm{dd}, J=11.0,5.0 \mathrm{~Hz}, 2 \mathrm{H}, \mathrm{OCH}_{2}\right), 3.96\left(\mathrm{td}, J=12.3,2.2 \mathrm{~Hz}, 2 \mathrm{H}, \mathrm{OCH}_{2}\right), 3.80(\mathrm{~s}, 3 \mathrm{H}$, $\left.\mathrm{OCH}_{3}\right), 2.31-2.09\left(\mathrm{~m}, 1 \mathrm{H}, \mathrm{CH}_{2} \mathrm{CH}_{\mathrm{A}} \mathrm{CH}_{\mathrm{B}} \mathrm{CH}_{2}\right), 1.41$ (d, J = $\left.13.4 \mathrm{~Hz}, 1 \mathrm{H}, \mathrm{CH}_{2} \mathrm{CH}_{\mathrm{A}} \mathrm{CH}_{\mathrm{B}} \mathrm{CH}_{2}\right), 1.16(\mathrm{~m}$, 21H, TIPS). ${ }^{13} \mathrm{C}$ NMR (75 MHz, $\left.\mathrm{CDCl}_{3}\right) \delta \mathrm{ppm} 159.9,141.8,134.0,115.2,114.1,110.6,104.4,100.0,92.9$, 67.6, 55.3, 25.8, 18.8, 11.4; IR(thin film) $v_{\max }\left(\mathrm{cm}^{-1}\right): 2145.60(\mathrm{C} \equiv \mathrm{C}), 1296.60$ and 1087.38 $\left(\mathrm{OCH}_{3}\right)$; HRMS (ESI): $\mathrm{m} / \mathrm{z}$ calculated for $\mathrm{C}_{22} \mathrm{H}_{34} \mathrm{O}_{3} \mathrm{Si}$ : 375.2277 ; for $[\mathrm{M}+\mathrm{H}]^{+}$found: 375.2381 ; for $[\mathrm{M}+\mathrm{Na}]^{+}$found: 397.2172

\section{Synthesis of (2-(2-(1,3-dioxan-2-yl)-3-fluorophenyl)ethynyl)triisopropylsilane}

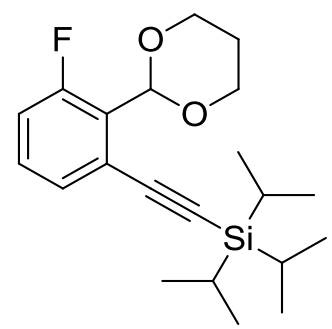

General procedure B was followed using 2-fluoro-6-(2-(triisopropylsilyl)ethynyl)benzaldehyde $\mathbf{6 g}$ (2.013 $\mathrm{g}, 6.61 \mathrm{mmol})$. Concentrated in vacuo to give the title compound as a yellow crystalline solid $(2.207 \mathrm{~g}, 92 \%)$ that was used in the subsequent step without further purification. mp $47^{\circ} \mathrm{C} ;{ }^{1} \mathrm{H} \mathrm{NMR}\left(300 \mathrm{MHz}, \mathrm{CDCl}_{3}\right) \delta$ ppm 7.24 (dd, $J=7.7,1.5 \mathrm{~Hz}, 1 \mathrm{H}, \mathrm{ArH}), 7.19$ (td, $J=7.8,5.0 \mathrm{~Hz}, 1 \mathrm{H}, \mathrm{ArH}), 7.05-6.96(\mathrm{~m}, 1 \mathrm{H}, \mathrm{ArH})$, $6.17(\mathrm{~d}, J=0.5 \mathrm{~Hz}, 1 \mathrm{H}, \mathrm{ArCH}), 4.23\left(\mathrm{dd}, J=11.0,4.9 \mathrm{~Hz}, 2 \mathrm{H}, \mathrm{OCH}_{2}\right), 3.89(\mathrm{dd}, J=11.9,10.9 \mathrm{~Hz}, 2 \mathrm{H}$, $\mathrm{OCH}_{2}$ ), 2.28 (tdd, $\left.J=12.6,7.7,5.0 \mathrm{~Hz}, 1 \mathrm{H}, \mathrm{CH}_{2} \mathrm{CH}_{\mathrm{A}} \mathrm{CH}_{\mathrm{B}} \mathrm{CH}_{2}\right), 1.38\left(\mathrm{~d}, J=13.4 \mathrm{~Hz}, 1 \mathrm{H}, \mathrm{CH}_{2} \mathrm{CH}_{\mathrm{A}} \mathrm{CH}_{\mathrm{B}} \mathrm{CH}_{2}\right.$ ), $1.15(\mathrm{~s}, 21 \mathrm{H}, \mathrm{TIPS}) ;{ }^{13} \mathrm{C}$ NMR $\left(75 \mathrm{MHz}, \mathrm{CDCl}_{3}\right) \delta \mathrm{ppm} 160.5\left(\mathrm{~d},{ }^{1} J_{\mathrm{C}-\mathrm{F}}=253.4 \mathrm{~Hz}\right), 129.7\left(\mathrm{~d},{ }^{3} J_{\mathrm{C}-\mathrm{F}}=9.6\right.$ $\mathrm{Hz}), 128.4\left(\mathrm{~d},{ }^{4} J_{\mathrm{C}-\mathrm{F}}=3.4 \mathrm{~Hz}\right), 126.3\left(\mathrm{~d},{ }^{2} J_{\mathrm{C}-\mathrm{F}}=13.4 \mathrm{~Hz}\right), 123.7\left(\mathrm{~d},{ }^{3} J_{\mathrm{C}-\mathrm{F}}=5.8 \mathrm{~Hz}\right), 116.6\left(\mathrm{~d},{ }^{2} J_{\mathrm{C}-\mathrm{F}}=22.5\right.$ $\mathrm{Hz}), 103.0\left(\mathrm{~d},{ }^{4} J_{\mathrm{C}-\mathrm{F}}=3.8 \mathrm{~Hz}\right), 99.5,95.7,67.4,25.3,18.2,10.8$; IR (thin film) $v_{\max }\left(\mathrm{cm}^{-1}\right): 2150.93(\mathrm{C} \equiv \mathrm{C})$; HRMS (ESI): m/z calculated for $\mathrm{C}_{21} \mathrm{H}_{31} \mathrm{O}_{2} \mathrm{FSi}$ : 363.2077 for $[\mathrm{M}+\mathrm{H}]^{+}$; found: 363.2138 . 
Synthesis of 3-(2-(1,3-dioxan-2-yl)phenyl)propiolate<smiles>COC(=O)C#Cc1ccccc1C1OCCCO1</smiles>

General procedure $\mathrm{C}$ was followed using ((2-(1,3-dioxan-2-yl)phenyl)ethynyl)triisopropylsilane (4.370 g, $12.7 \mathrm{mmol}$ ). Purified using column chromatography (20\% EtOAc in pentane) to give the pure product as a yellow oil (2.437 g, 78\%); ${ }^{1} \mathrm{H}$ NMR $\left(300 \mathrm{MHz}, \mathrm{CDCl}_{3}\right) \delta \mathrm{ppm} 7.70(\mathrm{ddd}, J=6.3,3.5,2.9 \mathrm{~Hz}, 1 \mathrm{H}, \mathrm{ArH}) 7.48$ (td, $J=7.7,1.3 \mathrm{~Hz}, 1 \mathrm{H}, \mathrm{ArH}) 7.34(\mathrm{td}, J=7.6,1.3 \mathrm{~Hz}, 1 \mathrm{H}, \mathrm{ArH}) 5.84(\mathrm{~s}, 1 \mathrm{H}, \mathrm{ArCH}) 4.26$ (ddd, $J=11.9$, $\left.5.0,1.2 \mathrm{~Hz}, 2 \mathrm{H}, \mathrm{OCH}_{2}\right) 4.05$ (td, $\left.J=12.3,2.5 \mathrm{~Hz}, 2 \mathrm{H}, \mathrm{OCH}_{2}\right) 3.85$ (s, 3H, $\left.\mathrm{OCH}_{3}\right) 2.25$ (dddt, J=22.7, 17.6, $\left.12.5,5.1 \mathrm{~Hz}, 1 \mathrm{H}, \mathrm{CH}_{2} \mathrm{CH}_{\mathrm{A}} \mathrm{CH}_{\mathrm{B}} \mathrm{CH}_{2}\right) 1.45\left(\mathrm{dtt}, J=13.5,2.5,1.3 \mathrm{~Hz} 1 \mathrm{H}, \mathrm{CH}_{2} \mathrm{CH}_{\mathrm{A}} \mathrm{CH}_{\mathrm{B}} \mathrm{CH}_{2}\right) ;{ }^{13} \mathrm{C} \mathrm{NMR}$ $\left(75 \mathrm{MHz}, \mathrm{CDCl}_{3}\right) \delta \mathrm{ppm} 154.5,141.9,133.6,131.1,128.9,126.3,118.0,99.7,84.4,83.9,67.7,52.9,25.7$; IR (thin film) $v_{\max }(\mathrm{cm}-1)$ : $2221.48(\mathrm{C} \equiv \mathrm{C}), 1707.32(\mathrm{C}=\mathrm{O})$; HRMS (ESI): $\mathrm{m} / \mathrm{z}$ calculated for $\mathrm{C}_{14} \mathrm{H}_{14} \mathrm{O}_{4}$ : 246.0892; for $[\mathrm{M}+\mathrm{H}]^{+}$found: 246.0892

Synthesis of Methyl 3-(6-(1,3-dioxan-2-yl)benzo[d][1,3]dioxol-5-yl)propiolate<smiles>COC(=O)C#Cc1cc2c(cc1C1OCCCO1)OCO2</smiles>

General procedure $\mathrm{C}$ was followed using ((6-(1,3-dioxan-2-yl)benzo[d][1,3]dioxol-5yl)ethynyl)triisopropylsilane $(1.151 \mathrm{~g}, 2.96 \mathrm{mmol})$. Purification by column chromatography (20\% EtOAc in Hexane) on silica afforded the title compound as a colorless solid $(0.460 \mathrm{~g}, 54 \%)$. mp $130-132{ }^{\circ} \mathrm{C} ;{ }^{1} \mathrm{H}$ NMR $\left(300 \mathrm{MHz}, \mathrm{CDCl}_{3}\right) \delta$ ppm 7.15 (s, 1H, ArH), 6.94 (s, 1H, ArH), $5.98\left(\mathrm{~s}, 2 \mathrm{H}, \mathrm{OCH}_{2} \mathrm{O}\right), 5.77(\mathrm{~s}, 1 \mathrm{H}$, $\mathrm{ArCH}), 4.28-4.16\left(\mathrm{~m}, 2 \mathrm{H}, \mathrm{OCH}_{2}\right), 4.07-3.95\left(\mathrm{~m}, 2 \mathrm{H}, \mathrm{OCH}_{2}\right), 3.82\left(\mathrm{~s}, 3 \mathrm{H}, \mathrm{OCH}_{3}\right), 2.31-2.09(\mathrm{~m}, 1 \mathrm{H}$, $\left.\mathrm{CH}_{2} \mathrm{CH}_{\mathrm{A}} \mathrm{CH}_{\mathrm{B}} \mathrm{CH}_{2}\right), 1.46-1.36\left(\mathrm{~m}, 1 \mathrm{H}, \mathrm{CH}_{2} \mathrm{CH}_{\mathrm{A}} \mathrm{CH}_{\mathrm{B}} \mathrm{CH}_{2}\right) ;{ }^{13} \mathrm{C} \mathrm{NMR}\left(75 \mathrm{MHz}, \mathrm{CDCl}_{3}\right) \delta \mathrm{ppm} 154.5,150.4$, 147.8, 138.7, 112.3, 111.1, 107.2, 102.1, 99.4, 84.3, 83.6, 67.6, 52.8, 25.7; IR (thin film) $v_{\max }\left(\mathrm{cm}^{-1}\right)$ : $2212.80(\mathrm{C} \equiv \mathrm{C}), 1700.44(\mathrm{C}=\mathrm{O}), 1244.40$ and $1094.93\left(\mathrm{OCH}_{2}\right)$; HRMS (ESI): $\mathrm{m} / \mathrm{z}$ calculated for $\mathrm{C}_{15} \mathrm{H}_{14} \mathrm{O}_{6}$ : 291.0790; for $[\mathrm{M}+\mathrm{H}]^{+}$found: 291.0853 ; for $[\mathrm{M}+\mathrm{Na}]^{+}$found: 313.0685 
Synthesis of Methyl 3-(2-(1,3-dioxan-2-yl)-4-(trifluoromethyl)phenyl)propiolate<smiles>COC(=O)C#Cc1ccc(C(F)(F)F)cc1C1OCCCO1</smiles>

General procedure $\quad \mathrm{C}$ was followed using ((2-(1,3-dioxan-2-yl)-4(trifluoromethyl)phenyl)ethynyl)triisopropylsilane $(1.830 \mathrm{~g}, 4.45 \mathrm{mmol})$. Purification by flash column chromatography (10\% EtOAc in hexane) afforded the title compound as an orange solid $(0.912 \mathrm{~g}, 65 \%)$. mp 68-71 ${ }^{\circ} \mathrm{C} ;{ }^{1} \mathrm{H}$ NMR $\left(300 \mathrm{MHz}, \mathrm{CDCl}_{3}\right) \delta \mathrm{ppm} 7.99(\mathrm{~s}, 1 \mathrm{H}, \mathrm{ArH}), 7.68(\mathrm{~d}, J=8.1 \mathrm{~Hz}, 1 \mathrm{H}, \mathrm{ArH}), 7.60(\mathrm{dd}$, $J=8.1,1.4 \mathrm{~Hz}, 1 \mathrm{H}, \mathrm{ArH}), 5.85(\mathrm{~s}, 1 \mathrm{H}, \mathrm{ArCH}), 4.29$ (ddd, $\left.J=11.8,5.0,1.1 \mathrm{~Hz}, 2 \mathrm{H}, \mathrm{OCH}_{2}\right), 4.06$ (ddd, $J=12.3$, $3.8,2.5 \mathrm{~Hz}, 2 \mathrm{H}, \mathrm{OCH}_{2}$ ), 3.87 (s, $3 \mathrm{H}, \mathrm{OCH}_{3}$ ), 2.27 (dddt, $J=22.7,17.6,12.5,5.1,1 \mathrm{H}, \mathrm{CH}_{2} \mathrm{CH}_{\mathrm{A}} \mathrm{CH}_{\mathrm{B}} \mathrm{CH}_{2}$ ), 1.57-1.40 (m, $\left.1 \mathrm{H}, \mathrm{CH}_{2} \mathrm{CH}_{\mathrm{A}} \mathrm{CH}_{\mathrm{B}} \mathrm{CH}_{2}\right) ;{ }^{13} \mathrm{C}$ NMR $\left(75 \mathrm{MHz}, \mathrm{CDCl}_{3}\right) \delta \mathrm{ppm} 154.1,142.7,133.8,132.6\left(\mathrm{q},{ }^{2} J \mathrm{C}-\right.$ $\mathrm{F}=33.1 \mathrm{~Hz}), 125.7\left(\mathrm{q},{ }^{3} \mathrm{JC}-\mathrm{F}=3.7 \mathrm{~Hz}\right), 123.7\left(\mathrm{q},{ }^{3} \mathrm{JC}-\mathrm{F}=3.9 \mathrm{~Hz}\right), 123.6\left(\mathrm{q},{ }^{1} \mathrm{JC}-\mathrm{F}=270.7 \mathrm{~Hz}\right), 98.9,85.9$, 81.9, 67.8, 53.2, 25.6; IR (thin film) $v_{\max }\left(\mathrm{cm}^{-1}\right): 222.37(\mathrm{C} \equiv \mathrm{C}), 1710.66(\mathrm{C}=\mathrm{O}), 1710.87(\mathrm{C}=\mathrm{O}), 1286.66$, 1171.09 and $1075.10\left(\mathrm{CF}_{3}\right)$; HRMS (ESI): $\mathrm{m} / \mathrm{z}$ calculated for $\mathrm{C}_{15} \mathrm{H}_{13} \mathrm{~F}_{3} \mathrm{O}_{4}: 315.0766$; for $[\mathrm{M}+\mathrm{H}]^{+}$found: 315.0825; for $[\mathrm{M}+\mathrm{Na}]^{+}$found 337.0661 .

\section{Synthesis of Methyl 3-(2-(1,3-dioxan-2-yl)-4-fluorophenyl)propiolate}<smiles>COC(=O)C#Cc1ccc(F)cc1C1OCCCO1</smiles>

General procedure $\mathrm{C}$ was followed using ((2-(1,3-dioxan-2-yl)-4-fluorophenyl)ethynyl)triisopropylsilane (3.21 g, $8.85 \mathrm{mmol})$. Purification by column chromatography (20\% EtOAc in hexane) afforded the title compound as a colorless solid $(1.741 \mathrm{~g}, 74 \%)$. mp 52-55 ${ }^{\circ} \mathrm{C} ;{ }^{1} \mathrm{H} \mathrm{NMR}\left(300 \mathrm{MHz}, \mathrm{CDCl}_{3}\right) \delta \mathrm{ppm} 7.54$ (dd, $J=8.6,5.4 \mathrm{~Hz}, 1 \mathrm{H}, \mathrm{ArH}), 7.40$ (dd, $J=9.4,2.7 \mathrm{~Hz}, 1 \mathrm{H}, \mathrm{ArH}), 7.06-6.97$ (m, 1H, ArH), 5.77 (s, 1H, ArCH), 4.23 (ddd, $\left.J=11.9,5.0,1.1 \mathrm{~Hz}, 2 \mathrm{H}, \mathrm{OCH}_{2}\right), 4.07-3.95\left(\mathrm{~m}, 2 \mathrm{H}, \mathrm{OCH}_{2}\right), 3.81\left(\mathrm{~s}, 3 \mathrm{H}, \mathrm{OCH}_{3}\right), 2.30-2.09(\mathrm{~m}$, $\left.1 \mathrm{H}, \mathrm{CH}_{2} \mathrm{CH}_{\mathrm{A}} \mathrm{CH}_{\mathrm{B}} \mathrm{CH}_{2}\right), 1.55-1.38\left(\mathrm{~m}, 1 \mathrm{H}, \mathrm{CH}_{2} \mathrm{CH}_{\mathrm{A}} \mathrm{CH}_{\mathrm{B}} \mathrm{CH}_{2}\right) ;{ }^{13} \mathrm{C} \mathrm{NMR}\left(75 \mathrm{MHz}, \mathrm{CDCl}_{3}\right) \delta \mathrm{ppm} 164.1(\mathrm{~d}$, $\left.{ }^{1} J \mathrm{C}-\mathrm{F}=253.9 \mathrm{~Hz}\right), 154.5,144.9\left(\mathrm{~d},{ }^{3} \mathrm{JC}-\mathrm{F}=8.4 \mathrm{~Hz}\right), 135.7\left(\mathrm{~d},{ }^{3} \mathrm{JC}-\mathrm{F}=8.8 \mathrm{~Hz}\right), 116.4\left(\mathrm{~d},{ }^{2} J \mathrm{C}-\mathrm{F}=22.5 \mathrm{~Hz}\right)$, $114.3\left(\mathrm{~d},{ }^{2} J \mathrm{C}-\mathrm{F}=24.2 \mathrm{~Hz}\right), 114.0\left(\mathrm{~d},{ }^{4} J \mathrm{C}-\mathrm{F}=3.4 \mathrm{~Hz}\right), 98.9,84.3,83.0,67.7,53.0,25.7$; IR (thin film) $v_{\max }$ $\left(\mathrm{cm}^{-1}\right): 2224.86(\mathrm{C} \equiv \mathrm{C}), 1710.91(\mathrm{C}=\mathrm{O})$; HRMS $(\mathrm{ESI}): \mathrm{m} / \mathrm{z}$ calculated for $\mathrm{C}_{14} \mathrm{H}_{13} \mathrm{O}_{4} \mathrm{~F}: 265.0798$; for $[\mathrm{M}+\mathrm{H}]^{+}$ found: 265.0874 ; for $[\mathrm{M}+\mathrm{Na}]^{+}$found: 287.0697 
<smiles>COC(=O)C#Cc1cc(OC)c(OC)cc1C1OCCCO1</smiles>

General procedure $\mathrm{C}$ was followed using ((2-(1,3-dioxan-2-yl)-4,5-dimethoxyphenyl) ethynyl)triisopropylsilane $(2.470 \mathrm{~g}, 6.10 \mathrm{mmol})$. Purification by column chromatography $(30 \%$ EtOAc in Hexane) afforded the title compound as a pale yellow solid (1.069 g, 57\%). mp 89-95 ${ }^{\circ} \mathrm{C} ;{ }^{1} \mathrm{H}$ NMR (300 $\left.\mathrm{MHz}, \mathrm{CDCl}_{3}\right) \delta \mathrm{ppm} 7.14(\mathrm{~s}, 1 \mathrm{H}, \mathrm{ArH}), 6.96$ (s, 1H, ArH), 5.75 (s, 1H, ArCH), 4.19 (dd, J = 11.0, 5.1 Hz, $\left.2 \mathrm{H}, \mathrm{OCH}_{2}\right), 3.99\left(\mathrm{td}, J=12.0,1.9 \mathrm{~Hz}, 2 \mathrm{H}, \mathrm{OCH}_{2}\right), 3.88\left(\mathrm{~s}, 3 \mathrm{H}, \mathrm{OCH}_{3}\right), 3.80\left(\mathrm{~s}, 3 \mathrm{H}, \mathrm{OCH}_{3}\right), 3.77(\mathrm{~s}, 3 \mathrm{H}$, $\mathrm{OCH}_{3}$ ), 2.18 (tdd, $\left.J=12.6,7.5,5.3 \mathrm{~Hz}, 1 \mathrm{H}, \mathrm{CH}_{2} \mathrm{CH}_{\mathrm{A}} \mathrm{CH}_{\mathrm{B}} \mathrm{CH}_{2}\right), 1.39$ (d, $J=13.5 \mathrm{~Hz}, 1 \mathrm{H}$, $\left.\mathrm{CH}_{2} \mathrm{CH}_{\mathrm{A}} \mathrm{CH}_{\mathrm{B}} \mathrm{CH}_{2}\right) ;{ }^{13} \mathrm{C}$ NMR $(75 \mathrm{MHz}, \mathrm{CDCl} 3) \delta \mathrm{ppm} \mathrm{154.7,} \mathrm{151.7,} \mathrm{149.0,} \mathrm{136.6,} \mathrm{115.3,} \mathrm{109.7,} \mathrm{108.9,}$ 99.6, 84.7, 83.8, 67.7, 56.2, 56.1, 52.8, 25.7; IR(thin film) $v_{\max }\left(\mathrm{cm}^{-1}\right): 2209.16(\mathrm{C} \equiv \mathrm{C}), 1701.41(\mathrm{C}=\mathrm{O})$, 1239.47 and $1122.39\left(\mathrm{OCH}_{3}\right)$; HRMS (ESI): m/z calculated for $\mathrm{C}_{16} \mathrm{H}_{18} \mathrm{O}_{6}: 306.1090$; for $[\mathrm{M}+\mathrm{H}]^{+}$found: $307.1169 \mathrm{~m} / \mathrm{z}$ calculated for $\mathrm{C}_{16} \mathrm{H}_{17} \mathrm{O}_{6} \mathrm{Na}: 329.2890$ for $[\mathrm{M}+\mathrm{Na}]^{+}$; found 329.0996

Synthesis of Methyl 3-(2-(1,3-dioxan-2-yl)-4-methoxyphenyl)propiolate<smiles>COC(=O)C#Cc1ccc(OC)cc1C1OCCCO1</smiles>

General procedure C was followed using ((2-(1,3-dioxan-2-yl)-4-methoxyphenyl)ethynyl) triisopropylsilane (3.110 g, $8.30 \mathrm{mmol})$. Purification by flash column chromatography (20\% EtOAc in hexane) afforded the title compound as a pale yellow solid (1.209 g, 53\%). mp 97-99 ${ }^{\circ} \mathrm{C} ;{ }^{1} \mathrm{H}$ NMR (300 $\left.\mathrm{MHz}, \mathrm{CDCl}_{3}\right) \delta$ ppm $7.47(\mathrm{~d}, J=8.6 \mathrm{~Hz}, 1 \mathrm{H}, \mathrm{ArH}), 7.20(\mathrm{~d}, J=2.7 \mathrm{~Hz}, 1 \mathrm{H}, \mathrm{ArH}), 6.82(\mathrm{dd}, J=8.6,2.7$ $\mathrm{Hz}, 1 \mathrm{H}, \mathrm{ArH}), 5.79$ (s, 1H, ArCH), 4.23 (dd, J=11.3, $\left.4.6 \mathrm{~Hz}, 2 \mathrm{H}, \mathrm{OCH}_{2}\right), 4.02(\mathrm{td}, J=12.3,2.4 \mathrm{~Hz}, 2 \mathrm{H}$, $\left.\mathrm{OCH}_{2}\right), 3.81\left(\mathrm{~s}, 3 \mathrm{H}, \mathrm{OCH}_{3}\right), 3.80\left(\mathrm{~s}, 3 \mathrm{H}, \mathrm{OCH}_{3}\right), 2.34-2.10\left(\mathrm{~m}, 1 \mathrm{H}, \mathrm{CH}_{2} \mathrm{CH}_{\mathrm{A}} \mathrm{CH}_{\mathrm{B}} \mathrm{CH}_{2}\right), 1.49-1.37(\mathrm{~m}$, $\left.1 \mathrm{H}, \mathrm{CH}_{2} \mathrm{CH}_{\mathrm{A}} \mathrm{CH}_{\mathrm{B}} \mathrm{CH}_{2}\right) ;{ }^{13} \mathrm{C} \mathrm{NMR}\left(75 \mathrm{MHz}, \mathrm{CDCl}_{3}\right) \delta \mathrm{ppm} 161.8,154.6,144.1,135.2,115.4,111.2,109.7$, 99.4, 84.7, 83.8, 67.6, 55.5, 52.6, 25.6; IR (thin film) $v_{\max }\left(\mathrm{cm}^{-1}\right): 2207.09(\mathrm{C} \equiv \mathrm{C}), 1701.24(\mathrm{C}=\mathrm{O}), 1298.02$ and 1086.50 $\left(\mathrm{OCH}_{3}\right)$; HRMS (ESI): m/z calculated for $\mathrm{C}_{15} \mathrm{H}_{16} \mathrm{O}_{5}: 276.0998$; for $[\mathrm{M}+\mathrm{H}]^{+}$found: 277.1065; for $[\mathrm{M}+\mathrm{Na}]^{+}$found: 299.0898 
<smiles>COC(=O)C#Cc1cccc(F)c1C1OCCCO1</smiles>

General procedure $\mathrm{C}$ was followed using (2-(2-(1,3-dioxan-2-yl)-3-fluorophenyl)ethynyl)triisopropylsilane ( $2.20 \mathrm{~g}, 6.07 \mathrm{mmol})$. Purification by column chromatography (20\% EtOAc in Hexane) afforded the title compound as a white crystalline solid $(1.082 \mathrm{~g}, 67 \%)$. mp $72-74{ }^{\circ} \mathrm{C} ;{ }^{1} \mathrm{H}$ NMR $\left(300 \mathrm{MHz}, \mathrm{CDCl}_{3}\right) \delta \mathrm{ppm}$ $7.37(\mathrm{dd}, J=7.7,1.1 \mathrm{~Hz}, 1 \mathrm{H}, \mathrm{ArH}), 7.28(\mathrm{td}, J=8.0,5.3 \mathrm{~Hz}, 1 \mathrm{H}, \mathrm{ArH}), 7.18-7.08(\mathrm{~m}, 1 \mathrm{H}, \mathrm{ArH}), 5.97(\mathrm{~s}$, $1 \mathrm{H}, \mathrm{ArCH}), 4.32-4.19\left(\mathrm{~m}, 2 \mathrm{H}, \mathrm{OCH}_{2}\right), 3.94\left(\mathrm{td}, J=12.3,2.2 \mathrm{~Hz}, 2 \mathrm{H}, \mathrm{OCH}_{2}\right), 3.82\left(\mathrm{~s}, 3 \mathrm{H}, \mathrm{OCH}_{3}\right), 2.36$ (qt, $\left.J=12.5,4.9 \mathrm{~Hz}, 1 \mathrm{H}, \mathrm{CH}_{2} \mathrm{CH}_{\mathrm{A}} \mathrm{CH}_{\mathrm{B}} \mathrm{CH}_{2}\right), 1.40\left(\mathrm{ddd}, J=12.4,2.5,1.3 \mathrm{~Hz}, 1 \mathrm{H}, \mathrm{CH}_{2} \mathrm{CH}_{\mathrm{A}} \mathrm{CH}_{\mathrm{B}} \mathrm{CH}_{2}\right) .{ }^{13} \mathrm{C}$ $\operatorname{NMR}\left(75 \mathrm{MHz}, \mathrm{CDCl}_{3}\right) \delta 160.2\left(\mathrm{~d},{ }^{1} J_{\mathrm{C}-\mathrm{F}}=252.1 \mathrm{~Hz}\right), 154.3,130.6\left(\mathrm{~d},{ }^{4} J_{\mathrm{C}-\mathrm{F}}=2.8 \mathrm{~Hz}\right), 130.5\left(\mathrm{~d}, J_{\mathrm{C}-\mathrm{F}}=3.2\right.$ $\mathrm{Hz}), 128.4\left(\mathrm{~d},{ }^{2} J_{\mathrm{C}-\mathrm{F}}=13.6 \mathrm{~Hz}\right), 120.3\left(\mathrm{~d},{ }^{3} J_{\mathrm{C}-\mathrm{F}}=5.4 \mathrm{~Hz}\right), 118.9\left(\mathrm{~d},{ }^{2} J_{\mathrm{C}-\mathrm{F}}=22.7 \mathrm{~Hz}\right), 97.1\left(\mathrm{~d},{ }^{3} J_{\mathrm{C}-\mathrm{F}}=4.6\right.$ $\mathrm{Hz}), 84.3,83.5\left(\mathrm{~d},{ }^{4} J_{\mathrm{C}-\mathrm{F}}=4.2 \mathrm{~Hz}\right), 67.8,52.8,25.4$; IR (thin film) $v_{\max }\left(\mathrm{cm}^{-1}\right): 2218.87(\mathrm{C} \equiv \mathrm{C}), 1710.88$ $(\mathrm{C}=\mathrm{O})$; HRMS (ESI): m/z calculated for $\mathrm{C}_{14} \mathrm{H}_{13} \mathrm{O}_{4} \mathrm{~F}: 265.0798$; for $[\mathrm{M}+\mathrm{H}]^{+}$found: 265.0859 .

\section{Synthesis of methyl 3-(2-formylphenyl)propiolate 3a}<smiles>COC(=O)C#Cc1ccccc1C=O</smiles>

General procedure D was followed using Methyl 3-(2-(1,3-dioxan-2-yl)phenyl)propiolate (2.437 g, 9.94 mmol) affording the title compound $(1.57 \mathrm{~g}, 84 \%)$ as a yellow crystalline solid that was used in the subsequent step without further purification. $\mathrm{mp} 54-56{ }^{\circ} \mathrm{C} ;{ }^{1} \mathrm{H} \mathrm{NMR}(500 \mathrm{MHz}, \mathrm{CDCl} 3) \delta 10.36(\mathrm{~s}, 1 \mathrm{H}$, CHO), 7.86 (dd, $J=7.4,1.5 \mathrm{~Hz}, 1 \mathrm{H}, \mathrm{ArH}), 7.60$ (dd, $J=7.3,1.4 \mathrm{~Hz}, 1 \mathrm{H}, \mathrm{ArH}), 7.55$ (td, $J=7.5,1.6 \mathrm{~Hz}$, $1 \mathrm{H}, \mathrm{ArH}), 7.51$ (tdd, $J=7.6,1.4,0.6 \mathrm{~Hz}, 1 \mathrm{H}, \mathrm{ArH}), 3.78\left(\mathrm{~s}, 3 \mathrm{H}, \mathrm{OCH}_{3}\right) .{ }^{13} \mathrm{C} \mathrm{NMR}\left(126 \mathrm{MHz}, \mathrm{CDCl}_{3}\right) \delta$ 190.0, 153.7, 137.1, 134.4, 133.8, 130.9, 127.8, 122.2, 86.0, 81.4, 52.9. IR (thin film) $v_{\max }\left(\mathrm{cm}^{-1}\right): 2220.10$ $(\mathrm{C} \equiv \mathrm{C}), 1691.80(\mathrm{C}=\mathrm{O})$.

Synthesis of Methyl 3-(6-formylbenzo[d][1,3]dioxol-5-yl)propiolate 3b<smiles>COC(=O)C#Cc1cc2c(cc1C=O)OCO2</smiles> 
General procedure $\mathrm{D}$ was followed using methyl 3-(6-(1,3-dioxan-2-yl)benzo[d][1,3]dioxol-5yl)propiolate $(0.450 \mathrm{~g}, 1.55 \mathrm{mmol})$. Concentrated in vacuo to give the title compound as a pale yellow solid $(0.359 \mathrm{~g}, 99 \%)$ that was used in the subsequent step without further purification. mp $185-188^{\circ} \mathrm{C} ;{ }^{1} \mathrm{H}$ NMR (300 MHz, $\left.\mathrm{CDCl}_{3}\right) \delta \mathrm{ppm} 10.33$ (s, 1H, CHO), 7.38 (s, 1H, ArH), 7.06 (s, 1H, ArH), $6.12\left(\mathrm{~s}, 2 \mathrm{H}, \mathrm{OCH}_{2} \mathrm{O}\right.$ ), $3.85\left(\mathrm{~s}, 3 \mathrm{H}, \mathrm{OCH}_{3}\right) ;{ }^{13} \mathrm{C} \mathrm{NMR}\left(75 \mathrm{MHz}, \mathrm{CDCl}_{3}\right) \delta \mathrm{ppm} 188.8,154.0,152.4,150.7,134.5,118.9,113.2$, 106.8, 103.0, 85.4, 81.7, 53.1; IR (thin film) $v_{\max }\left(\mathrm{cm}^{-1}\right)$ : $2214.05(\mathrm{C} \equiv \mathrm{C}), 1707.20(\mathrm{C}=\mathrm{O}), 1679.52(\mathrm{C}=\mathrm{O})$, 1247.61 and $1032.77\left(\mathrm{OCH}_{2}\right)$; HRMS (ESI): $\mathrm{m} / \mathrm{z}$ calculated for $\mathrm{C}_{12} \mathrm{H}_{8} \mathrm{O}_{5}: 233.0372$; for $[\mathrm{M}+\mathrm{H}]^{+}$found: 233.0446; for $[\mathrm{M}+\mathrm{Na}]^{+}$found 255.0255 .

Synthesis of methyl 3-(2-formyl-4-(trifluoromethyl)phenyl)propiolate 3c<smiles>COC(=O)C#Cc1ccc(C(F)(F)F)cc1C=O</smiles>

General procedure D was followed using methyl 3-(2-(1,3-dioxan-2-yl)-4(trifluoromethyl)phenyl)propiolate $(0.912 \mathrm{~g}, 2.90 \mathrm{mmol})$. Concentrated in vacuo to give the title compound as a clear oil $(0.586 \mathrm{~g}, 79 \%)$ that was used in the subsequent step without further purification.; ${ }^{1} \mathrm{H}$ NMR (300MHz, $\left.\mathrm{CDCl}_{3}\right) \delta \mathrm{ppm} 10.43$ (s, 1H, CHO), 8.15 (s, 1H, ArH), 7.84 (dd, $\left.J=8.3,1.5,1 \mathrm{H}, \mathrm{ArH}\right), 7.80$ (d,

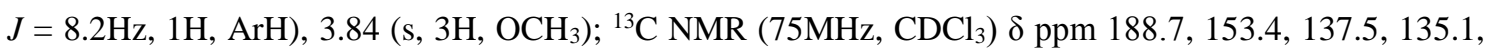
$132.9\left(\mathrm{q},{ }^{2} \mathrm{JC}-\mathrm{F}=33.9 \mathrm{~Hz}\right), 130.2\left(\mathrm{q},{ }^{3} \mathrm{JC}-\mathrm{F}=3.5 \mathrm{~Hz}\right), 125.8\left(\mathrm{q},{ }^{4} \mathrm{JC}-\mathrm{F}=1.0 \mathrm{~Hz}\right), 124.9\left(\mathrm{q},{ }^{3} J \mathrm{C}-\mathrm{F}=3.9 \mathrm{~Hz}\right)$, $123.0\left(\mathrm{q},{ }^{1} J \mathrm{C}-\mathrm{F}=272.9 \mathrm{~Hz}\right), 87.9,79.5,53.3$; IR (thin film) $v_{\max }\left(\mathrm{cm}^{-1}\right): 2229.81(\mathrm{C} \equiv \mathrm{C}), 1706.19(\mathrm{C}=\mathrm{O})$, 1283.75, 1169.69 and $1127.20\left(\mathrm{CF}_{3}\right)$; HRMS (ESI): $\mathrm{m} / \mathrm{z}$ calculated for $\mathrm{C}_{12} \mathrm{H}_{7} \mathrm{~F}_{3} \mathrm{O}_{3}: 257.04255$; for $[\mathrm{M}+\mathrm{H}]^{+}$ found 257.0415; for [M+Na] $]^{+}$found 279.0191 .

\section{Synthesis of methyl 3-(2-formyl-4-fluorophenyl)propiolate 3d}<smiles>COC(=O)C#Cc1ccc(F)cc1C=O</smiles>

General procedure D was followed using methyl 3-(2-(1,3-dioxan-2-yl)-4-fluorophenyl)propiolate (1.59 g, $6.00 \mathrm{mmol})$. Concentrated in vacuo to give the title compound $(1.02 \mathrm{~g}, 82 \%)$ as an orange solid that was used in the subsequent step without further purification. mp 61-63 ${ }^{\circ} \mathrm{C} ;{ }^{1} \mathrm{H} \mathrm{NMR}\left(300 \mathrm{MHz}, \mathrm{CDCl}_{3}\right) \delta \mathrm{ppm}$ $10.44(\mathrm{~d}, J=3.0 \mathrm{~Hz}, 1 \mathrm{H}, \mathrm{CHO}), 7.72(\mathrm{dd}, J=8.6,5.0 \mathrm{~Hz}, 1 \mathrm{H}, \mathrm{ArH}), 7.64(\mathrm{dd}, J=8.4,2.7 \mathrm{~Hz}, 1 \mathrm{H}, \mathrm{ArH})$, $7.33(\mathrm{ddd}, J=8.5,7.7,2.7 \mathrm{~Hz}, 1 \mathrm{H}, \mathrm{ArH}), 3.86\left(\mathrm{~s}, 3 \mathrm{H}, \mathrm{OCH}_{3}\right) ;{ }^{13} \mathrm{C} \mathrm{NMR}\left(75 \mathrm{MHz}, \mathrm{CDCl}_{3}\right) \delta \mathrm{ppm} 189.1$ (d, $\left.{ }^{4} J \mathrm{C}-\mathrm{F}=1.5 \mathrm{~Hz}\right), 164.0\left(\mathrm{~d},{ }^{1} J \mathrm{C}-\mathrm{F}=256.6 \mathrm{~Hz}\right), 153.8,139.8\left(\mathrm{~d},{ }^{3} J \mathrm{C}-\mathrm{F}=7.1 \mathrm{~Hz}\right), 136.9\left(\mathrm{~d},{ }^{3} J \mathrm{C}-\mathrm{F}=8.2 \mathrm{~Hz}\right)$, $121.6\left(\mathrm{~d},{ }^{2} \mathrm{JC}-\mathrm{F}=22.9 \mathrm{~Hz}\right), 118.7\left(\mathrm{~d},{ }^{4} \mathrm{JC}-\mathrm{F}=3.6 \mathrm{~Hz}\right), 114.7\left(\mathrm{~d},{ }^{2} \mathrm{JC}-\mathrm{F}=23.3 \mathrm{~Hz}\right), 86.2\left(\mathrm{~d},{ }^{5} \mathrm{JC}-\mathrm{F}=1.8 \mathrm{~Hz}\right)$ 
80.5, 53.2; IR (thin film) $v_{\max }\left(\mathrm{cm}^{-1}\right): 2226.50(\mathrm{C} \equiv \mathrm{C}), 1712.63(\mathrm{C}=\mathrm{O}), 1696.53(\mathrm{C}=\mathrm{O})$; HRMS (ESI): $\mathrm{m} / \mathrm{z}$ calculated for $\mathrm{C}_{11} \mathrm{H}_{7} \mathrm{FO}_{3}: 207.0379$; for $[\mathrm{M}+\mathrm{H}]^{+}$found: 207.0455 ; for $[\mathrm{M}+\mathrm{Na}]^{+}$found: 229.0252

Synthesis of methyl 3-(2-formyl-4,5-dimethoxyphenyl)propiolate 3e<smiles>COC(=O)C#Cc1cc(OC)c(OC)cc1C=O</smiles>

General procedure D was followed using methyl 3-(2-(1,3-dioxan-2-yl)-4,5-dimethoxyphenyl)propiolate $(1.069 \mathrm{~g}, 3.49 \mathrm{mmol})$. Concentrated in vacuo to give the title compound $(0.721 \mathrm{~g}, 83 \%)$ as a pale yellow solid that was used in the subsequent step without further purification. mp $107-111^{\circ} \mathrm{C} ;{ }^{1} \mathrm{H} \mathrm{NMR}(300 \mathrm{MHz}$, $\left.\mathrm{CDCl}_{3}\right) \delta \mathrm{ppm} 10.36$ (s, 1H, CHO), 7.44 (s, 1H, ArH), 7.11 (s, 1H, ArH), 3.97 (s, 6H), 3.86 (s, 3H, $\mathrm{OCH}_{3}$ ); ${ }^{13} \mathrm{C}$ NMR $\left(75 \mathrm{MHz}, \mathrm{CDCl}_{3}\right) \delta \mathrm{ppm} 189.2,154.1,153.5,151.6,132.2,116.8,115.5,108.6,85.3,82.0,56.5$, 56.4, 53.1; IR(thin film) $v_{\max }\left(\mathrm{cm}^{-1}\right): 2218.72(\mathrm{C} \equiv \mathrm{C}), 1715.47(\mathrm{C}=\mathrm{O}), 1692.76(\mathrm{C}=\mathrm{O}), 1246.12$ and 1104.53 $\left(\mathrm{OCH}_{3}\right)$; HRMS (ESI): $\mathrm{m} / \mathrm{z}$ calculated for $\mathrm{C}_{13} \mathrm{H}_{12} \mathrm{O}_{5}: 249.0685$; for $[\mathrm{M}+\mathrm{H}]^{+}$found: 249.0756

\section{Synthesis of Methyl 3-(2-formyl-4-methoxyphenyl)propiolate 3f}<smiles>COC(=O)C#Cc1ccc(OC)cc1C=O</smiles>

General procedure D was followed using methyl 3-(2-(1,3-dioxan-2-yl)-4-methoxyphenyl)propiolate 36.2 $(1.191 \mathrm{~g}, 4.31 \mathrm{mmol})$. Concentrated in vacuo to give the title compound $(0.906 \mathrm{~g}, 96 \%)$ as a yellow solid that was used in the subsequent step without further purification. mp 68-70 ${ }^{\circ} \mathrm{C} ;{ }^{1} \mathrm{H} \mathrm{NMR}\left(300 \mathrm{MHz}, \mathrm{CDCl}_{3}\right)$ $\delta$ ppm 10.42 (s, $1 \mathrm{H}, \mathrm{CHO}), 7.60(\mathrm{~d}, J=8.6 \mathrm{~Hz}, 1 \mathrm{H}, \mathrm{ArH}), 7.41(\mathrm{~d}, J=2.7 \mathrm{~Hz}, 1 \mathrm{H}, \mathrm{ArH}), 7.12(\mathrm{dd}, J=8.6$, $2.8 \mathrm{~Hz}, 1 \mathrm{H}, \mathrm{ArH}), 3.87\left(\mathrm{~s}, 3 \mathrm{H}, \mathrm{OCH}_{3}\right), 3.83\left(\mathrm{~s}, 3 \mathrm{H}, \mathrm{OCH}_{3}\right) ;{ }^{13} \mathrm{C} \mathrm{NMR}\left(75 \mathrm{MHz}, \mathrm{CDCl}_{3}\right) \delta \mathrm{ppm} 190.3,161.7$, 154.2, 139.1, 136.2, 121.3, 114.7, 111.0, 85.4, 82.2, 55.9, 53.0; IR (thin film) $v_{\max }\left(\mathrm{cm}^{-1}\right): 2213.52(\mathrm{C} \equiv \mathrm{C})$, 1711.31(C=O), $1695.48(\mathrm{C}=\mathrm{O}), 1286.52$ and $1087.51\left(\mathrm{OCH}_{3}\right)$; HRMS (ESI): $\mathrm{m} / \mathrm{z}$ calculated for $\mathrm{C}_{12} \mathrm{H}_{10} \mathrm{O}_{4}$ : 219.0579; for $[\mathrm{M}+\mathrm{H}]^{+}$found: 219.0656; for $[\mathrm{M}+\mathrm{Na}]^{+}$found 241.0475 .

\section{Synthesis of methyl 3-(3-fluoro-2-formylphenyl)propiolate 3g}<smiles>COC(=O)C#Cc1cccc(F)c1C=O</smiles> 
General procedure D was followed using methyl 3-(2-(1,3-dioxan-2-yl)-3-fluorophenyl)propiolate (1.052 $\mathrm{g}, 3.98 \mathrm{mmol})$. Concentrated in vacuo to give the title compound $(0.747 \mathrm{~g}, 91 \%)$ as a pale yellow solid that was used in the subsequent step without further purification. mp 66-68 ${ }^{\circ} \mathrm{C} ;{ }^{1} \mathrm{H} \mathrm{NMR}\left(500 \mathrm{MHz}, \mathrm{CDCl}_{3}\right) \delta$ ppm 10.44 (s, 1H, CHO), 7.60 (td, $J=8.0,5.5 \mathrm{~Hz}, 1 \mathrm{H}, \mathrm{ArH}), 7.49$ (d, $J=7.7 \mathrm{~Hz}, 1 \mathrm{H}, \mathrm{ArH}$ ), $7.31-7.25$ $(\mathrm{m}, 1 \mathrm{H}, \mathrm{ArH}), 3.85\left(\mathrm{~s}, 3 \mathrm{H}, \mathrm{OCH}_{3}\right) ;{ }^{13} \mathrm{C}$ NMR $\left(126 \mathrm{MHz}, \mathrm{CDCl}_{3}\right) \delta \mathrm{ppm} 186.3\left(\mathrm{~d},{ }^{3} J_{\mathrm{C}-\mathrm{F}}=4.8 \mathrm{~Hz}\right), 163.2(\mathrm{~d}$, $\left.{ }^{1} J_{\mathrm{C}-\mathrm{F}}=262.3 \mathrm{~Hz}\right), 153.6,135.2\left(\mathrm{~d},{ }^{3} J_{\mathrm{C}-\mathrm{F}}=10.4 \mathrm{~Hz}\right), 131.1\left(\mathrm{~d},{ }^{4} J_{\mathrm{C}-\mathrm{F}}=3.6 \mathrm{~Hz}\right), 125.2\left(\mathrm{~d},{ }^{2} J_{\mathrm{C}-\mathrm{F}}=8.7 \mathrm{~Hz}\right)$, $122.0\left(\mathrm{~d},{ }^{3} J_{\mathrm{C}-\mathrm{F}}=3.5 \mathrm{~Hz}\right), 119.2\left(\mathrm{~d},{ }^{2} J_{\mathrm{C}-\mathrm{F}}=21.4 \mathrm{~Hz}\right), 85.7,81.6\left(\mathrm{~d},{ }^{4} J_{\mathrm{C}-\mathrm{F}}=4.4 \mathrm{~Hz}\right), 53.0$; IR (thin film) $v_{\max }$ $\left(\mathrm{cm}^{-1}\right): 2218.87(\mathrm{C} \equiv \mathrm{C}), 1708.24(\mathrm{C}=\mathrm{O}), 1693.47(\mathrm{C}=\mathrm{O})$; HRMS $(\mathrm{ESI}): \mathrm{m} / \mathrm{z}$ calculated for $\mathrm{C}_{11} \mathrm{H}_{7} \mathrm{O}_{3} \mathrm{~F}$ : 207.0379; for $[\mathrm{M}+\mathrm{H}]^{+}$found: 207.0412 .

\section{Synthesis of 3-(2-(1,3-dioxan-2-yl)-4-(trifluoromethyl)phenyl)propiolic acid}<smiles>O=C(O)C#Cc1ccc(C(F)(F)F)cc1C1OCCCO1</smiles>

To a stirred solution of Methyl 3-(2-(1,3-dioxan-2-yl)-4-(trifluoromethyl)phenyl)propiolate (0.68 g, 2.16 mmol, 1 eq) in $\mathrm{EtOH}(17 \mathrm{~mL})$ was added $\mathrm{NaOH}$ solution $(0.2 \mathrm{M}, 10.82 \mathrm{~mL}, 1.1 \mathrm{eq})$ at $0{ }^{\circ} \mathrm{C}$, the reaction was then allowed to warm to $\mathrm{rt}$ and stirred for $2 \mathrm{~h}$. The reaction was then diluted with $\mathrm{H}_{2} \mathrm{O}(20 \mathrm{~mL})$ and washed with DCM $(2 \times 20 \mathrm{~mL})$ the aqueous layer is then acidifed with $3 \mathrm{M} \mathrm{HCl}$ until the solution reaches $\mathrm{pH}$ of 1 . The aqueous layer is then extracted with EtOAc $(3 \times 20 \mathrm{~mL})$. The combined organic layers were then dried $\mathrm{MgSO}_{4}$ and concentrated in vacuo to give the title compound as a white solid $(0.573 \mathrm{~g}, 87 \%)$ which was used in the next step without further purification. mp $140-144{ }^{\circ} \mathrm{C} ;{ }^{1} \mathrm{H}$ NMR $\left(300 \mathrm{MHz}, \mathrm{CDCl}_{3}\right)$ $\delta=10.16$ (br. s., $1 \mathrm{H}, \mathrm{OH}$ ), 8.02 (s, $1 \mathrm{H}, \mathrm{ArH}), 7.72$ (d, $J=8.3 \mathrm{~Hz}, 1 \mathrm{H}, \mathrm{ArH}), 7.63$ (dd, $J=1.3,8.1 \mathrm{~Hz}, 1$ $\mathrm{H}, \mathrm{ArH}), 5.87\left(\mathrm{~s}, 1 \mathrm{H}, \mathrm{CH}(\mathrm{O})_{2}\right), 4.40-4.23\left(\mathrm{~m}, 2 \mathrm{H}, \mathrm{CH}_{2} \mathrm{CH}_{\mathrm{A}} \mathrm{CH}_{\mathrm{B}} \mathrm{CH}_{2}\right), 4.08(\mathrm{dt}, J=2.6,12.2 \mathrm{~Hz}, 2 \mathrm{H}$, $\left.\mathrm{CH}_{2} \mathrm{CH}_{\mathrm{A}} \mathrm{CH}_{\mathrm{B}} \mathrm{CH}_{2}\right), 2.42$ - 2.19 (m, $\left.1 \mathrm{H}, \mathrm{CH}_{2} \mathrm{CH}_{\mathrm{A}} \mathrm{CH}_{\mathrm{B}} \mathrm{CH}_{2}\right), 1.59-1.42\left(\mathrm{~m}, 1 \mathrm{H}, \mathrm{CH}_{2} \mathrm{CH}_{\mathrm{A}} \mathrm{CH}_{\mathrm{B}} \mathrm{CH}_{2}\right) ;{ }^{13} \mathrm{C}$ $\operatorname{NMR}\left(75 \mathrm{MHz}, \mathrm{CDCl}_{3}\right) \delta=157.6,142.7,133.9,132.8\left(\mathrm{q},{ }^{2} \mathrm{C}-\mathrm{F}=33.9 \mathrm{~Hz}\right), 125.7\left(\mathrm{q},{ }^{3} J \mathrm{C}-\mathrm{F}=3.8 \mathrm{~Hz}\right)$, $123.7\left(\mathrm{q},{ }^{3} \mathrm{JC}-\mathrm{F}=3.8 \mathrm{~Hz}\right), 121.2\left(\mathrm{app} \mathrm{d},{ }^{3} J \mathrm{C}-\mathrm{F}=1.5 \mathrm{~Hz}\right), 98.7,85.4,84.1,67.6$; IR (thin film) $v_{\max }\left(\mathrm{cm}^{-1}\right)$ : $2869.53(\mathrm{OH}), 2239.37(\mathrm{C} \equiv \mathrm{C}), 1679.92\left(\mathrm{C}=\mathrm{O}\right.$ br); $\mathrm{HRMS}(\mathrm{ESI}): \mathrm{m} / \mathrm{z}$ calculated for $\mathrm{C}_{14} \mathrm{H}_{11} \mathrm{~F}_{3} \mathrm{O}_{4}: 301.06094$; for $[\mathrm{M}+\mathrm{H}]^{+}$found: 301.0623 ; for $[\mathrm{M}+\mathrm{Na}]^{+}$found 323.0450 .

\section{Synthesis of 3-(2-(1,3-dioxan-2-yl)-4-(trifluoromethyl)phenyl)-N-phenylpropiolamide}<smiles>O=C(C#Cc1ccc(C(F)(F)F)cc1C1OCCCO1)Nc1ccccc1</smiles> 
To a stirres solution of 3-(2-(1,3-dioxan-2-yl)-4-(trifluoromethyl)phenyl)propiolic acid (0.573 g, $1.9 \mathrm{mmol}$, $1 \mathrm{eq})$ in DCM $(10 \mathrm{~mL})$ at $-20{ }^{\circ} \mathrm{C}$ was added a solution of DMAP (23 mg, $\left.0.19 \mathrm{mmol}, 0.1 \mathrm{eq}\right)$ and DCC $(0.431 \mathrm{~g}, 2.09 \mathrm{mmol}, 1.1 \mathrm{eq})$ in DCM $(10 \mathrm{~mL})$. Aniline $(0.175 \mathrm{~mL}, 1.9 \mathrm{mmol}, 1 \mathrm{eq})$ was then added dropwise, the reaction was allowed to warm to $\mathrm{rt}$ and stirred for $12 \mathrm{~h}$. The reaction was filtered through Celite ${ }^{\circ}$, the mother liquor is then further diluted with DCM $(50 \mathrm{~mL})$ and the combined organic layers were washed with $0.5 \mathrm{M} \mathrm{HCl}(3 \times 50 \mathrm{~mL})$, the organic layers were then dried $\mathrm{MgSO}_{4}$ and concentrated in vacuo purification by column chromatography (20\% EtOAc in pentane) afforded the title compound as a white solid ( $0.5 \mathrm{~g}, 70 \%$ ). mp 76-77 ${ }^{\circ} \mathrm{C} ;{ }^{1} \mathrm{H}$ NMR (300MHz, $\left.\mathrm{CDCl}_{3}\right) \delta=8.02$ (s, $\left.1 \mathrm{H}, \mathrm{ArH}\right), 7.74$ (br. s., $\left.1 \mathrm{H}, \mathrm{NH}\right)$, 7.68 - 7.53 (m, 4 H, ArH), 7.38 (t, J=7.9 Hz, 2 H, ArH), 7.24 - 7.11 (m, 1 H, ArH), 5.90 (s, 1 H, CH(O) $)_{2}$ ), $4.31\left(\mathrm{dd}, J=5.1,10.7 \mathrm{~Hz}, 2 \mathrm{H}, \mathrm{CH}_{2} \mathrm{CH}_{\mathrm{A}} \mathrm{CH}_{\mathrm{B}} \mathrm{CH}_{2}\right.$ ), 4.09 (dt, $J=2.3,12.2 \mathrm{~Hz}, 2 \mathrm{H}, \mathrm{CH}_{2} \mathrm{CH}_{\mathrm{A}} \mathrm{CH}_{\mathrm{B}} \mathrm{CH}_{2}$ ), 2.37 - $2.18\left(\mathrm{~m}, 1 \mathrm{H}, \mathrm{CH}_{2} \mathrm{CH}_{\mathrm{A}} \mathrm{CH}_{\mathrm{B}} \mathrm{CH}_{2}\right), 1.56-1.42\left(\mathrm{~m}, 1 \mathrm{H}, \mathrm{CH}_{2} \mathrm{CH}_{\mathrm{A}} \mathrm{CH}_{\mathrm{B}} \mathrm{CH}_{2}\right) ;{ }^{13} \mathrm{C} \mathrm{NMR}\left(75 \mathrm{MHz}, \mathrm{CDCl}_{3}\right) \delta=$ 150.2, 142.1, 137.1, 133.1, 132.1 (app d, $\left.{ }^{2} \mathrm{JC}-\mathrm{F}=42.3 \mathrm{~Hz}\right), 129.2,125.6$ (q, $\left.{ }^{3} \mathrm{JC}-\mathrm{F}=3.0 \mathrm{~Hz}\right), 125.2,123.7$ $\left(\mathrm{q},{ }^{3} \mathrm{JC}-\mathrm{F}=3.8 \mathrm{~Hz}\right), 122.0\left(\mathrm{app} \mathrm{d},{ }^{3} \mathrm{JC}-\mathrm{F}=0.76 \mathrm{~Hz}\right), 119.9,98.8,88.7,81.4,77.2,67.6,25.5$; IR (thin film) $v_{\max }\left(\mathrm{cm}^{-1}\right): 3262.69(\mathrm{NH}), 2212.32(\mathrm{C} \equiv \mathrm{C}), 1637.61$ and $1597.89(\mathrm{C}=\mathrm{O})$; HRMS (ESI): m/z calculated for $\mathrm{C}_{20} \mathrm{H}_{16} \mathrm{~F}_{3} \mathrm{~N}_{1} \mathrm{O}_{3}$ : 376.116053 ; for $[\mathrm{M}+\mathrm{H}]^{+}$found: 376.1163 ; for $[\mathrm{M}+\mathrm{Na}]^{+}$found 398.0990 .

\section{Synthesis of 3-(2-formyl-4-(trifluoromethyl)phenyl)-N-phenylpropiolamide 8}<smiles>O=Cc1cc(C(F)(F)F)ccc1C#CC(=O)Nc1ccccc1</smiles>

General procedure D was followed using 3-(2-(1,3-dioxan-2-yl)-4-(trifluoromethyl)phenyl)-Nphenylpropiolamide $(0.36 \mathrm{~g}, .96 \mathrm{mmol})$. Concentrated in vacuo to give the title compound $(0.292 \mathrm{~g}, 96 \%)$ as a pale yellow solid that was used in the subsequent step without further purification. mp $149-151^{\circ} \mathrm{C}$ (decomp); ${ }^{1} \mathrm{H}$ NMR $\left(300 \mathrm{MHz}, \mathrm{CDCl}_{3}\right) \delta=10.50$ (s, $\left.1 \mathrm{H}, \mathrm{C}(\mathrm{O}) \mathrm{H}\right), 8.20(\mathrm{~s}, 1 \mathrm{H}, \mathrm{NH}), 8.16-8.04$ (m, $1 \mathrm{H}$, ArH), 7.90 - 7.78 (m, 2 H, ArH), 7.64 - 7.54 (m, 2 H, ArH), 7.37 (t, J= 7.9 Hz, 3 H, ArH), 7.23 - 7.13 (m, $1 \mathrm{H}, \mathrm{ArH}) ;{ }^{13} \mathrm{C} \mathrm{NMR}\left(75 \mathrm{MHz}, \mathrm{CDCl}_{3}\right) \delta=189.1,149.7,137.1,136.9,134.9,132.8,130.9\left(\mathrm{q},{ }^{3} \mathrm{JC}-\mathrm{F}=3.8\right.$ $\mathrm{Hz}), 130.2$, 129.2, 125.8, 125.7 (app d, $\left.{ }^{3} J \mathrm{C}-\mathrm{F}=3.8 \mathrm{~Hz}\right), 125.4,120.1,90.8,79.5$; IR (thin film) $v_{\max }\left(\mathrm{cm}^{-}\right.$ $\left.{ }^{1}\right): 3342.82(\mathrm{NH}), 222.08(\mathrm{C} \equiv \mathrm{C}), 1688.62$ and $1645.77(\mathrm{C}=\mathrm{O}) ; \mathrm{HRMS}(\mathrm{ESI}): \mathrm{m} / \mathrm{z}$ calculated for $\mathrm{C}_{17} \mathrm{H}_{10} \mathrm{~F}_{3} \mathrm{~N}_{1} \mathrm{O}_{2}: 318.07418$; for $[\mathrm{M}+\mathrm{H}]^{+}$found: 318.0715 ; for $[\mathrm{M}+\mathrm{Na}]^{+}$found 340.0532 .

\section{Synthesis of methyl 2-(2-hydroxy-3-oxoisoindolin-1-yl)acetate 4a}<smiles>COC(=O)CC1c2ccccc2C(=O)N1O</smiles> 
General procedure E was followed using methyl 3-(2-formylphenyl)propiolate (1.570 g, 8.31 mmol) Purified by column chromatography (5\% MeOH in DCM) to give the title compound as a white solid (1.414 g, 70\%). mp 137-139 ${ }^{\circ} \mathrm{C} ;{ }^{1} \mathrm{H}$ NMR $\left(500 \mathrm{MHz}, \mathrm{CDCl}_{3}\right) \delta \mathrm{ppm} 7.74(\mathrm{~d}, J=7.7 \mathrm{~Hz}, 1 \mathrm{H}, \mathrm{ArH}), 7.53(\mathrm{t}, J=7.5$ $\mathrm{Hz}, 1 \mathrm{H}, \mathrm{ArH}), 7.48-7.42$ (m, 2H, ArH), 5.13 (dd, J=8.4, 4.6 Hz, 1H, ArCHN), 3.76 (s, 3H, OCH $)_{3}, 3.31$ $\left(\mathrm{dd}, J=16.4,4.6 \mathrm{~Hz}, 1 \mathrm{H}, \mathrm{CH}_{\mathrm{A}} \mathrm{CH}_{\mathrm{B}}\right), 2.65\left(\mathrm{dd}, J=16.4,8.5 \mathrm{~Hz}, 1 \mathrm{H}, \mathrm{CH}_{\mathrm{A}} \mathrm{CH}_{\mathrm{B}}\right) ;{ }^{13} \mathrm{C} \mathrm{NMR}\left(75 \mathrm{MHz}, \mathrm{CDCl}_{3}\right)$ $\delta$ ppm 171.0, 164.9, 141.8, 132.2, 129.7, 128.8, 123.5, 122.7, 59.1, 52.2, 35.9; IR (thin film) $v_{\max }\left(\mathrm{cm}^{-1}\right):$ br peak at $3077.60(\mathrm{O}-\mathrm{H}), 1734.01(\mathrm{C}=\mathrm{O}), 1699.60(\mathrm{C}=\mathrm{O})$; HRMS $(\mathrm{ESI}): \mathrm{m} / \mathrm{z}$ calculated for $\mathrm{C}_{11} \mathrm{H}_{11} \mathrm{NO}_{4}$ : 222.0688; for $[\mathrm{M}+\mathrm{H}]^{+}$found: 222.0763 ; for $[\mathrm{M}+\mathrm{Na}]^{+}$found 244.0569.

Synthesis of methyl 2-(6-hydroxy-7-oxo-6,7-dihydro-5H-[1,3]dioxolo[4,5-f]isoindol-5-yl)acetate 4b<smiles>COC(=O)CC1c2cc3c(cc2C(=O)N1O)OCO3</smiles>

General procedure E was followed using methyl 3-(6-formylbenzo[d][1,3]dioxol-5-yl)propiolate $(0.339 \mathrm{~g}$, $1.46 \mathrm{mmol})$. Purified by column chromatography ( $5 \% \mathrm{MeOH}$ in $\mathrm{DCM})$ and concentrated in vacuo to give the title compound as a white solid $(0.241 \mathrm{~g}, 63 \%)$. mp $191{ }^{\circ} \mathrm{C} ;{ }^{1} \mathrm{H}$ NMR $\left(300 \mathrm{MHz}, \mathrm{CDCl}_{3}\right) \delta \mathrm{ppm} 10.28$ (br, 1H, N-OH), 7.14 (s, 1H, ArH), 6.90 (s, 1H, ArH), 6.04 (s, 2H, OCH $2 \mathrm{O}), 4.99$ (dd, J= 8.3, 4.4Hz, 1H, $\operatorname{ArCHN}), 3.76\left(\mathrm{~s}, 3 \mathrm{H}, \mathrm{OCH}_{3}\right), 3.26\left(\mathrm{dd}, J=16.4,4.4 \mathrm{~Hz}, 1 \mathrm{H}, \mathrm{CH}_{\mathrm{A}} \mathrm{CH}_{\mathrm{B}}\right), 2.59$ (dd, J=16.4, 8.5Hz, 1H, $\left.\mathrm{CH}_{\mathrm{A}} \mathrm{CH}_{\mathrm{B}}\right) ;{ }^{13} \mathrm{C} \mathrm{NMR}\left(75 \mathrm{MHz}, \mathrm{CDCl}_{3}\right) \delta \mathrm{ppm} 171.2,165.6,151.8,148.6,137.6,123.4,103.8,103.6,102.1$, 58.9, 52.3, 36.2; IR (thin film) $v_{\max }\left(\mathrm{cm}^{-1}\right)$ : broad peak at $2694.98(\mathrm{O}-\mathrm{H}), 1731.47(\mathrm{C}=\mathrm{O}), 1685.29(\mathrm{C}=\mathrm{O})$, 1293.29 and $1031.34\left(\mathrm{OCH}_{2}\right)$; HRMS (ESI): m/z calculated for $\mathrm{C}_{12} \mathrm{H}_{11} \mathrm{NO}_{6}: 266.0586$; for $[\mathrm{M}+\mathrm{H}]^{+}$found: 266.0656; for $[\mathrm{M}+\mathrm{Na}]^{+}$found: 288.0479

\section{Synthesis of methyl 2-(2-hydroxy-3-oxo-5-(trifluoromethyl)isoindolin-1-yl)acetate 4c}<smiles>COC(=O)CC1c2ccc(C(F)(F)F)cc2C(=O)N1O</smiles>

General procedure E was followed using methyl methyl 3-(2-formyl-4-(trifluoromethyl)phenyl)propiolate $(0.249 \mathrm{~g}, 9.7 \mathrm{mmol})$. Concentrated in vacuo to give the title compound $(0.256 \mathrm{~g}, 91 \%)$. mp $118-120{ }^{\circ} \mathrm{C} ;{ }^{1} \mathrm{H}$ NMR (500 MHz, $\left.\mathrm{CDCl}_{3}\right) \delta$ ppm 11.11 (s, br, 1H, OH), 7.99 (s, 1H, ArH), 7.79 (d, J = 8.0 Hz, 1H, ArH), $7.65(\mathrm{~d}, J=8.0 \mathrm{~Hz}, 1 \mathrm{H}, \mathrm{ArH}), 5.21(\mathrm{dd}, J=8.4,3.9 \mathrm{~Hz}, 1 \mathrm{H}, \mathrm{ArCHN}), 3.76\left(\mathrm{~s}, 3 \mathrm{H}, \mathrm{OCH}_{3}\right), 3.39$ (dd, $J=$ 16.8, 4.1 Hz, 1H, $\left.\mathrm{CH}_{\mathrm{A}} \mathrm{CH}_{\mathrm{B}}\right), 2.68\left(\mathrm{dd}, J=16.8,8.8 \mathrm{~Hz}, 1 \mathrm{H}, \mathrm{CH}_{\mathrm{A}} \mathrm{CH}_{\mathrm{B}}\right) ;{ }^{13} \mathrm{C} \mathrm{NMR}(126 \mathrm{MHz}, \mathrm{CDCl} 3) \delta$ ppm 170.6, 163.6, 145.1, $131.7\left(\mathrm{q},{ }^{2} J \mathrm{C}-\mathrm{F}=33.0 \mathrm{~Hz}\right), 130.8,129.1\left(\mathrm{q},{ }^{3} J \mathrm{C}-\mathrm{F}=3.4 \mathrm{~Hz}\right), 123.8,123.6(\mathrm{q}$, $\left.{ }^{1} J C-F=272.6 \mathrm{~Hz}\right), 120.8\left(\mathrm{q},{ }^{3} J \mathrm{C}-\mathrm{F}=3.8 \mathrm{~Hz}\right), 59.2,52.4,35.4$; IR (thin film) $v_{\max }\left(\mathrm{cm}^{-1}\right)$ : broad peak at 
$2717.93(\mathrm{O}-\mathrm{H}), 1727.89(\mathrm{C}=\mathrm{O}), 1697.12(\mathrm{C}=\mathrm{O}), 1326.84,1175.92$ and $1114.03\left(\mathrm{CF}_{3}\right) ; \mathrm{HRMS}(\mathrm{ESI}): \mathrm{m} / \mathrm{z}$ calculated for $\mathrm{C}_{12} \mathrm{H}_{10} \mathrm{~F}_{3} \mathrm{NO}_{4}$ : 290.0562; for $[\mathrm{M}+\mathrm{H}]^{+}$found: 290.0618 ; for $[\mathrm{M}+\mathrm{Na}]^{+}$found 312.0437 .

Synthesis of methyl 2-(2-hydroxy-3-oxo-5-fluoroisoindolin-1-yl)acetate 4d<smiles>COC(=O)CC1c2ccc(F)cc2C(=O)N1O</smiles>

General procedure E was followed using methyl 3-(2-formyl-4-fluorophenyl)propiolate (1.02 g, 4.95 mmol). Concentrated in vacuo obtaining the title compound as an orange solid $(1.122 \mathrm{~g}, 95 \%) . \mathrm{mp} 170{ }^{\circ} \mathrm{C}$; ${ }^{1} \mathrm{H}$ NMR (300 MHz, $\mathrm{CDCl}_{3}$ ) $\delta$ ppm 10.99 (s, br, 1H, OH), 7.47 (dd, $\left.J=8.4,4.5 \mathrm{~Hz}, 1 \mathrm{H}, \mathrm{ArH}\right), 7.42$ (dd, $J$ = 7.5, $2.4 \mathrm{~Hz}, 1 \mathrm{H}, \mathrm{ArH}), 7.27-7.18(\mathrm{~m}, 1 \mathrm{H}, \mathrm{ArH}), 5.11(\mathrm{dd}, J=8.6,4.3 \mathrm{~Hz}, 1 \mathrm{H}, \operatorname{ArCHN}), 3.77(\mathrm{~s}, 3 \mathrm{H}$, $\left.\mathrm{OCH}_{3}\right), 3.34\left(\mathrm{dd}, J=16.6,4.4 \mathrm{~Hz}, 1 \mathrm{H}, \mathrm{CH}_{\mathrm{A}} \mathrm{CH}_{\mathrm{B}}\right), 2.63\left(\mathrm{dd}, J=16.6,8.7 \mathrm{~Hz}, 1 \mathrm{H}, \mathrm{CH}_{\mathrm{A}} \mathrm{CH}_{\mathrm{B}}\right) ;{ }^{13} \mathrm{C} \mathrm{NMR}$ $\left(75 \mathrm{MHz}, \mathrm{CDCl}_{3}\right) \delta \mathrm{ppm}$ 170.9, 164.0, $163.1\left(\mathrm{~d},{ }^{1} J \mathrm{C}-\mathrm{F}=248.8 \mathrm{~Hz}\right), 137.3\left(\mathrm{~d},{ }^{4} J \mathrm{C}-\mathrm{F}=2.8 \mathrm{~Hz}\right), 131.8(\mathrm{~d}$, $\left.{ }^{3} J \mathrm{C}-\mathrm{F}=9.3 \mathrm{~Hz}\right), 124.8\left(\mathrm{~d},{ }^{3} \mathrm{JC}-\mathrm{F}=8.3 \mathrm{~Hz}\right), 119.6\left(\mathrm{~d},{ }^{2} J \mathrm{C}-\mathrm{F}=23.3 \mathrm{~Hz}\right), 110.6\left(\mathrm{~d},{ }^{2} J \mathrm{C}-\mathrm{F}=24.0 \mathrm{~Hz}\right), 58.9$, 52.4, 35.8; IR (thin film) $v_{\max }\left(\mathrm{cm}^{-1}\right)$ : br peak $2921.77(\mathrm{O}-\mathrm{H}), 1730.98(\mathrm{C}=\mathrm{O}), 1645.65(\mathrm{C}=\mathrm{O})$; HRMS (ESI): $\mathrm{m} / \mathrm{z}$ calculated for $\mathrm{C}_{11} \mathrm{H}_{10} \mathrm{FNO}_{4}: 240.0594$; for $[\mathrm{M}+\mathrm{H}]^{+}$found: 240.0662 ; for $[\mathrm{M}+\mathrm{Na}]^{+}$found 262.0491 .

Synthesis of methyl 2-(2-hydroxy-5,6-dimethoxy-3-oxo-2,3-dihydro-1H-isoindol-1-yl)acetate 4e<smiles>COC(=O)CC1c2cc(OC)c(OC)cc2C(=O)N1O</smiles>

Hydroxylamine solution $50 \%$ in $\mathrm{H}_{2} \mathrm{O}(0.104 \mathrm{~mL}, 1.1 \mathrm{eq})$ was added to a stirred mixture of methyl 3-(2formyl-4,5-dimethoxyphenyl)propiolate $37.6(0.354 \mathrm{~g}, 2.79 \mathrm{mmol}, 1 \mathrm{eq})$ and $\mathrm{CsCO}_{3}(2.32 \mathrm{~g}, 7.13 \mathrm{mmol}, 5$ eq) in $\operatorname{THF}(7.15 \mathrm{~mL})$ at $-20^{\circ} \mathrm{C}$ and then was allow to rise to room temperature. The reaction was allowed to stir for $3 \mathrm{hr}$. The mixture was filtered, neutralized with $\mathrm{HCl} 3 \mathrm{M}$ and extracted with EtOAc, dried over anhydrous $\mathrm{MgSO}_{4}$ and concentrated in vacuo to give the wanted compound as a red solid (0.36 g, 51\%); ${ }^{1} \mathrm{H}$ NMR $\left(300 \mathrm{MHz}, \mathrm{CDCl}_{3}\right.$ ) $\delta \mathrm{ppm} 7.19$ (s, 1H, ArH), 6.95 (s, 1H, ArH), 4.98 (dd, $J=8.6,4.5 \mathrm{~Hz}, 1 \mathrm{H}$, $\mathrm{ArCHN}), 3.90\left(\mathrm{~s}, 3 \mathrm{H}, \mathrm{OCH}_{3}\right), 3.88\left(\mathrm{~s}, 3 \mathrm{H}, \mathrm{OCH}_{3}\right), 3.74\left(\mathrm{~s}, 3 \mathrm{H}, \mathrm{OCH}_{3}\right), 3.27$ (dd, $J=16.5,4.6 \mathrm{~Hz}, 1 \mathrm{H}$, $\left.\mathrm{CH}_{\mathrm{A}} \mathrm{H}_{\mathrm{B}} \mathrm{CO}\right), 2.58\left(\mathrm{dd}, J=16.5,8.7 \mathrm{~Hz}, 1 \mathrm{H}, \mathrm{CH}_{\mathrm{A}} \mathrm{H}_{\mathrm{B}} \mathrm{CO}\right) ;{ }^{13} \mathrm{C} \mathrm{NMR}\left(75 \mathrm{MHz}, \mathrm{CDCl}_{3}\right) \delta \mathrm{ppm} 171.4,166.0$, 153.0, 150.1, 135.7, 121.6, 105.4, 105.3, 58.8, 56.4, 56.3, 52.2, 36.2; IR (thin film) $v_{\max }\left(\mathrm{cm}^{-1}\right)$ : br peak at $2952.84(\mathrm{O}-\mathrm{H}), 1729.22(\mathrm{C}=\mathrm{O}), 1673.16(\mathrm{C}=\mathrm{O}), 1290.93$ and $1112.89\left(\mathrm{OCH}_{3}\right) ; \mathrm{HRMS}(\mathrm{ESI}): \mathrm{m} / \mathrm{z}$ calculated for $\mathrm{C}_{13} \mathrm{H}_{15} \mathrm{NO}_{6}: 282.0899$; for $[\mathrm{M}+\mathrm{H}]^{+}$found: 282.0951 ; for $[\mathrm{M}+\mathrm{Na}]^{+}$found: 304.0743 . 


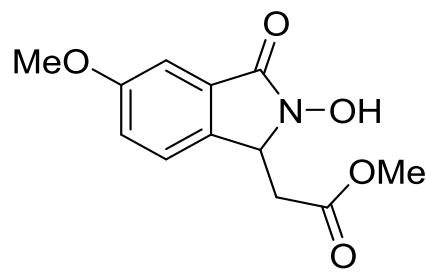

Hydroxylamine solution $50 \%$ in $\mathrm{H}_{2} \mathrm{O}(0.02 \mathrm{~mL}, 1.1 \mathrm{eq})$ was added to a stirred mixture of methyl 3-(2formyl-4-methoxyphenyl)propiolate $(0.609 \mathrm{~g}, 2.79 \mathrm{mmol}, 1 \mathrm{eq})$ and $\mathrm{CsCO} 3$ (4.55 g, $13.96 \mathrm{mmol}, 5 \mathrm{eq})$ in THF ( $14 \mathrm{~mL})$ at $-20^{\circ} \mathrm{C}$ and then was allow to rise to room temperature. The reaction was allowed to stir for 1hr. The mixture was filtered, neutralized with $\mathrm{HCl} 3 \mathrm{M}$ and extracted with EtOAc, dried with $\mathrm{MgSO}_{4}$ and concentrated in vacuo to give the wanted compound as a pale yellow solid (0.36 g, 51\%). mp 127-129 ${ }^{\circ} \mathrm{C}$; ${ }^{1} \mathrm{H}$ NMR $\left(300 \mathrm{MHz}, \mathrm{CDCl}_{3}\right) \delta \mathrm{ppm} 7.34$ (d, J=8.4 Hz, 1H, ArH), 7.23 (d, J=2.4 Hz, 1H, ArH), 7.06 (dd, $J=8.4,2.5 \mathrm{~Hz}, 1 \mathrm{H}, \mathrm{ArH}), 5.07(\mathrm{dd}, J=9.0,3.9 \mathrm{~Hz}, 1 \mathrm{H}, \mathrm{ArCHN}), 3.82\left(\mathrm{~s}, 3 \mathrm{H}, \mathrm{OCH}_{3}\right), 3.75\left(\mathrm{~s}, 3 \mathrm{H}, \mathrm{OCH}_{3}\right)$, $3.29\left(\mathrm{dd}, J=16.3,4.6 \mathrm{~Hz}, 1 \mathrm{H}, \mathrm{CH}_{\mathrm{A}} \mathrm{CH}_{\mathrm{B}}\right), 2.60\left(\mathrm{dd}, J=16.3,8.6 \mathrm{~Hz}, 1 \mathrm{H}, \mathrm{CH}_{\mathrm{A}} \mathrm{CH}_{\mathrm{B}}\right) ;{ }^{13} \mathrm{C} \mathrm{NMR}(75 \mathrm{MHz}$, $\left.\mathrm{CDCl}_{3}\right) \delta \mathrm{ppm} 171.2,165.0,160.4,133.9,131.0,123.9,120.0,106.8,58.8,55.8,52.3,36.2$; IR (thin film) $v_{\max }\left(\mathrm{cm}^{-1}\right)$ : br peak at $2950.85(\mathrm{OH}), 1732.87(\mathrm{C}=\mathrm{O}), 1677.16(\mathrm{C}=\mathrm{O}), 1021.81$ and $1282.07\left(\mathrm{OCH}_{3}\right)$; HRMS (ESI): $\mathrm{m} / \mathrm{z}$ calculated for $\mathrm{C}_{12} \mathrm{H}_{13} \mathrm{NO}_{5}$ : 252.0794; for $[\mathrm{M}+\mathrm{H}]^{+}$found: 252.0861 ; for $[\mathrm{M}+\mathrm{Na}]^{+}$found 274.0667.

Synthesis of methyl 2-(2-hydroxy-3-oxo-4-fluoroisoindolin-1-yl)acetate $4 \mathrm{~g}$<smiles>COC(=O)CC1c2cccc(F)c2C(=O)N1O</smiles>

General procedue E was followed using methyl 3-(2-formyl-3-fluorophenyl)propiolate (0.735 g, 3.57 mmol). Purified by column chromatography ( $2 \% \mathrm{MeOH}$ in DCM) and concentrated in vacuo to give the product as a white solid (0.56 g, 66\%). mp $168{ }^{\circ} \mathrm{C} ;{ }^{1} \mathrm{H}$ NMR $\left(500 \mathrm{MHz}, \mathrm{CD}_{3} \mathrm{OD}\right) \delta \mathrm{ppm} 7.63(\mathrm{td}, J=8.0$, $5.0 \mathrm{~Hz}, 1 \mathrm{H}, \mathrm{ArH}), 7.36$ (d, J=7.6 Hz, 1H, ArH), 7.20 (t, J=9.0 Hz, 1H, ArH), 5.05 (t, J=6.0 Hz, 1H, $\operatorname{ArCHN}), 3.69$ (s, 3H, $\left.\mathrm{OCH}_{3}\right), 3.04\left(\mathrm{dd}, J=16.4,5.5 \mathrm{~Hz}, 1 \mathrm{H}, \mathrm{CH}_{\mathrm{A}} \mathrm{H}_{\mathrm{B}} \mathrm{CO}\right), 2.89$ (dd, $J=16.4,6.5 \mathrm{~Hz}, 1 \mathrm{H}$, $\left.\mathrm{CH}_{\mathrm{A}} \mathrm{H}_{\mathrm{B}} \mathrm{CO}\right) ;{ }^{13} \mathrm{C} \mathrm{NMR}\left(75 \mathrm{MHz}, \mathrm{CD}_{3} \mathrm{OD}\right) \delta \mathrm{ppm} 172.1,163.5\left(\mathrm{~d},{ }^{3} J_{\mathrm{C}-\mathrm{F}}=2.2 \mathrm{~Hz}\right), 159.8\left(\mathrm{~d},{ }^{1} J_{\mathrm{C}-\mathrm{F}}=258.2\right.$ $\mathrm{Hz}), 145.8\left(\mathrm{~d},{ }^{3} J_{\mathrm{C}-\mathrm{F}}=2.9 \mathrm{~Hz}\right), 135.5\left(\mathrm{~d},{ }^{3} J_{\mathrm{C}-\mathrm{F}}=7.8 \mathrm{~Hz}\right), 120.1\left(\mathrm{~d},{ }^{4} J_{\mathrm{C}-\mathrm{F}}=4.2 \mathrm{~Hz}\right), 118.3\left(\mathrm{~d},{ }^{2} J_{\mathrm{C}-\mathrm{F}}=13.4 \mathrm{~Hz}\right)$, $117.0\left(\mathrm{~d},{ }^{2} J_{\mathrm{C}-\mathrm{F}}=19.4 \mathrm{~Hz}\right), 60.1,52.4,36.6$; IR (thin film) $v_{\max }\left(\mathrm{cm}^{-1}\right): 1711.64(\mathrm{C}=\mathrm{O}) ; \mathrm{HRMS}(\mathrm{ESI}): \mathrm{m} / \mathrm{z}$ calculated for $\mathrm{C}_{11} \mathrm{H}_{10} \mathrm{O}_{4} \mathrm{FN}$ : 240.0594; for $[\mathrm{M}+\mathrm{H}]^{+}$found: 240.0626 ; for $[\mathrm{M}+\mathrm{Na}]^{+}$found: 262.0469 . 


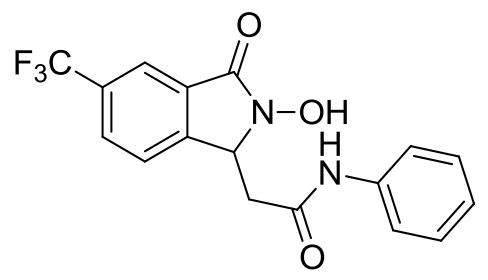

General procedue E was followed using 3-(2-formyl-4-(trifluoromethyl)phenyl)-N-phenylpropiolamide $(0.1 \mathrm{~g}, 0.4 \mathrm{mmol})$. Purified by column chromatography (2\% $\mathrm{MeOH}$ in DCM) and concentrated in vacuo to give the product as a white solid $(0.094 \mathrm{~g}, 85 \%)$. mp 240-242 ${ }^{\circ} \mathrm{C}$ (decomp); ${ }^{1} \mathrm{H}$ NMR (300MHz, DMSO) $\delta$ $=10.12(\mathrm{~s}, 1 \mathrm{H}, \mathrm{NH}), 8.01(\mathrm{~d}, J=7.2 \mathrm{~Hz}, 1 \mathrm{H}, \mathrm{ArH}), 7.96(\mathrm{~s}, 1 \mathrm{H}, \operatorname{ArH}), 7.79$ (d, J= $7.9 \mathrm{~Hz}, 1 \mathrm{H}, \operatorname{ArH})$, $7.59(\mathrm{dd}, J=1.1,8.7 \mathrm{~Hz}, 2 \mathrm{H}, \mathrm{ArH}), 7.31(\mathrm{t}, J=7.9 \mathrm{~Hz}, 2 \mathrm{H}, \mathrm{ArH}), 7.11-6.99(\mathrm{~m}, 1 \mathrm{H}, \operatorname{ArH}), 5.15$ (t, $J=$ $6.4 \mathrm{~Hz}, 1 \mathrm{H}, \mathrm{ArCHN}), 3.13\left(\mathrm{dd}, J=5.3,15.4 \mathrm{~Hz}, 1 \mathrm{H}, \mathrm{CH}_{\mathrm{A}} \mathrm{H}_{\mathrm{B}} \mathrm{CO}\right), 2.78(\mathrm{dd}, J=7.5,15.4 \mathrm{~Hz}, 1 \mathrm{H}$, $\left.\mathrm{CH}_{\mathrm{A}} \mathrm{H}_{\mathrm{B}} \mathrm{CO}\right) ;{ }^{13} \mathrm{C}$ NMR $(126 \mathrm{MHz}, \mathrm{DMSO}) \delta=167.6,161.3,146.3,138.8,131.4,129.4,129.1,128.7,128.4$ $\left(\mathrm{q},{ }^{3} J \mathrm{C}-\mathrm{F}=2.5 \mathrm{~Hz}\right), 123.9\left(\mathrm{q},{ }^{2} J \mathrm{C}-\mathrm{F}=121.0 \mathrm{~Hz}\right), 119.2,119.2\left(\mathrm{q},{ }^{3} J \mathrm{C}-\mathrm{F}=3.8 \mathrm{~Hz}\right), 58.7,37.7$; IR (thin film) $v_{\max }\left(\mathrm{cm}^{-1}\right): 3319.57(\mathrm{NH}), 1704.51$ and $1684.07(\mathrm{C}=\mathrm{O})$; HRMS (ESI): $\mathrm{m} / \mathrm{z}$ calculated for $\mathrm{C}_{17} \mathrm{H}_{13} \mathrm{~F}_{3} \mathrm{~N}_{2} \mathrm{O}_{3}$ : 351.0956 ; for $[\mathrm{M}+\mathrm{H}]^{+}$found: 351.0938; for $[\mathrm{M}+\mathrm{Na}]^{+}$found: 373.0761 .

\section{Synthesis of ethyl 2-(3-oxoisoindolin-1-yl)acetate 15a}

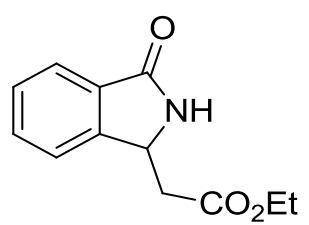

$\mathrm{RuCl}_{3} \cdot 3 \mathrm{H}_{2} \mathrm{O}(0.009 \mathrm{~g}, 0.034 \mathrm{mmol})$ and $\mathrm{Zn}-\mathrm{Cu}$ couple $(0.026 \mathrm{~g}, 0.2 \mathrm{mmol})$ were added to methyl 2-(2hydroxy-3-oxoisoindolin-1-yl)acetate $(0.15 \mathrm{~g}, 0.67 \mathrm{mmol})$ in ethanol $(5 \mathrm{~mL})$ under a $\mathrm{N}_{2}$ atmosphere and heated at $90{ }^{\circ} \mathrm{C}$ for $24 \mathrm{~h}$. The reaction was cooled and filtered through celite, the solvent was removed in vacuo and the residue was extracted with EtOAc and filtered again through celite. The reaction was concentrated in vacuo to give the title compound as a dark red oil $(0.1 \mathrm{~g}, 66 \%) ;{ }^{1} \mathrm{H} \mathrm{NMR}(300 \mathrm{MHz}$, METHANOL- $\left.d_{4}\right) \delta$ ppm $7.78(\mathrm{~d}, J=7.54 \mathrm{~Hz}, 1 \mathrm{H}, \mathrm{ArH}) 7.45-7.72(\mathrm{~m}, 3 \mathrm{H}, \mathrm{ArH}) 5.02$ (dd, $J=8.10,4.90$ $\mathrm{Hz}, 1 \mathrm{H}, \mathrm{CH}) 4.15$ (q, J=6.00 Hz, $2 \mathrm{H}, \mathrm{CH}) 3.00$ (dd, J=16.39, $4.90 \mathrm{~Hz}, 1 \mathrm{H}, \mathrm{CH}) 2.63$ (dd, J=16.39, 8.10 $\mathrm{Hz}, 1 \mathrm{H}, \mathrm{CH}) 1.26(\mathrm{t}, J=9.00 \mathrm{~Hz}, 3 \mathrm{H}, \mathrm{CH}) ;{ }^{13} \mathrm{C} \mathrm{NMR}\left(75 \mathrm{MHz}, \mathrm{MeOD}-\mathrm{d}_{4}\right) \delta \mathrm{ppm} 172.9,172.3,148.3$, 133.6, 133.0, 129.8, 124.5, 124.2, 62.1, 55.0, 40.1, 14.6; I.R (thinfilm) vmax (cm-1): 3591 (N-H), 1704 (O$\mathrm{C}=\mathrm{O}), 1654(\mathrm{C}=\mathrm{O})$; HRMS (ESI): m/z calculated for $\mathrm{C}_{12} \mathrm{H}_{13} \mathrm{NO}_{3}$ : requires: 242.0793 for $[\mathrm{M}+\mathrm{Na}]^{+}$; found: 242.0782 . 
<smiles>CCOC(=O)CC1NC(=O)c2cc3c(cc21)OCO3</smiles>

$\mathrm{RuCl}_{3} \cdot 3 \mathrm{H}_{2} \mathrm{O}(0.030 \mathrm{~g}, 0.15 \mathrm{mmol}, 0.05 \mathrm{eq})$ and $\mathrm{Zn}$-Cu couple $(0.057 \mathrm{~g}, 0.88 \mathrm{mmol}, 0.3 \mathrm{eq})$ were added to methyl 2-(6-hydroxy-7-oxo-6,7-dihydro-5H-[1,3]dioxolo[4,5-f]isoindol-5-yl)acetate (0.777 g, $2.93 \mathrm{mmol}$, 1 eq) in ethanol $(90 \mathrm{~mL})$ under a $\mathrm{N}_{2}$ atmosphere and heated at $90{ }^{\circ} \mathrm{C}$ for $24 \mathrm{~h}$. The reaction was cooled and filtered through celite, the solvent removed in vacuo and the residue was disolved in EtOAc and filtered again through celite. The residue was purified by column chromatography (5\% $\mathrm{MeOH}$ in $\mathrm{DCM})$ and concentrated in vacuo to give the product as a white solid $(0.391 \mathrm{~g}, 51 \%)$. mp 201-203 ${ }^{\circ} \mathrm{C} ;{ }^{1} \mathrm{H}$ NMR $(300$ $\left.\mathrm{MHz}, \mathrm{CDCl}_{3}\right) \delta \mathrm{ppm} 7.21$ (s, 1H, ArH), $6.80(\mathrm{~s}, 1 \mathrm{H}, \mathrm{ArH}), 6.76(\mathrm{~s}, 1 \mathrm{H}, \mathrm{NH}), 6.06\left(\mathrm{~s}, 2 \mathrm{H}, \mathrm{OCH}_{2} \mathrm{O}\right), 4.80$ (dd, $J=10.3,3.6 \mathrm{~Hz}, 1 \mathrm{H}, \mathrm{ArCHN}), 4.22\left(\mathrm{q}, J=7.1 \mathrm{~Hz}, 2 \mathrm{H}, \mathrm{OCH}_{2} \mathrm{CH}_{3}\right), 2.91$ (dd, $J=16.9,3.7 \mathrm{~Hz}, 1 \mathrm{H}$, $\left.\mathrm{CH}_{\mathrm{A}} \mathrm{CH}_{\mathrm{B}}\right), 2.43\left(\mathrm{dd}, J=16.9,10.3 \mathrm{~Hz}, 1 \mathrm{H}, \mathrm{CH}_{\mathrm{A}} \mathrm{CH}_{\mathrm{B}}\right), 1.29\left(\mathrm{t}, J=7.1 \mathrm{~Hz}, 3 \mathrm{H}, \mathrm{OCH}_{2} \mathrm{CH}_{3}\right) ;{ }^{13} \mathrm{C} \mathrm{NMR}(75$ $\left.\mathrm{MHz}, \mathrm{CDCl}_{3}\right) \delta \mathrm{ppm} 171.23,169.8,151.9,148.9,141.7,126.0,103.8,102.7,102.3,61.4,52.6,39.8,14.3$; IR (thin film) $v_{\max }\left(\mathrm{cm}^{-1}\right): 1723.49(\mathrm{C}=\mathrm{O}), 1700.91(\mathrm{C}=\mathrm{O}), 1240.89$ and $1038.96\left(\mathrm{OCH}_{2}\right)$; HRMS (ESI): $\mathrm{m} / \mathrm{z}$ calculated for $\mathrm{C}_{13} \mathrm{H}_{13} \mathrm{NO}_{5}$ : 264.0794; for $[\mathrm{M}+\mathrm{H}]^{+}$found: 264.0876 ; for $[\mathrm{M}+\mathrm{Na}]^{+}$found: 286.0668

Synthesis of methyl 2-(3-oxo-5-(trifluoromethyl)isoindolin-1-yl)acetate 15c<smiles>COC(=O)CC1NC(=O)c2cc(C(F)(F)F)ccc21</smiles>

$\mathrm{Et}_{3} \mathrm{~N}(0.426 \mathrm{~mL}, 3.06 \mathrm{mmol}, 2 \mathrm{eq})$ and diethyl phosphorocyanidate $(0.348 \mathrm{~mL}, 2.30 \mathrm{mmol}, 1.5 \mathrm{eq})$ were added to a solution of methyl 2-(2-hydroxy-3-oxo-5-(trifluoromethyl)isoindolin-1-yl)acetate (0.442 g, 1.53 mmol) in THF ( $15 \mathrm{~mL}$ ) under an $\mathrm{N}_{2}$ atmosphere and the mixture stirred at $\mathrm{rt}$ for 30 minutes. The reaction was diluted with water $(10 \mathrm{~mL})$ and the mixture extracted with $\mathrm{Et}_{2} \mathrm{O}(3 \times 10 \mathrm{~mL})$. The organic layers were combined, dried with $\mathrm{MgSO}_{4}$ and concentrated in vacuo. The residue was disolved in anhydrous THF (18.4 $\mathrm{mL}$ ) under an $\mathrm{N}_{2}$ atmosphere, and $\mathrm{SmI}_{2}(0.1 \mathrm{M}$ in THF) $(61.2 \mathrm{~mL}, 6.12 \mathrm{mmol}, 4 \mathrm{eq})$ added to the solution, which was then stirred for $1 \mathrm{hr}$ at rt. The reaction was quenched with saturated $\mathrm{NaHCO}_{3}$ and extracted with EtOAc and washed with brine, the organic layers were combined, dried with $\mathrm{MgSO}_{4}$ and concentrated in vacuo. Purification by column chromatography ( $5 \% \mathrm{MeOH}$ in $\mathrm{DCM}$ ) afforded the title compound as a pale yellow solid (0.295 g, 71\%). mp 173-175 ${ }^{\circ} \mathrm{C} ;{ }^{1} \mathrm{H}$ NMR $\left(300 \mathrm{MHz}, \mathrm{CDCl}_{3}\right) \delta \mathrm{ppm} 8.13$ (s, $\left.1 \mathrm{H}, \mathrm{ArH}\right), 7.84$ $(\mathrm{dd}, J=8.0,1.0 \mathrm{~Hz}, 1 \mathrm{H}, \mathrm{ArH}), 7.57(\mathrm{dd}, J=8.0,0.5 \mathrm{~Hz}, 1 \mathrm{H}), 7.43(\mathrm{~s}, 1 \mathrm{H}, \mathrm{NH}), 5.02(\mathrm{dd}, J=10.0,3.8 \mathrm{~Hz}$, $1 \mathrm{H}, \mathrm{ArCH}), 3.79\left(\mathrm{~s}, 3 \mathrm{H}, \mathrm{OCH}_{3}\right), 3.03\left(\mathrm{dd}, J=17.0,4.0 \mathrm{~Hz}, 1 \mathrm{H}, \mathrm{CH}_{\mathrm{A}} \mathrm{CH}_{\mathrm{B}}\right), 2.54(\mathrm{dd}, J=17.0,10.1 \mathrm{~Hz}, 1 \mathrm{H}$, $\mathrm{CH}_{\mathrm{A}} \mathrm{CH}_{\mathrm{B}}$ ); ${ }^{13} \mathrm{C}$ NMR $\left(75 \mathrm{MHz}, \mathrm{CDCl}_{3}\right) \delta \mathrm{ppm} 171.3,168.8,149.2,132.9,131.7$ (q, $\left.{ }^{2} J \mathrm{C}-\mathrm{F}=33.0 \mathrm{~Hz}\right), 129.1$ $\left(\mathrm{q},{ }^{3} J \mathrm{C}-\mathrm{F}=3.5 \mathrm{~Hz}\right), 123.8\left(\mathrm{q},{ }^{1} J \mathrm{C}-\mathrm{F}=272.6 \mathrm{~Hz}\right), 123.3,121.6\left(\mathrm{q},{ }^{3} J \mathrm{C}-\mathrm{F}=3.9 \mathrm{~Hz}\right), 53.1,52.5,39.1$; IR 
(thin film) $v_{\max }\left(\mathrm{cm}^{-1}\right): 1728.17(\mathrm{C}=\mathrm{O}), 1702.13(\mathrm{C}=\mathrm{O}), 1321.88$ and $1115.27\left(\mathrm{CF}_{3}\right) ; \mathrm{HRMS}(\mathrm{ESI}): \mathrm{m} / \mathrm{z}$ calculated for $\mathrm{C}_{12} \mathrm{H}_{10} \mathrm{~F}_{3} \mathrm{NO}_{3}$ : requires: 274.0612 for $[\mathrm{M}+\mathrm{H}]^{+}$; found: 274.0365 .

Synthesis of methyl 2-(5-fluoro-3-oxoisoindolin-1-yl)acetate 15d<smiles>COC(=O)CC1NC(=O)c2cc(F)ccc21</smiles>

$\mathrm{Et}_{3} \mathrm{~N}(0.450 \mathrm{~mL}, 3.23 \mathrm{mmol}, 2 \mathrm{eq})$ and diethyl phosphorocyanidate $(0.366 \mathrm{~mL}, 2.42 \mathrm{mmol}, 1.5 \mathrm{eq})$ were added to a solution of methyl 2-(2-hydroxy-3-oxo-5-fluoroisoindolin-1-yl)acetate $(0.386 \mathrm{~g}, 1.61 \mathrm{mmol})$ in THF $(16.0 \mathrm{~mL})$ under an $\mathrm{N}_{2}$ atmosphere and the mixture stirred at $\mathrm{rt}$ for 45 minutes. The reaction was diluted with water $(10 \mathrm{~mL})$ and extracted with $\mathrm{Et}_{2} \mathrm{O}(3 \times 10 \mathrm{~mL})$. The organic layers were combined, dried with $\mathrm{MgSO}_{4}$ and concentrated in vacuo. The residue was disolved in anhydrous THF (19.3 mL) under a $\mathrm{N}_{2}$ atmosphere followed by addition of $\mathrm{SmI}_{2}(0.1 \mathrm{M}$ in THF) $(64.4 \mathrm{~mL}, 6.44 \mathrm{mmol}, 4 \mathrm{eq})$. The solution was stirred at $\mathrm{rt}$ for $1 \mathrm{~h}$ the reaction was then quenched with saturated $\mathrm{NaHCO}_{3}$ and extracted with EtOAc. The organic layers were then washed with brine, dried with $\mathrm{MgSO}_{4}$ and concentrated in vacuo to give the product as a white solid $(0.182 \mathrm{~g}, 51 \%)$. $\mathrm{mp} 164-165{ }^{\circ} \mathrm{C} ;{ }^{1} \mathrm{H}$ NMR $\left(300 \mathrm{MHz}, \mathrm{CDCl}_{3}\right) \delta \mathrm{ppm} 7.51(\mathrm{dd}, J=$ 7.5, 2.4 Hz, 1H, ArH), 7.39 (dd, $J=8.3,4.4 \mathrm{~Hz}, 1 \mathrm{H}, \mathrm{ArH}), 7.31$ (s, br, $1 \mathrm{H}, \mathrm{NH}), 7.27$ (td, $J=8.4,2.4 \mathrm{~Hz}$, $1 \mathrm{H}, \mathrm{ArH}), 4.92(\mathrm{dd}, J=9.5,3.9 \mathrm{~Hz}, 1 \mathrm{H}, \mathrm{ArCHN}), 3.78\left(\mathrm{~s}, 3 \mathrm{H}, \mathrm{OCH}_{3}\right), 2.98(\mathrm{dd}, J=17.0,3.9 \mathrm{~Hz}, 1 \mathrm{H}$, $\left.\mathrm{CH}_{\mathrm{A}} \mathrm{CH}_{\mathrm{B}}\right), 2.49\left(\mathrm{dd}, J=17.0,10.1 \mathrm{~Hz}, 1 \mathrm{H}, \mathrm{CH}_{\mathrm{A}} \mathrm{CH}_{\mathrm{B}}\right) ;{ }^{13} \mathrm{C} \mathrm{NMR}\left(75 \mathrm{MHz}, \mathrm{CDCl}_{3}\right) \delta \mathrm{ppm} 171.5,169.2(\mathrm{~d}$, $\left.{ }^{4} J \mathrm{C}-\mathrm{F}=3.4 \mathrm{~Hz}\right), 163.3\left(\mathrm{~d},{ }^{1} J \mathrm{C}-\mathrm{F}=248.3 \mathrm{~Hz}\right), 141.4\left(\mathrm{~d},{ }^{4} J \mathrm{C}-\mathrm{F}=2.5 \mathrm{~Hz}\right), 134.2\left(\mathrm{~d},{ }^{3} J \mathrm{C}-\mathrm{F}=8.6 \mathrm{~Hz}\right), 124.1$ $\left(\mathrm{d},{ }^{3} J \mathrm{C}-\mathrm{F}=8.5 \mathrm{~Hz}\right), 119.7\left(\mathrm{~d},{ }^{2} J \mathrm{C}-\mathrm{F}=23.8 \mathrm{~Hz}\right), 111.0\left(\mathrm{~d},{ }^{2} J \mathrm{C}-\mathrm{F}=23.5 \mathrm{~Hz}\right), 52.7,52.4,39.4$; IR (thin film) $v_{\max }\left(\mathrm{cm}^{-1}\right): 1711.85(\mathrm{C}=\mathrm{O})$; HRMS (ESI): $\mathrm{m} / \mathrm{z}$ calculated for $\mathrm{C}_{11} \mathrm{H}_{10} \mathrm{FNO}_{3}: 224.0645$; for $[\mathrm{M}+\mathrm{H}]^{+}$found: 224.0720 .

\section{Synthesis of methyl 2-(7-fluoro-1-oxoisoindolin-3-yl)acetate 15e}<smiles>COC(=O)CC1NC(=O)c2c(F)cccc21</smiles>

$\mathrm{Et}_{3} \mathrm{~N}(0.307 \mathrm{~mL}, 2.20 \mathrm{mmol}, 2 \mathrm{eq})$ and diethyl phosphorocyanidate $(0.250 \mathrm{~mL}, 1.65 \mathrm{mmol}, 1.5 \mathrm{eq})$ were added to a solution of methyl 2-(2-hydroxy-3-oxo-4-fluoroisoindolin-1-yl)acetate $(0.263 \mathrm{~g}, 1.10 \mathrm{mmol})$ in a mixture of THF $(11 \mathrm{~mL})$ and DMF $(1 \mathrm{~mL})$ under an $\mathrm{N}_{2}$ atmosphere and the reaction was stirred at $\mathrm{rt}$ for $30 \mathrm{~min}$. The reaction was then diluted with water $\left(10 \mathrm{mLand}\right.$ extracted with $\mathrm{Et}_{2} \mathrm{O}(3 \times 10 \mathrm{~mL})$. The organic layers were combined, dried with $\mathrm{MgSO}_{4}$ and concentrated in vacuo. The residue was disolved in anhydrous THF (13.2 mL) under a $\mathrm{N}_{2}$ atmosphere followed by addition of $\mathrm{SmI}_{2}$ (0.1 M in THF) (44.0 mL, $4.40 \mathrm{mmol}$, $4 \mathrm{eq}$ ). The reaction was then stirred at room temperature for $1 \mathrm{hr}$. The reaction was quenched with saturated 
$\mathrm{NaHCO}_{3}$ and extracted with EtOAc. The organic layers were then washed with brine, dried with $\mathrm{MgSO}_{4}$ and concentrated in vacuo to give the product as a white solid $(0.177 \mathrm{~g}, 72 \%)$. mp $125{ }^{\circ} \mathrm{C} ;{ }^{1} \mathrm{H}$ NMR $(500$ $\left.\mathrm{MHz}, \mathrm{CDCl}_{3}\right) \delta 7.57-7.50(\mathrm{~m}, 1 \mathrm{H}, \mathrm{ArH}), 7.47(\mathrm{~s}, 1 \mathrm{H}, \mathrm{NH}), 7.20(\mathrm{~d}, J=7.5 \mathrm{~Hz}, 1 \mathrm{H}, \mathrm{ArH}), 7.09$ (t, $J=8.7$ $\mathrm{Hz}, 1 \mathrm{H}, \mathrm{ArH}), 4.94(\mathrm{dd}, J=9.7,3.9 \mathrm{~Hz}, 1 \mathrm{H}, \mathrm{ArCHN}), 3.77$ (s, 3H, $\left.\mathrm{OCH}_{3}\right), 2.96(\mathrm{dd}, J=16.9,4.0 \mathrm{~Hz}, 1 \mathrm{H}$, $\left.\mathrm{CH}_{\mathrm{A}} \mathrm{H}_{\mathrm{B}} \mathrm{CO}\right), 2.54\left(\mathrm{dd}, J=16.9,9.8 \mathrm{~Hz}, 1 \mathrm{H}, \mathrm{CH}_{\mathrm{A}} \mathrm{H}_{\mathrm{B}} \mathrm{CO}\right) ;{ }^{13} \mathrm{C}$ NMR $\left(126 \mathrm{MHz}, \mathrm{CDCl}_{3}\right) \delta 171.3,167.5$, $159.3\left(\mathrm{~d},{ }^{1} J_{\mathrm{C}-\mathrm{F}}=260.9 \mathrm{~Hz}\right), 148.9\left(\mathrm{~d},{ }^{3} J_{\mathrm{C}-\mathrm{F}}=2.8 \mathrm{~Hz}\right), 134.3\left(\mathrm{~d},{ }^{3} J_{\mathrm{C}-\mathrm{F}}=7.7 \mathrm{~Hz}\right), 119.3\left(\mathrm{~d},{ }^{2} J_{\mathrm{C}-\mathrm{F}}=13.4 \mathrm{~Hz}\right)$, $118.5\left(\mathrm{~d},{ }^{4} J_{\mathrm{C}-\mathrm{F}}=4.2 \mathrm{~Hz}\right), 116.1\left(\mathrm{~d},{ }^{2} J_{\mathrm{C}-\mathrm{F}}=19.4 \mathrm{~Hz}\right), 52.9,52.4,39.5$; IR (thin film) $v_{\max }\left(\mathrm{cm}^{-1}\right): 1734.79$, $1684.76\left(\mathrm{C}=\mathrm{O}\right.$ ); HRMS (ESI): $\mathrm{m} / \mathrm{z}$ calculated for $\mathrm{C}_{11} \mathrm{H}_{10} \mathrm{O}_{3} \mathrm{FN}$ : 224.0645; for $[\mathrm{M}+\mathrm{H}]^{+}$found: 224.0708 ; for $[\mathrm{M}+\mathrm{Na}]^{+}$found: 246.0534

\section{Synthesis of tert-butyl 5-fluoro-1-(2-methoxy-2-oxoethyl)-3-oxoisoindoline-2-carboxylate 16}<smiles>COC(=O)CC1c2ccc(F)cc2C(=O)N1C(=O)OC(C)(C)C</smiles>

A mixture of methyl 2-(5-fluoro-3-oxoisoindolin-1-yl)acetate $(0.054 \mathrm{~g}, \quad 0.24 \mathrm{mmol}, \quad 1 \mathrm{eq}), \quad 4$ (dimethylamino)pyridine $(0.003 \mathrm{~g}, 0.02 .4 \mathrm{mmol}, 0.1 \mathrm{eq})$ and di-tert-butyl dicarbonate $(0.132 \mathrm{~g}, 0.61 \mathrm{mmol}$, $2.5 \mathrm{eq})$ in THF ( $2 \mathrm{~mL})$ was stirred at room temperature for $16 \mathrm{~h}$. Evaporation of the solvent in vacuo followed by column chromatography (2\% EtOH in DCM) afforded the title compound as a yellow oil (0.076 g, $97 \%$ ); ${ }^{1} \mathrm{H}$ NMR (300 MHz, $\left.\mathrm{CDCl}_{3}\right) \delta \mathrm{ppm} 7.54-7.45$ (m, 2H, ArH), $7.30(\mathrm{td}, J=8.6,2.5 \mathrm{~Hz}$, $1 \mathrm{H}, \mathrm{ArH}), 5.37$ (dd, $J=8.5,3.2 \mathrm{~Hz}, 1 \mathrm{H}, \mathrm{ArCHN}), 3.68\left(\mathrm{~s}, 3 \mathrm{H}, \mathrm{OCH}_{3}\right), 3.30(\mathrm{dd}, J=16.1,3.3 \mathrm{~Hz}, 1 \mathrm{H}$, $\mathrm{CH}_{\mathrm{A}} \mathrm{CH}_{\mathrm{B}}$ ), $2.68\left(\mathrm{dt}, J=19.7,9.8 \mathrm{~Hz}, 1 \mathrm{H}, \mathrm{CH}_{\mathrm{A}} \mathrm{CH}_{\mathrm{B}}\right), 1.58$ (s, 9H, BOC); ${ }^{13} \mathrm{C} \mathrm{NMR}\left(75 \mathrm{MHz}, \mathrm{CDCl}_{3}\right) \delta \mathrm{ppm}$ 170.6, $165.1\left(\mathrm{~d},{ }^{4} \mathrm{JC}-\mathrm{F}=3.8 \mathrm{~Hz}\right), 163.3\left(\mathrm{~d},{ }^{1} \mathrm{JC}-\mathrm{F}=249.3 \mathrm{~Hz}\right), 150.0,140.3\left(\mathrm{~d},{ }^{4} \mathrm{JC}-\mathrm{F}=2.5 \mathrm{~Hz}\right), 132.7$ (d, $\left.{ }^{3} J \mathrm{C}-\mathrm{F}=8.7 \mathrm{~Hz}\right), 125.0\left(\mathrm{~d},{ }^{3} \mathrm{JC}-\mathrm{F}=8.3 \mathrm{~Hz}\right), 121.7\left(\mathrm{~d},{ }^{2} \mathrm{JC}-\mathrm{F}=23.6 \mathrm{~Hz}\right), 111.5\left(\mathrm{~d},{ }^{2} J \mathrm{C}-\mathrm{F}=23.5 \mathrm{~Hz}\right), 84.0$, 56.4, 52.1, 38.5, 28.2; IR (thin film) $v_{\max }\left(\mathrm{cm}^{-1}\right): 1722.83(\mathrm{C}=\mathrm{O})$; HRMS (ESI): $\mathrm{m} / \mathrm{z}$ calculated for $\mathrm{C}_{16} \mathrm{H}_{18} \mathrm{FNO}_{5}: 324.1169$; for $[\mathrm{M}+\mathrm{H}]^{+}$found: 324.1225 ; for $[\mathrm{M}+\mathrm{Na}]^{+}$found 346.1046

\section{Synthesis of tert-butyl 5-fluoro-1-(2-methoxy-2-oxoethyl)isoindoline-2-carboxylate 18}<smiles>COC(=O)CC1c2ccc(F)cc2CN1C(=O)OC(C)(C)C</smiles>

$\mathrm{LiEt}_{3} \mathrm{BH}$ (1 $\mathrm{M}$ in THF) $(0.588 \mathrm{~mL}$ ) was added to a solution of tert-butyl 5-fluoro-1-(2-methoxy-2oxoethyl)-3-oxoisoindoline-2-carboxylate $(0.076 \mathrm{~g}, 0.235 \mathrm{mmol})$ in $\mathrm{THF}(2.4 \mathrm{~mL})$ at $-78^{\circ} \mathrm{C}$ under nitrogen 
atmosphere. After $30 \mathrm{~min}$, the reaction mixture was quenched with saturated $\mathrm{NaHCO}_{3}(1 \mathrm{~mL})$ and warmed to $0{ }^{\circ} \mathrm{C} .30 \% \mathrm{H}_{2} \mathrm{O}_{2}$ (3 drops) was added and the mixture was stirred at $0{ }^{\circ} \mathrm{C}$. After $20 \mathrm{~min}$, the organic solvent was removed in vacuo and the aqueous layer was extracted with $\mathrm{CH}_{2} \mathrm{Cl}_{2}(3 \times 10 \mathrm{~mL})$. The combined organic layers were dried over $\mathrm{MgSO}_{4}$, filtered and concentrated. The $\mathrm{N}$-Boc-carbinolamine product was obtained as a mixture of diastereomers and was used without purification. $\mathrm{BF}_{3} \cdot \mathrm{OEt}_{2}(0.032 \mathrm{~mL}, 0.25 \mathrm{mmol}$, $1.06 \mathrm{eq})$ was then added dropwise to a solution of crude $\mathrm{N}$-Boc-carbinolamine $(0.235 \mathrm{mmol})$ and $\mathrm{Et}_{3} \mathrm{SiH}$ ( $0.038 \mathrm{~mL}, 0.235 \mathrm{mmol})$ in $\mathrm{CH}_{2} \mathrm{Cl}_{2}(2.35 \mathrm{~mL})$ cooled to $-78^{\circ} \mathrm{C}$ under a $\mathrm{N}_{2}$ atmosphere for 30 minutes. A further portion of $\mathrm{Et}_{3} \mathrm{SiH}(0.038 \mathrm{~mL}, 0.235 \mathrm{mmol})$ and $\mathrm{BF}_{3} \cdot \mathrm{OEt}_{2}(0.032 \mathrm{~mL}, 0.25 \mathrm{mmol})$ was added and the resulting mixture was stirred for $2.5 \mathrm{hr}$ at $-78^{\circ} \mathrm{C}$. The reaction mixture was quenched with saturated aqueus $\mathrm{NaHCO}_{3}$, extracted with $\mathrm{CH}_{2} \mathrm{Cl}_{2}$ and dried over $\mathrm{MgSO}_{4}$. Evaporation of the solvent in vacuo and purification by column chromatography (2\% EtOH in DCM) afforded the title compound as a colorless oil as a mixture of rotamers $(0.042 \mathrm{~g}, 58 \%) ;{ }^{1} \mathrm{H}$ NMR $\left(300 \mathrm{MHz}, \mathrm{CDCl}_{3}\right) \delta \mathrm{ppm} 7.25-7.12(\mathrm{~m}, 1 \mathrm{H}, \mathrm{ArH})$, $7.01-6.84(\mathrm{~m}, 2 \mathrm{H}, \mathrm{ArH}), 5.30(\mathrm{dd}, J=12.0,10.1 \mathrm{~Hz}, 1 \mathrm{H}, \operatorname{ArCHN}), 4.65(\mathrm{dt}, J=28.7,15.7 \mathrm{~Hz}, 2 \mathrm{H}$, $\left.\mathrm{ArCH}_{2} \mathrm{~N}\right), 3.64+3.62\left(2 \mathrm{~s}, 3 \mathrm{H}, \mathrm{OCH}_{3}\right), 3.16-2.94\left(\mathrm{~m}, 1 \mathrm{H}, \mathrm{CH}_{\mathrm{A}} \mathrm{CH}_{\mathrm{B}}\right), 2.77$ (ddd, J = 26.3, 15.6, 7.6 Hz, $\left.1 \mathrm{H}, \mathrm{CH}_{\mathrm{A}} \mathrm{CH}_{\mathrm{B}}\right), 1.51+1.50(2 \mathrm{~s}, 9 \mathrm{H}, \mathrm{BOC}) ;{ }^{13} \mathrm{C} \mathrm{NMR}\left(75 \mathrm{MHz}, \mathrm{CDCl}_{3}\right) \delta \mathrm{ppm} 171.4,171.3,162.9\left(\mathrm{~d},{ }^{1} J \mathrm{C}-\right.$ $\mathrm{F}=245.3 \mathrm{~Hz}), 154.1,154.0,139.2\left(\mathrm{~d},{ }^{3} J \mathrm{C}-\mathrm{F}=8.6 \mathrm{~Hz}\right), 138.8\left(\mathrm{~d},{ }^{3} \mathrm{JC}-\mathrm{F}=8.8 \mathrm{~Hz}\right), 136.0\left(\mathrm{~d},{ }^{4} J \mathrm{C}-\mathrm{F}=2.3\right.$ $\mathrm{Hz}), 136.0\left(\mathrm{~d},{ }^{4} J \mathrm{C}-\mathrm{F}=1.9 \mathrm{~Hz}\right), 124.3\left(\mathrm{~d},{ }^{3} J \mathrm{C}-\mathrm{F}=9.0 \mathrm{~Hz}\right), 124.1\left(\mathrm{~d},{ }^{3} J \mathrm{C}-\mathrm{F}=9.2 \mathrm{~Hz}\right), 114.9\left(\mathrm{~d},{ }^{2} J \mathrm{C}-\mathrm{F}=\right.$ $22.8 \mathrm{~Hz}), 110.0\left(\mathrm{~d},{ }^{2} J \mathrm{C}-\mathrm{F}=17.2 \mathrm{~Hz}\right), 109.7\left(\mathrm{~d},{ }^{2} J \mathrm{C}-\mathrm{F}=17.3 \mathrm{~Hz}\right), 80.6,80.2,59.3,59.2,52.1\left(\mathrm{~d},{ }^{4} J \mathrm{C}-\mathrm{F}=\right.$ $2.4 \mathrm{~Hz}), 51.8\left(\mathrm{~d},{ }^{4} \mathrm{JC}-\mathrm{F}=4.8 \mathrm{~Hz}\right), 40.5,39.5$, 28.6; IR (thin film) $v_{\max }\left(\mathrm{cm}^{-1}\right): 1737.12(\mathrm{C}=\mathrm{O})$ and 1694.96 $(\mathrm{C}=\mathrm{O})$; HRMS (ESI): $\mathrm{m} / \mathrm{z}$ calculated for $\mathrm{C}_{16} \mathrm{H}_{20} \mathrm{FNO}_{4}: 310.1376$; for $[\mathrm{M}+\mathrm{H}]^{+}$found: 310.1431 ; for $[\mathrm{M}+\mathrm{Na}]^{+}$ found 332.1258

Synthesis of 7-fluoro-3-(2-hydroxyethyl)isoindolin-1-one 19

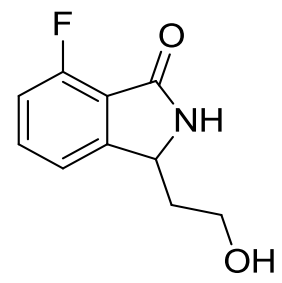

$\mathrm{LiBH}_{4}(0.033 \mathrm{~g}, 1.05 \mathrm{mmol}, 2 \mathrm{eq})$ was added to a solution of methyl 2-(7-fluoro-1-oxoisoindolin-3yl)acetate $(0.170 \mathrm{~g}, 0.76 \mathrm{mmol})$ in anhydrous THF $(7.60 \mathrm{~mL})$ at $0{ }^{\circ} \mathrm{C}$ under a $\mathrm{N}_{2}$ atmosphere. The mixture was stirred at room temperature for $24 \mathrm{hr}$. The reaction was quenched with water $(20 \mathrm{~mL})$ and extracted with EtOAc (3 x $15 \mathrm{~mL})$, washed with brine, dried over $\mathrm{MgSO} 4$ and concentrated in vacuo to give the title compound as a white solid $(0.099 \mathrm{~g}, 67 \%)$. mp $135^{\circ} \mathrm{C} ;{ }^{1} \mathrm{H}$ NMR $\left(500 \mathrm{MHz}, \mathrm{CD}_{3} \mathrm{OD}\right) \delta 7.63(\mathrm{td}, J=7.9$, $4.9 \mathrm{~Hz}, 1 \mathrm{H}, \mathrm{ArH}), 7.38(\mathrm{~d}, J=7.6 \mathrm{~Hz}, 1 \mathrm{H}, \mathrm{ArH}), 7.15(\mathrm{t}, J=8.9 \mathrm{~Hz}, 1 \mathrm{H}, \mathrm{ArH}), 4.77(\mathrm{dt}, J=24.3,12.1 \mathrm{~Hz}$, $1 \mathrm{H}, \mathrm{ArCHN}), 3.85-3.66\left(\mathrm{~m}, 2 \mathrm{H}, \mathrm{CH}_{2} \mathrm{OH}\right), 2.31-2.13\left(\mathrm{~m}, 1 \mathrm{H}, \mathrm{CH}_{\mathrm{A}} \mathrm{H}_{\mathrm{B}} \mathrm{CH}_{2} \mathrm{OH}\right), 1.84-1.68(\mathrm{~m}, 1 \mathrm{H}$, $\left.\mathrm{CH}_{\mathrm{A}} \mathrm{H}_{\mathrm{B}} \mathrm{CH}_{2} \mathrm{OH}\right) ;{ }^{13} \mathrm{C} \mathrm{NMR}\left(126 \mathrm{MHz}, \mathrm{CD}_{3} \mathrm{OD}\right) \delta 169.7(\mathrm{~d}, \mathrm{~J}=2.2 \mathrm{~Hz}), 160.4\left(\mathrm{~d},{ }^{1} J_{\mathrm{C}-\mathrm{F}}=258.3 \mathrm{~Hz}\right), 152.6$ $\left(\mathrm{d},{ }^{3} J_{\mathrm{C}-\mathrm{F}}=2.6 \mathrm{~Hz}\right), 135.5\left(\mathrm{~d},{ }^{3} J_{\mathrm{C}-\mathrm{F}}=7.7 \mathrm{~Hz}\right), 120.2\left(\mathrm{~d},{ }^{4} J_{\mathrm{C}-\mathrm{F}}=4.2 \mathrm{~Hz}\right), 119.9\left(\mathrm{~d},{ }^{2} J_{\mathrm{C}-\mathrm{F}}=13.3 \mathrm{~Hz}\right), 116.2(\mathrm{~d}$, ${ }^{2} J_{\mathrm{C}-\mathrm{F}}=19.3 \mathrm{~Hz}$ ), 59.8, 56.0, 38.5; IR (thin film) $v_{\max }\left(\mathrm{cm}^{-1}\right): 3432.64(\mathrm{OH}), 1674.44(\mathrm{C}=\mathrm{O})$; HRMS (ESI): $\mathrm{m} / \mathrm{z}$ calculated for $\mathrm{C}_{10} \mathrm{H}_{10} \mathrm{O}_{2} \mathrm{FN}$ : 196.0696; for $[\mathrm{M}+\mathrm{H}]^{+}$found: 196.0754 ; for $[\mathrm{M}+\mathrm{Na}]^{+}$found: 218.0579 . 
<smiles>Cc1ccc(S(=O)(=O)OCCC2NC(=O)c3c(F)cccc32)cc1</smiles>

DMAP (0.006 g, $0.461 \mathrm{mmol}, 0.1 \mathrm{eq})$ and $\mathrm{Et}_{3} \mathrm{~N}(0.128 \mathrm{~mL}, 0.92 \mathrm{mmol}, 2 \mathrm{eq})$ were added to a solution of 7-fluoro-3-(2-hydroxyethyl)isoindolin-1-one $(0.090 \mathrm{~g}, 0.46 \mathrm{mmol})$ in a mixture of $\mathrm{CH}_{2} \mathrm{Cl}_{2}(2 \mathrm{~mL})$ and THF ( $2 \mathrm{~mL})$. The reaction mixture was then cooled to $0{ }^{\circ} \mathrm{C}$ under an $\mathrm{N}_{2}$ atmosphere, tosyl chloride $(0.097 \mathrm{~g}, 0.51$ mmol, $1.1 \mathrm{eq})$ added and the reaction mixture stirred at $\mathrm{rt}$ for $24 \mathrm{hr}$. Concentrated $\mathrm{HCl}$ was added $(3 \mathrm{~mL})$ and the mixture was extracted with EtOAc $(3 \times 10 \mathrm{~mL})$, washed with water $(10 \mathrm{~mL})$, brine $(10 \mathrm{~mL})$ and dried over $\mathrm{MgSO}_{4}$. Purification by column chromatography $\left(10 \% \mathrm{MeOH}\right.$ in $\left.\mathrm{CH}_{2} \mathrm{Cl}_{2}\right)$ afforded the title compound as a white solid $(0.113 \mathrm{~g}, 70 \%)$. mp $180{ }^{\circ} \mathrm{C} ;{ }^{1} \mathrm{H} \mathrm{NMR}\left(500 \mathrm{MHz}, \mathrm{CDCl}_{3}\right) \delta 7.79(\mathrm{~d}, J=8.3 \mathrm{~Hz}$, 2H, ArH), $7.56-7.51(\mathrm{~m}, 1 \mathrm{H}, \mathrm{ArH}), 7.37$ (d, J=8.0 Hz, 2H, ArH), 7.18 (d, J=7.6 Hz, 1H, ArH), 7.10 (m, $2 \mathrm{H}, \mathrm{ArH}), 4.75(\mathrm{dd}, J=8.7,3.7 \mathrm{~Hz}, 1 \mathrm{H}, \mathrm{ArCHN}), 4.32-4.11\left(\mathrm{~m}, 2 \mathrm{H}, \mathrm{CH}_{2} \mathrm{O}\right), 2.45\left(\mathrm{~s}, 3 \mathrm{H}, \mathrm{CH}_{3}\right), 2.42-$ $2.31\left(\mathrm{~m}, 1 \mathrm{H}, \mathrm{CH}_{\mathrm{A}} \mathrm{H}_{\mathrm{B}} \mathrm{CH}_{2} \mathrm{O}\right), 1.99-1.83\left(\mathrm{~m}, 1 \mathrm{H}, \mathrm{CH}_{\mathrm{A}} \mathrm{H}_{\mathrm{B}} \mathrm{CH}_{2} \mathrm{O}\right) ;{ }^{13} \mathrm{C} \mathrm{NMR}\left(126 \mathrm{MHz}, \mathrm{CDCl}_{3}\right) \delta 167.8$, $159.3\left(\mathrm{~d},{ }^{1} J_{\mathrm{C}-\mathrm{F}}=261.0 \mathrm{~Hz}\right), 149.4\left(\mathrm{~d},{ }^{3} J_{\mathrm{C}-\mathrm{F}}=2.8 \mathrm{~Hz}\right), 145.5,134.4\left(\mathrm{~d},{ }^{3} J_{\mathrm{C}-\mathrm{F}}=7.7 \mathrm{~Hz}\right), 132.6,130.2,128.1$, $119.0\left(\mathrm{~d},{ }^{2} J_{\mathrm{C}-\mathrm{F}}=13.4 \mathrm{~Hz}\right), 118.6\left(\mathrm{~d},{ }^{4} J_{\mathrm{C}-\mathrm{F}}=4.2 \mathrm{~Hz}\right), 116.0\left(\mathrm{~d},{ }^{2} J_{\mathrm{C}-\mathrm{F}}=19.5 \mathrm{~Hz}\right), 67.0,53.5,34.4,21.8 ; \mathrm{IR}$ (thin film) $v_{\max }\left(\mathrm{cm}^{-1}\right): 1707.76(\mathrm{C}=\mathrm{O}), 1354.82$ and $1178.83(\mathrm{~S}=\mathrm{O}) ;$ HRMS (ESI): m/z calculated for $\mathrm{C}_{17} \mathrm{H}_{16} \mathrm{FNO}_{4} \mathrm{~S}: 350.0784$; for $[\mathrm{M}+\mathrm{H}]^{+}$found: 350.0839 ; for $[\mathrm{M}+\mathrm{Na}]^{+}$found: 372.0658 . 
Synthesis of 3-\{2-[4-(3,4-dimethylphenyl)piperazin-1-yl]ethyl\}-7-fluoro-2,3-dihydro-1H-isoindol-1one 22

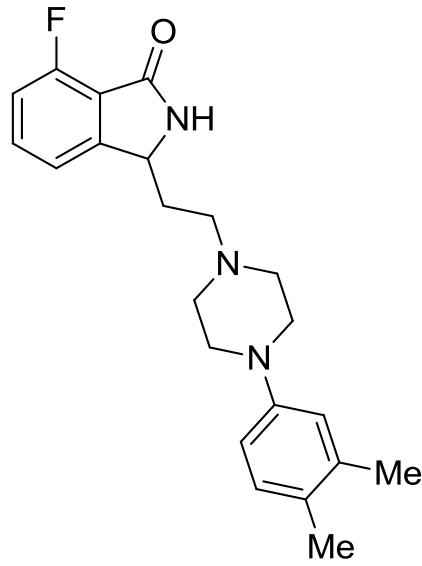

$\mathrm{K}_{2} \mathrm{CO}_{3}(0.214 \mathrm{~g}, 1.55 \mathrm{mmol}, 5 \mathrm{eq})$ was added to a solution of 2-(4-fluoro-3-oxo-2,3-dihydro-1H-isoindol1-yl)ethyl 4-methylbenzene-1-sulfonate $(0.110 \mathrm{~g}, 0.31 \mathrm{mmol}, 1 \mathrm{eq})$ and 1-(3,4-dimethylphenyl)-piperazine, $(0.068 \mathrm{~g}, 0.35 \mathrm{mmol}, 1.1 \mathrm{eq})$ in $\mathrm{CH}_{3} \mathrm{CN}(10 \mathrm{~mL})$ and the mixture heated at $65^{\circ} \mathrm{C}$ under $\mathrm{N}_{2}$ overnight. After cooling to rt, the solvent was evaporated, and the residue was stirred in $10 \mathrm{~mL}$ of water for $1 \mathrm{hr}$. The water was decanted and the solid was disolved in EtOAc and dried over $\mathrm{MgSO}_{4}$. Purification by column chromatography (5\% MeOH in DCM) afforded the title compound as a white solid $(0.079 \mathrm{~g}, 69 \%)$. $\mathrm{mp} 92-$ $95^{\circ} \mathrm{C} ;{ }^{1} \mathrm{H}$ NMR (500 MHz, $\left.\mathrm{CDCl}_{3}\right) \delta 7.81(\mathrm{~s}, 1 \mathrm{H}, \mathrm{ArH}), 7.57-7.48(\mathrm{~m}, 1 \mathrm{H}, \mathrm{ArH}), 7.22(\mathrm{t}, J=7.1 \mathrm{~Hz}, 1 \mathrm{H}$, ArH), $7.08(\mathrm{t}, J=8.7 \mathrm{~Hz}, 1 \mathrm{H}, \mathrm{ArH}), 7.01(\mathrm{~d}, J=8.2 \mathrm{~Hz}, 1 \mathrm{H}, \mathrm{ArH}), 6.74(\mathrm{~d}, J=2.5 \mathrm{~Hz}, 1 \mathrm{H}, \mathrm{ArH}), 6.68(\mathrm{dd}$, $J=8.2,2.6 \mathrm{~Hz}, 1 \mathrm{H}, \mathrm{ArH}), 4.66(\mathrm{dd}, J=9.6,3.2 \mathrm{~Hz}, 1 \mathrm{H}, \mathrm{ArCHN}), 3.26-3.10\left(\mathrm{~m}, 4 \mathrm{H}, \mathrm{CH}_{2} \mathrm{NAr}\right), 2.75-$ $2.67\left(\mathrm{~m}, 2 \mathrm{H}, \mathrm{CH}_{2} \mathrm{~N}\right), 2.67-2.60\left(\mathrm{~m}, 2 \mathrm{H}, \mathrm{CH}_{2} \mathrm{~N}\right), 2.60-2.54\left(\mathrm{~m}, 2 \mathrm{H}, \mathrm{CH}_{2} \mathrm{~N}\right), 2.22\left(\mathrm{~s}, 3 \mathrm{H}, \mathrm{CH}_{3}\right), 2.18(\mathrm{~s}$, $\left.3 \mathrm{H}, \mathrm{CH}_{3}\right), 2.17-2.11\left(\mathrm{~m}, 1 \mathrm{H}, \mathrm{CH}_{\mathrm{A}} \mathrm{H}_{\mathrm{B}} \mathrm{CH}_{2} \mathrm{~N}\right), 1.86-1.71\left(\mathrm{~m}, 1 \mathrm{H}, \mathrm{CH}_{\mathrm{A}} \mathrm{H}_{\mathrm{B}} \mathrm{CH}_{2} \mathrm{~N}\right) ;{ }^{13} \mathrm{C} \mathrm{NMR}(126 \mathrm{MHz}$, $\left.\mathrm{CDCl}_{3}\right) \delta 167.6,159.3\left(\mathrm{~d},{ }^{1} J_{\mathrm{C}-\mathrm{F}}=260.3 \mathrm{~Hz}\right), 150.6\left(\mathrm{~d},{ }^{3} J_{\mathrm{C}-\mathrm{F}}=2.6 \mathrm{~Hz}\right), 149.5,137.2,133.9\left(\mathrm{~d},{ }^{3} J_{\mathrm{C}-\mathrm{F}}=7.6\right.$ $\mathrm{Hz}), 130.2,128.3,119.4\left(\mathrm{~d},{ }^{2} J_{\mathrm{C}-\mathrm{F}}=13.1 \mathrm{~Hz}\right), 118.4\left(\mathrm{~d},{ }^{4} J_{\mathrm{C}-\mathrm{F}}=4.1 \mathrm{~Hz}\right), 118.3,115.5\left(\mathrm{~d},{ }^{2} J_{\mathrm{C}-\mathrm{F}}=19.5 \mathrm{~Hz}\right)$, 114.1, 56.8, 56.2, 53.4, 49.8, 31.5, 20.3, 18.9; IR (thin film) $v_{\max }\left(\mathrm{cm}^{-1}\right): 1706.83(\mathrm{C}=\mathrm{O}), 1238.83(\mathrm{C}-\mathrm{N})$; HRMS (ESI): $\mathrm{m} / \mathrm{z}$ calculated for $\mathrm{C}_{22} \mathrm{H}_{26} \mathrm{FN}_{3} \mathrm{O}: 368.2060$; for $[\mathrm{M}+\mathrm{H}]^{+}$found: 368.2133 ; for $[\mathrm{M}+\mathrm{Na}]^{+}$found: 390.1929 . 
Methyl 2-(2-hydroxy-3-oxoisoindolin-1-yl)acetate 4a

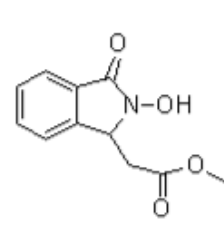

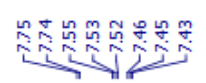

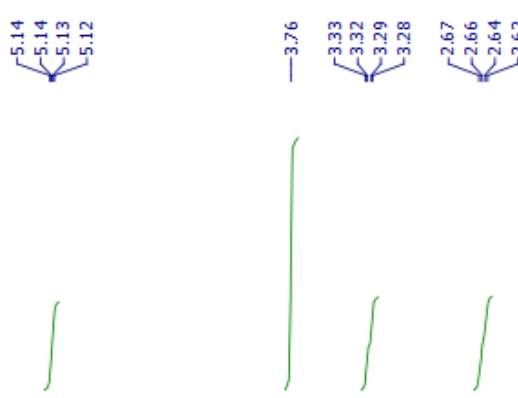

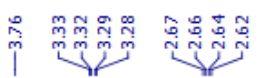

$500 \mathrm{MHz}_{\mathrm{CDCl}}$
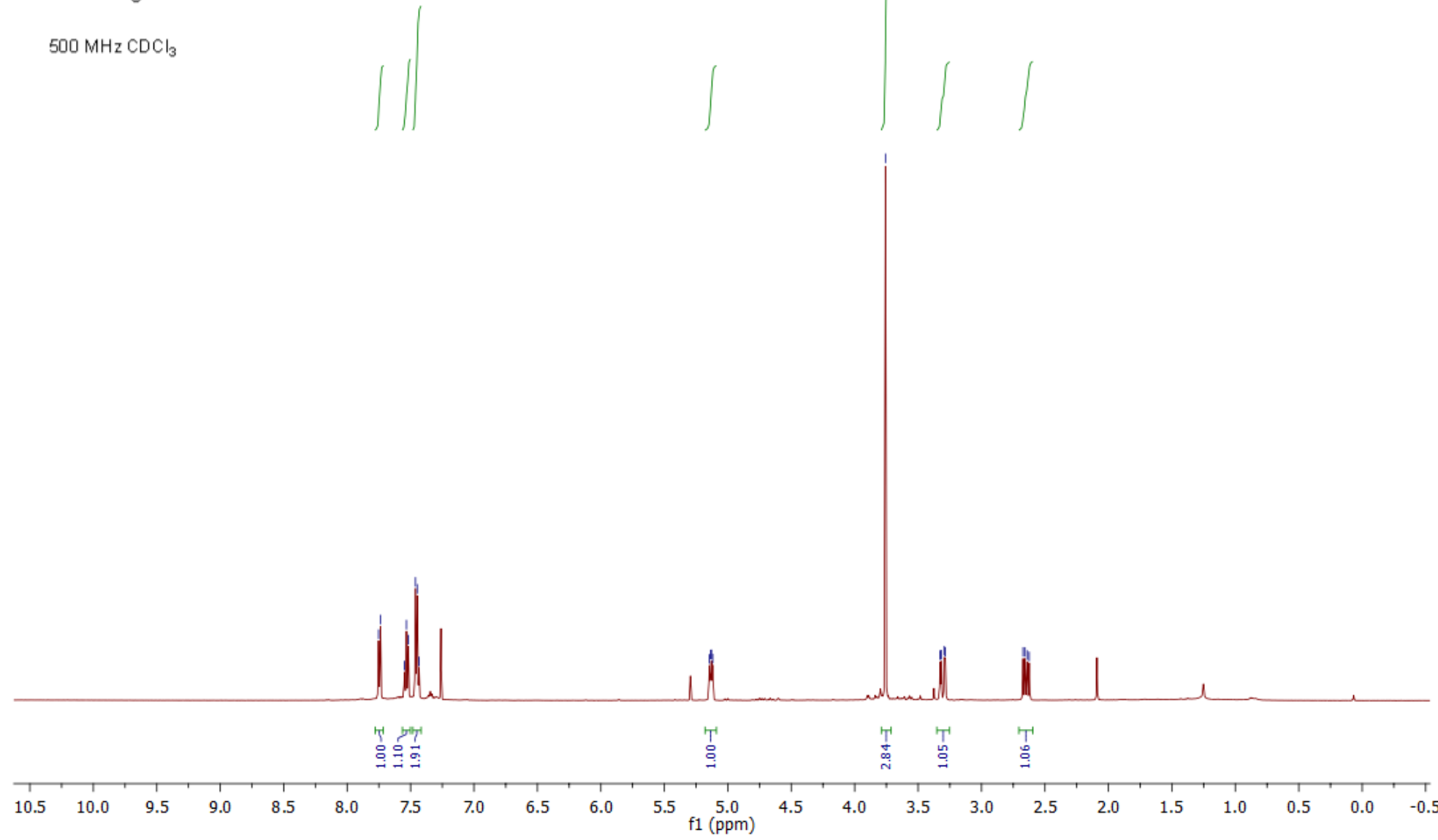
Methyl 2-(2-hydroxy-3-oxoisoindolin-1-yl)acetate 4a

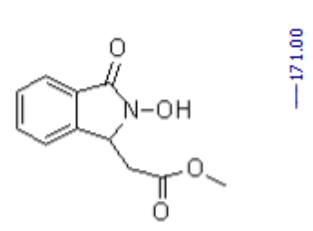

$75 \mathrm{MHz} C D C l_{3}$

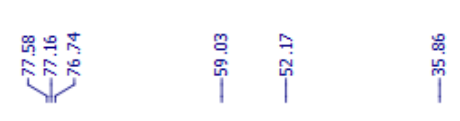

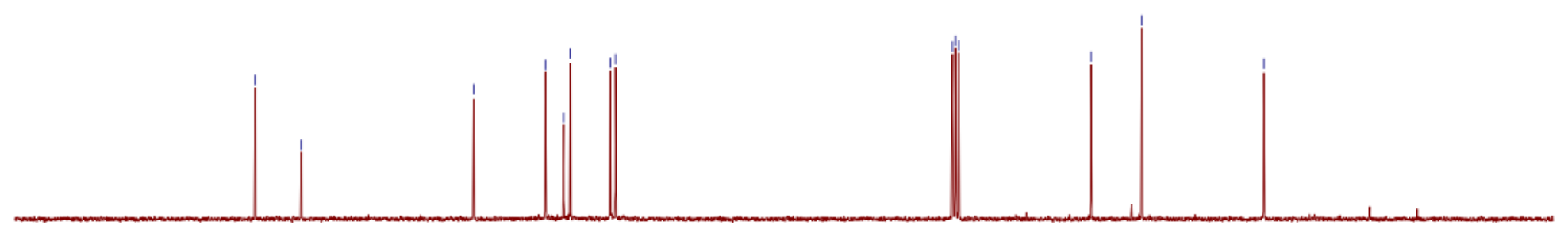

200 $190 \quad 180$ $170 \quad 160$ 150 140 $130 \quad 120$ $110 \stackrel{100}{10}$ 100
$\mathrm{f} 1(\mathrm{ppm})$ \begin{tabular}{ll|l}
\hline & 1 \\
\hline & 80
\end{tabular} 70 50 40 \begin{tabular}{ll|l}
1 & 1 & 1 \\
20 & 10 & 0
\end{tabular} 
Methyl 2-(6-hydroxy-7-oxo-6,7-dihydro-5H-[1,3]dioxolo[4,5-f]isoindol-5-yl)acetate 4b

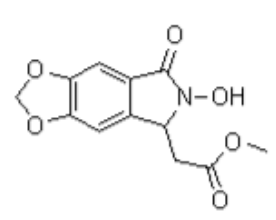

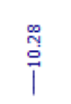

$300 \mathrm{MHz} \mathrm{CDCl}_{3}$

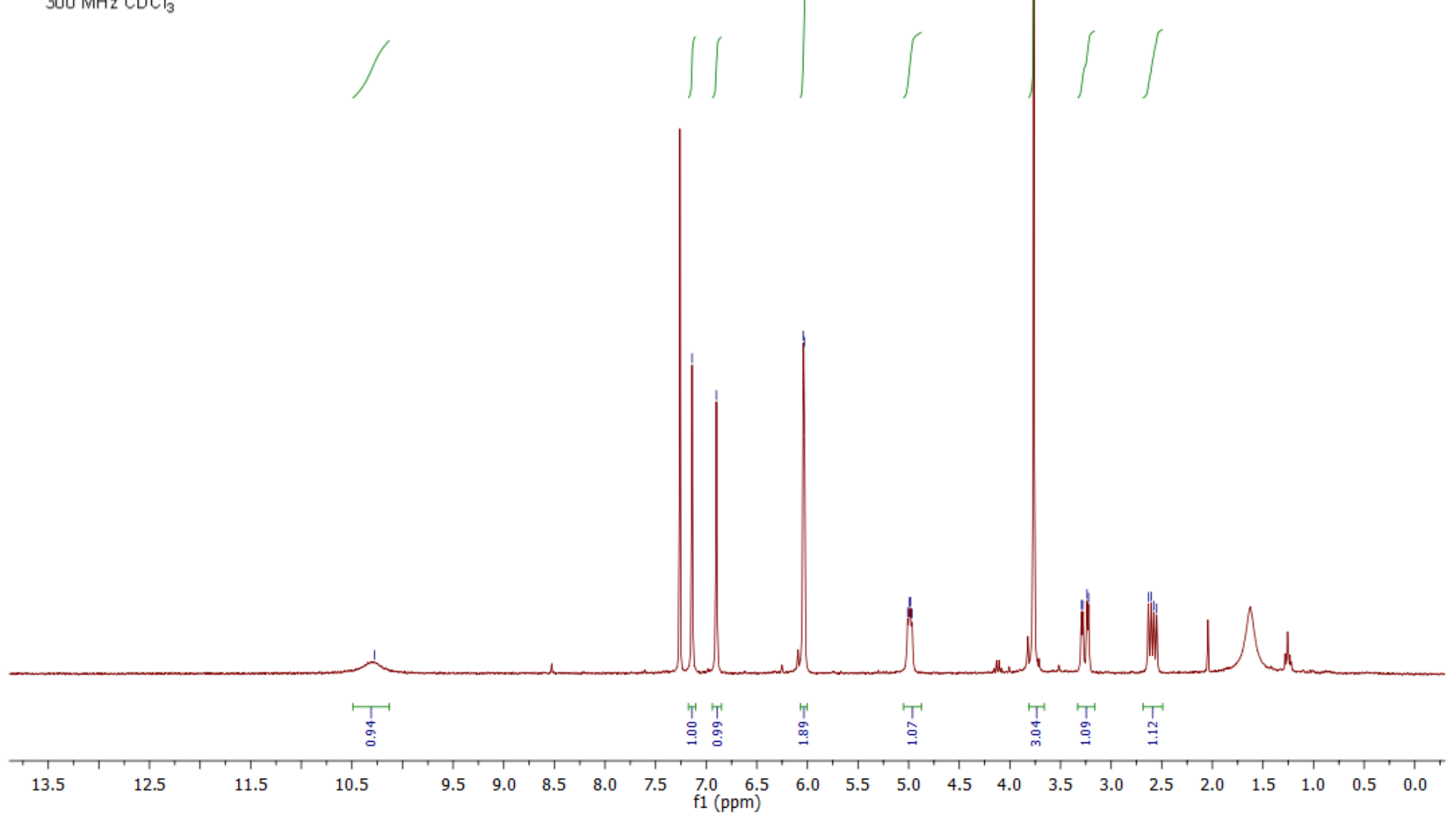


32

Methyl 2-(6-hydroxy-7-oxo-6,7-dihydro-5H-[1,3]dioxolo[4,5-f]isoindol-5-yl)acetate 4b

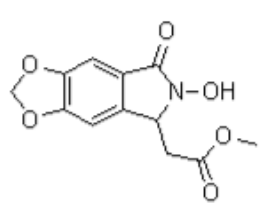

$75 \mathrm{MHz} \mathrm{CDCl}_{3}$

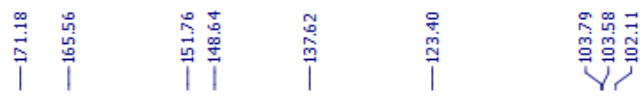

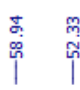

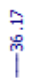

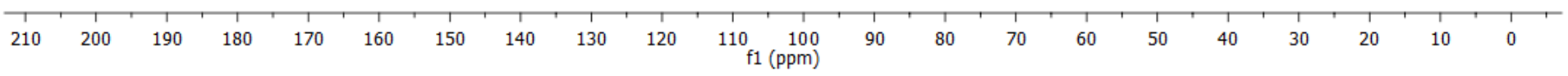


Methyl 2-(2-hydroxy-3-oxo-5-(trifluoromethyl)isoindolin-1-yl)acetate 4c

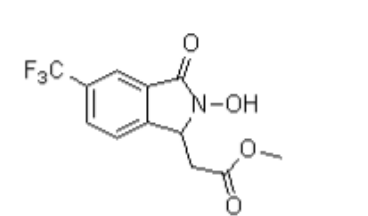

$500 \mathrm{MHz} \mathrm{CDCl}_{3}$
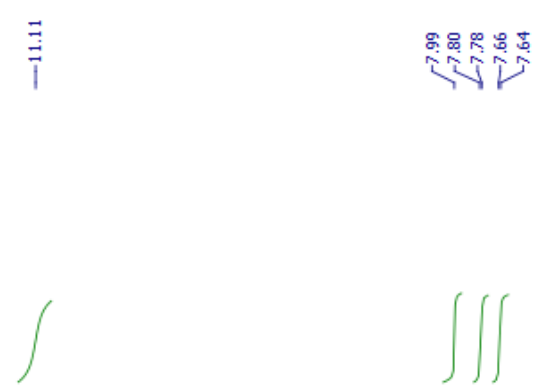

죽줄웡
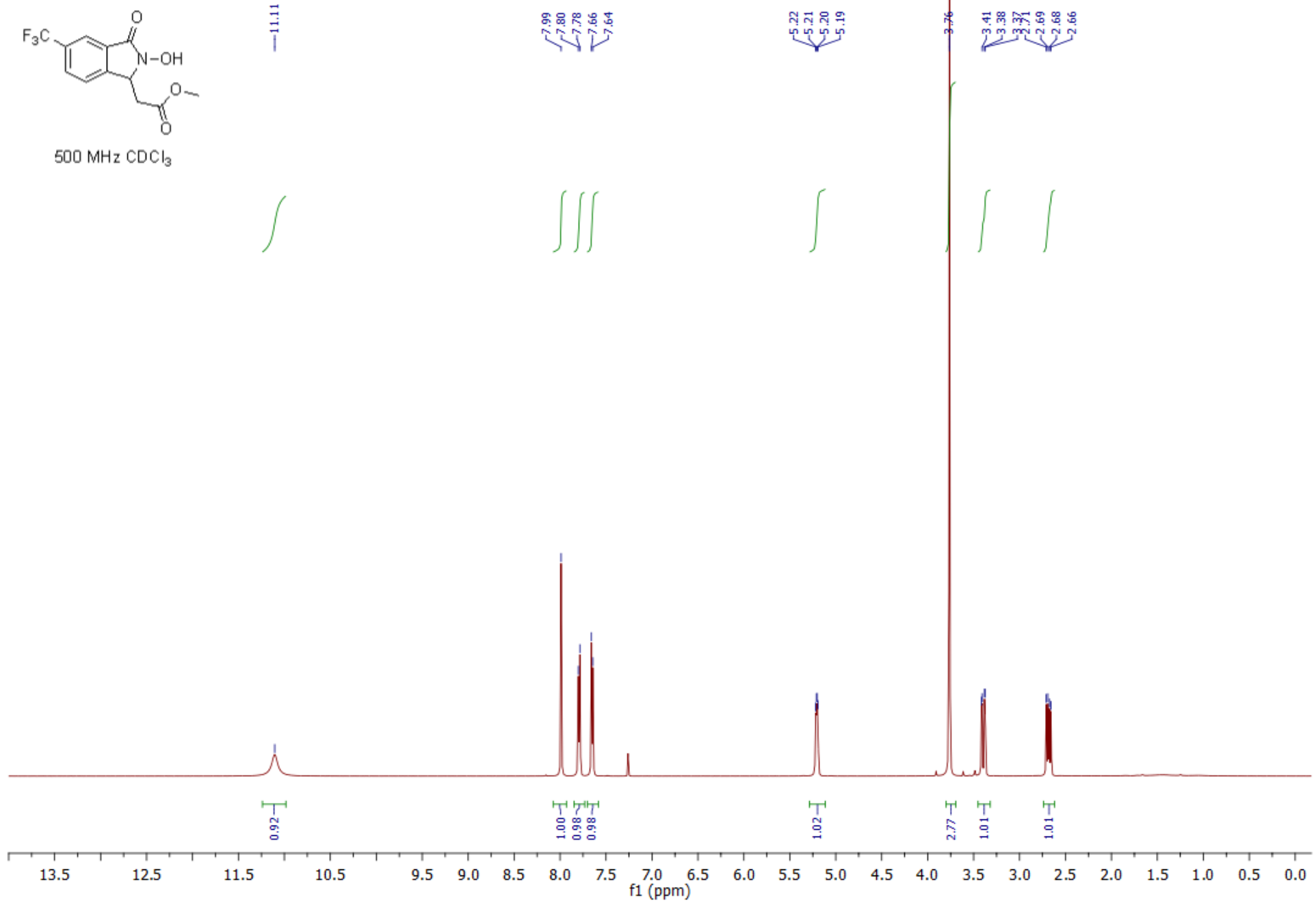
Methyl 2-(2-hydroxy-3-oxo-5-(trifluoromethyl)isoindolin-1-yl)acetate 4c

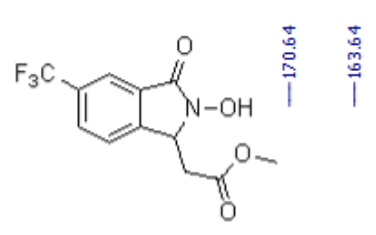

$126 \mathrm{MHz} \mathrm{CDCl}$

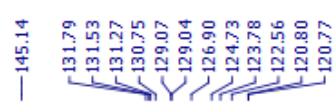

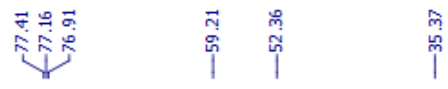

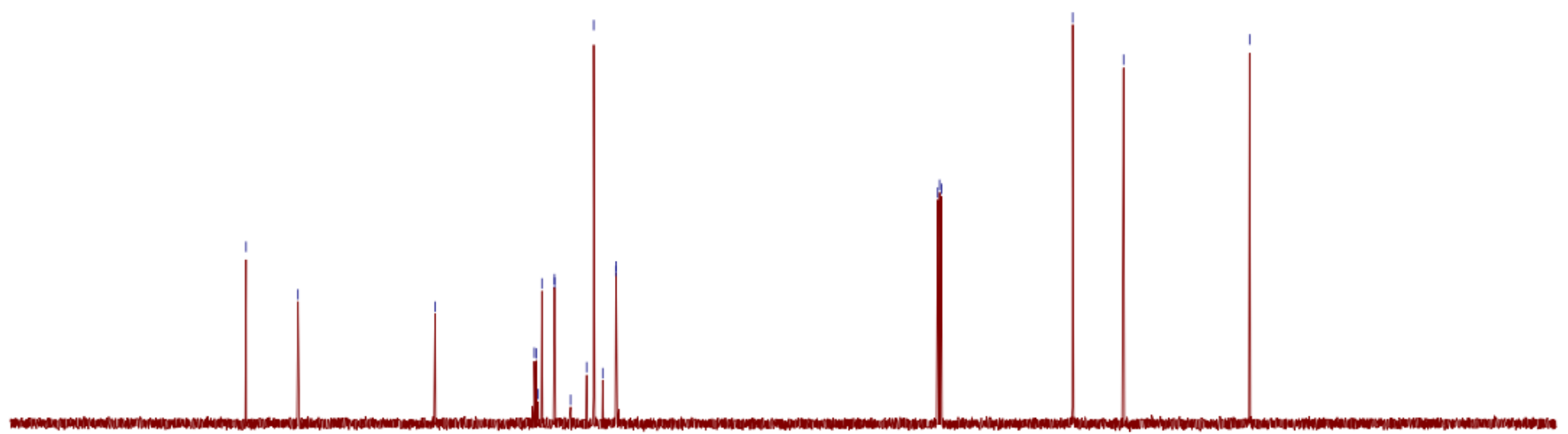

200
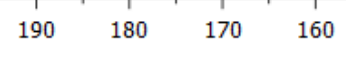

150
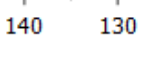

110
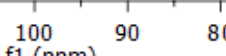

$80 \quad 70$

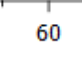

$60 \quad 50$

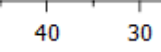


Methyl 2-(2-hydroxy-3-oxo-5-fluoroisoindolin-1-yl)acetate 4d

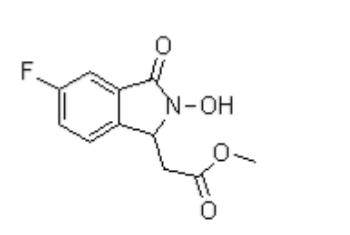

$300 \mathrm{MHz} \mathrm{CDCl}_{3}$
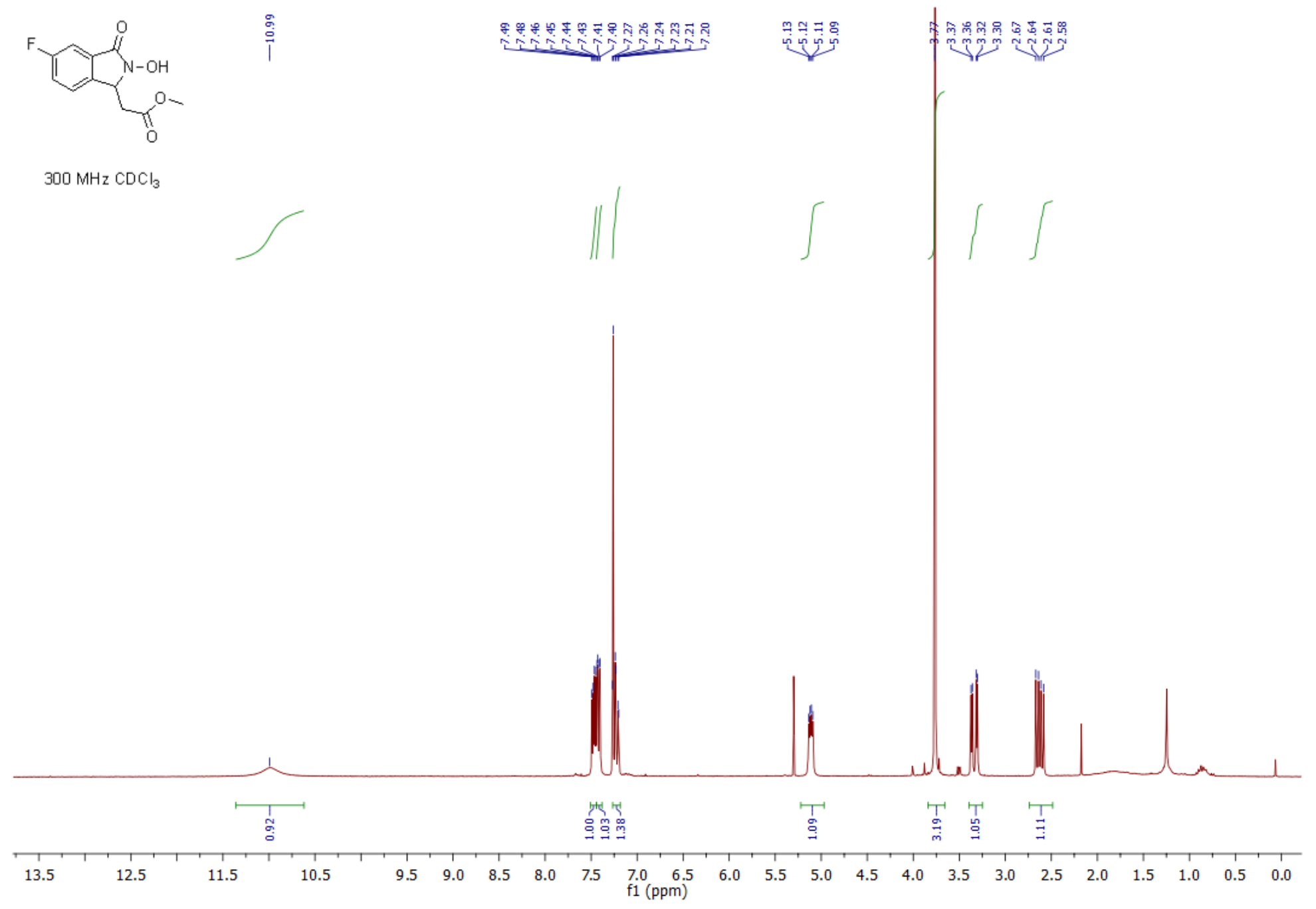

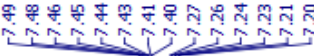

यू⿻ 
Methyl 2-(2-hydroxy-3-oxo-5-fluoroisoindolin-1-yl)acetate 4d
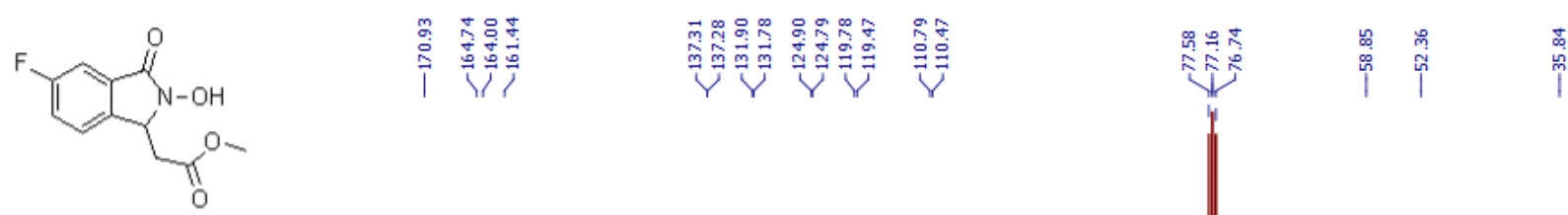

$75 \mathrm{MHz} \mathrm{CDCl}_{3}$

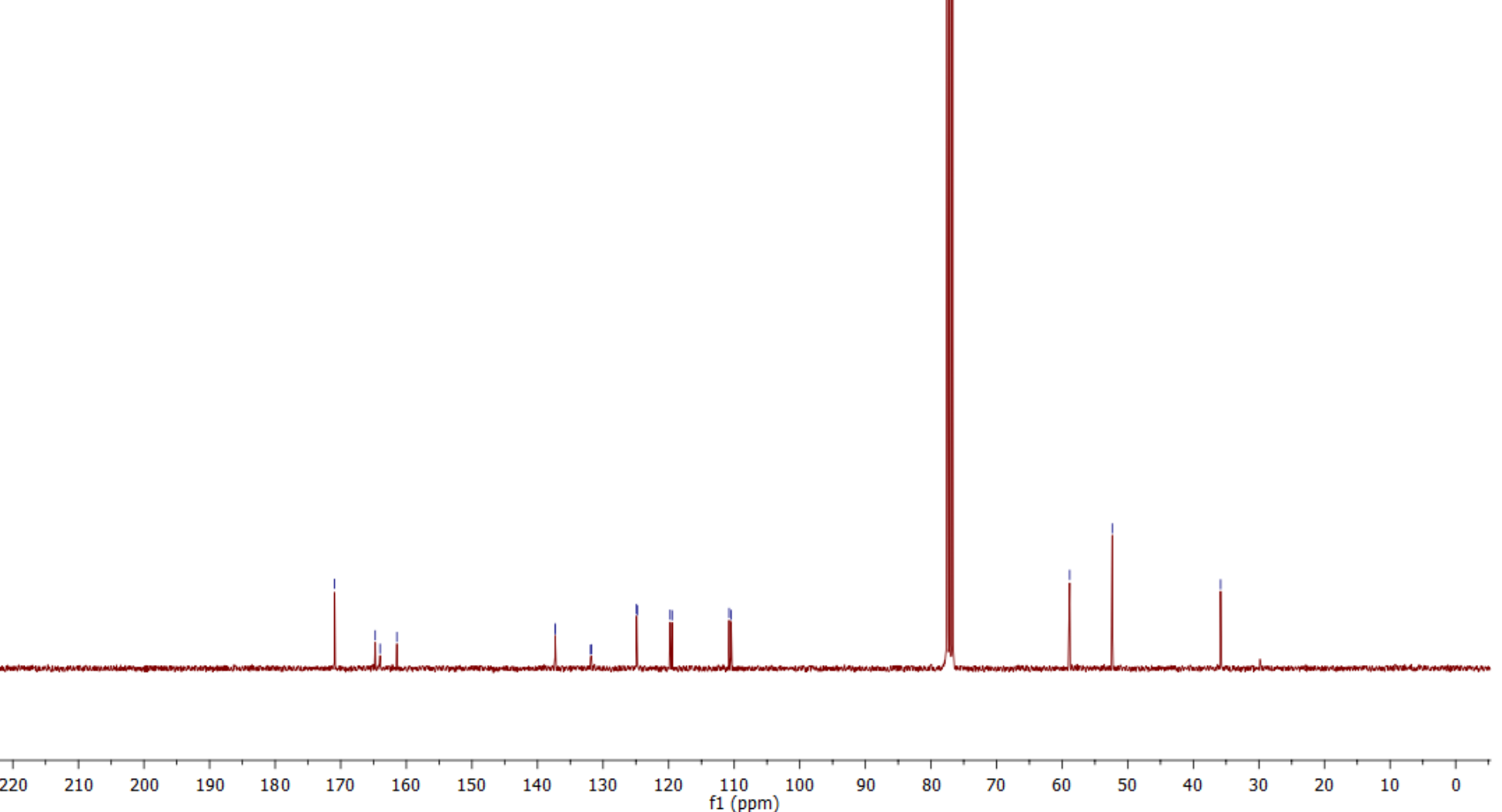


Methyl 2-(2-hydroxy-5,6-dimethoxy-3-oxo-2,3-dihydro-1H-isoindol-1-yl)acetate 4e

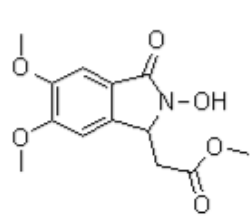

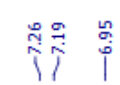

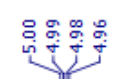

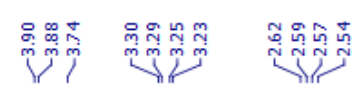

$300 \mathrm{MHz}_{2} \mathrm{CDCl}_{3}$
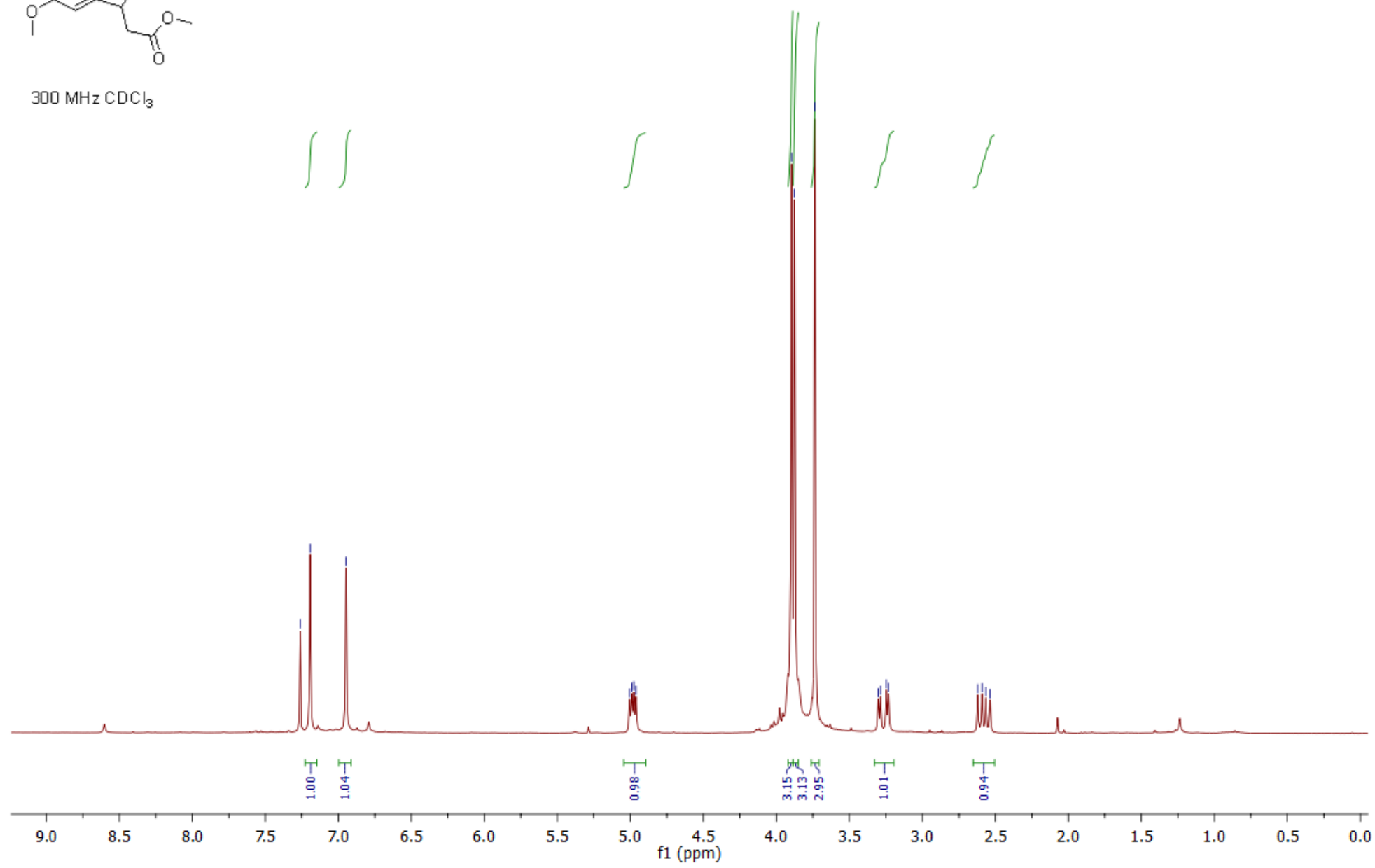
Methyl 2-(2-hydroxy-5,6-dimethoxy-3-oxo-2,3-dihydro-1H-isoindol-1-yl)acetate 4e

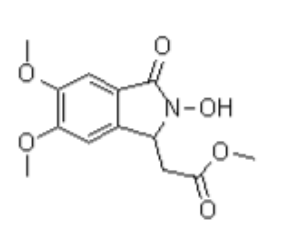

il

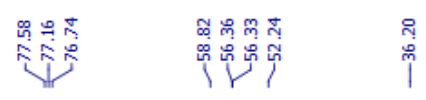

$75 \mathrm{MHz} C D C l_{3}$
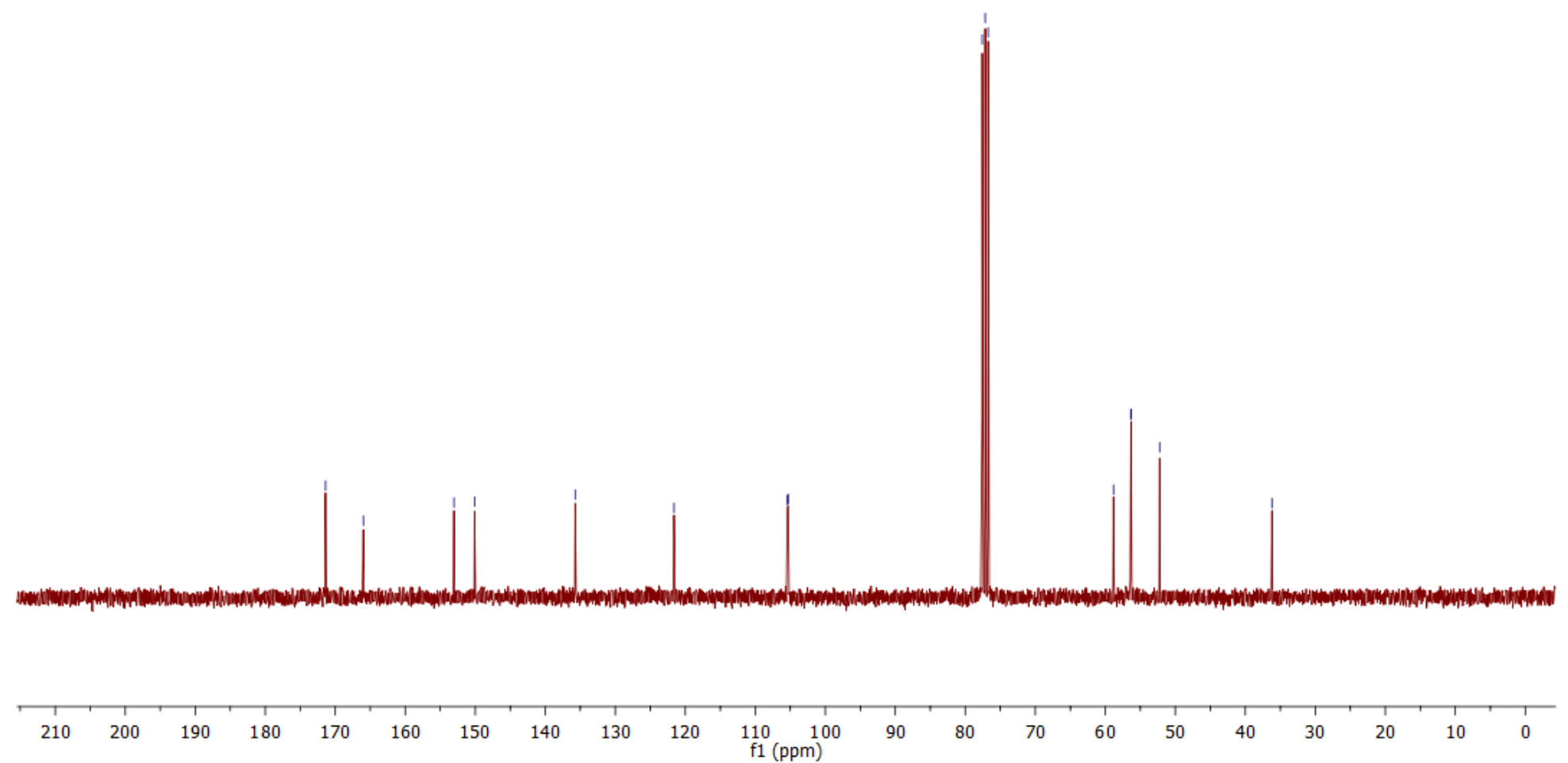
Methyl 2-(2-hydroxy-5-methoxy-3-oxoisoindolin-1-yl)acetate 4f
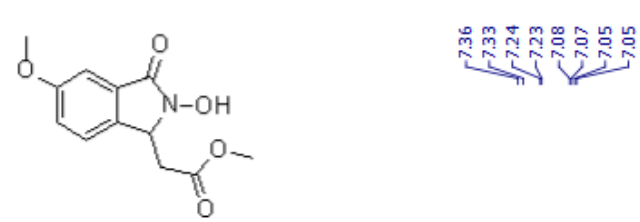

$300 \mathrm{MHz} \mathrm{CDCl}_{3}$

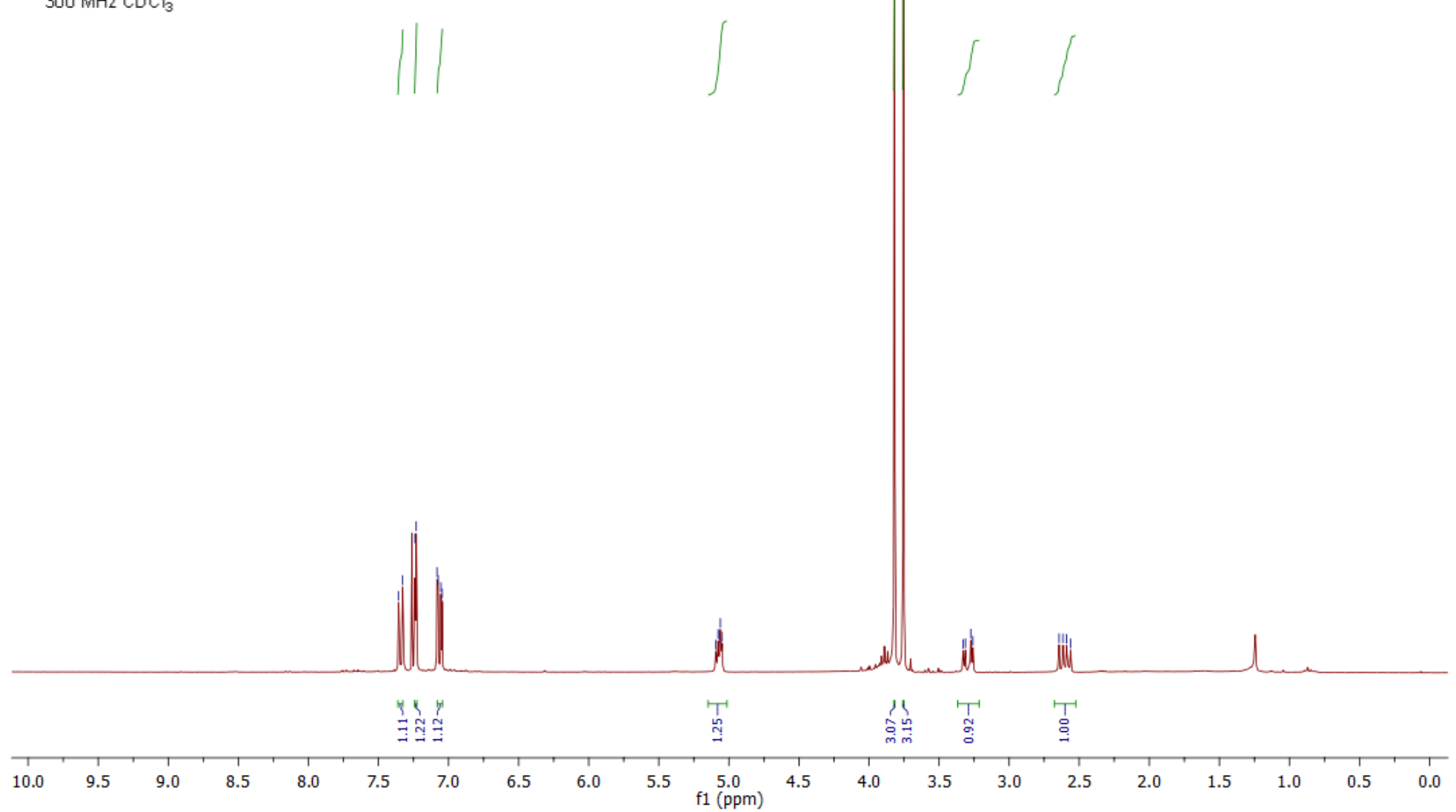


Methyl 2-(2-hydroxy-5-methoxy-3-oxoisoindolin-1-yl)acetate 4 f

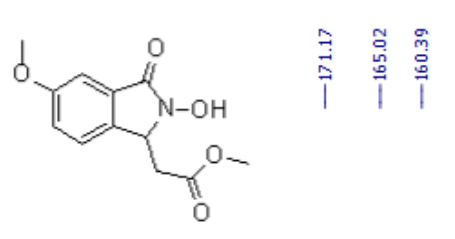

$75 \mathrm{MHz} \mathrm{CDCl}_{3}$

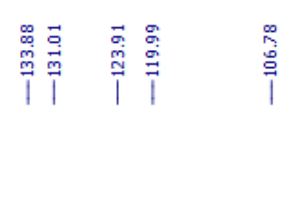

$\stackrel{\infty}{0}$

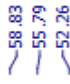

管 
Methyl 2-(2-hydroxy-3-oxo-4-fluoroisoindolin-1-yl)acetate 4g

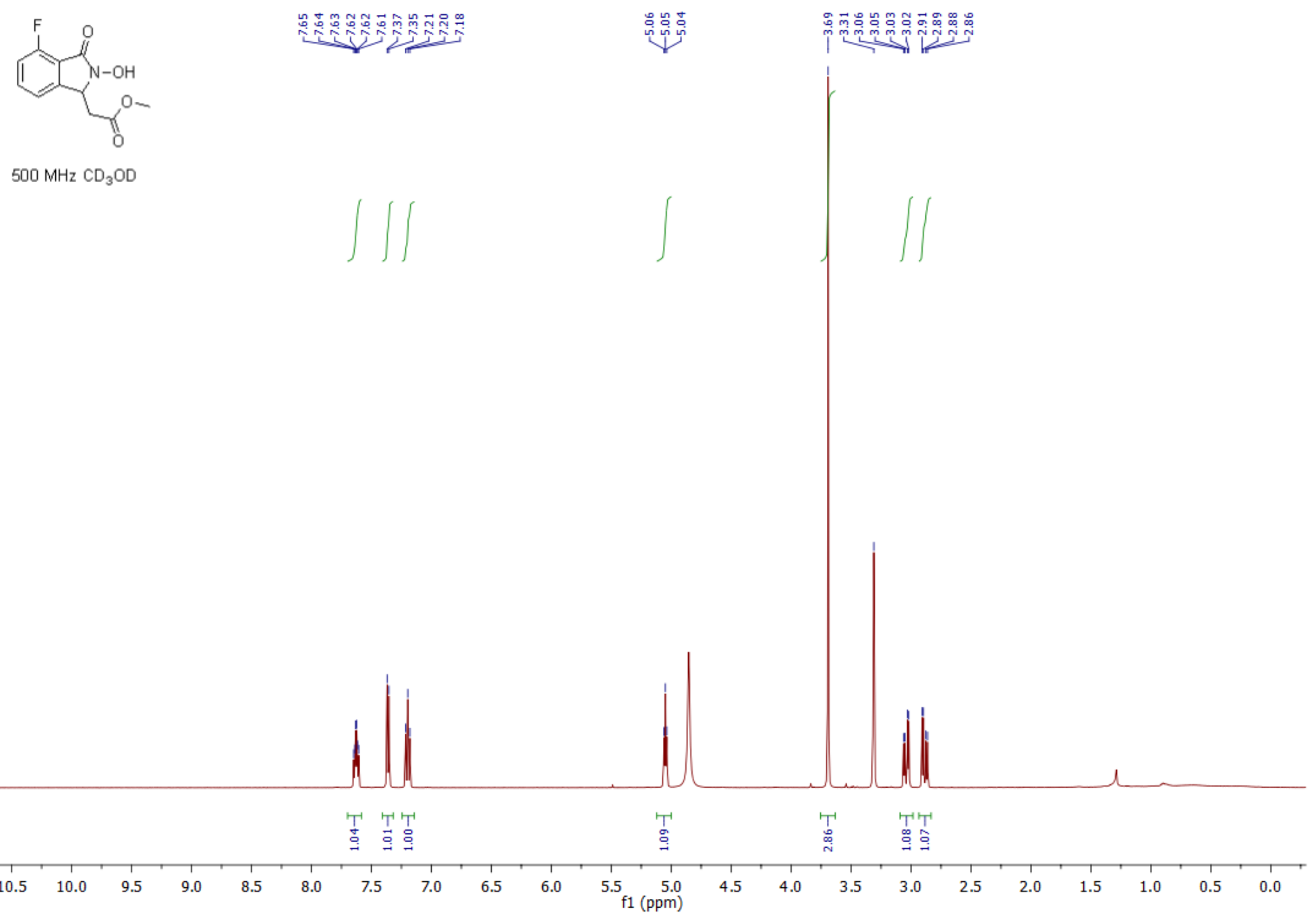


Methyl 2-(2-hydroxy-3-oxo-4-fluoroisoindolin-1-yl)acetate 4g
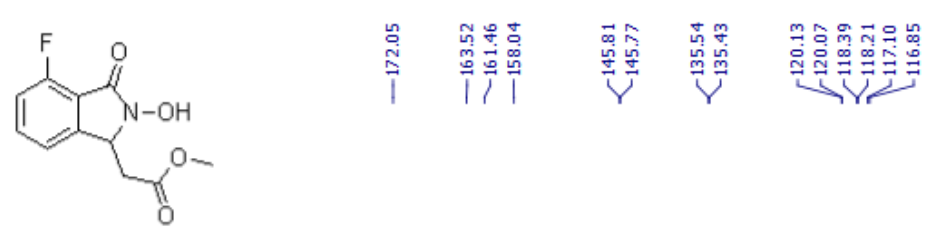

$75 \mathrm{MHz} \mathrm{CD}_{3} \mathrm{OD}$

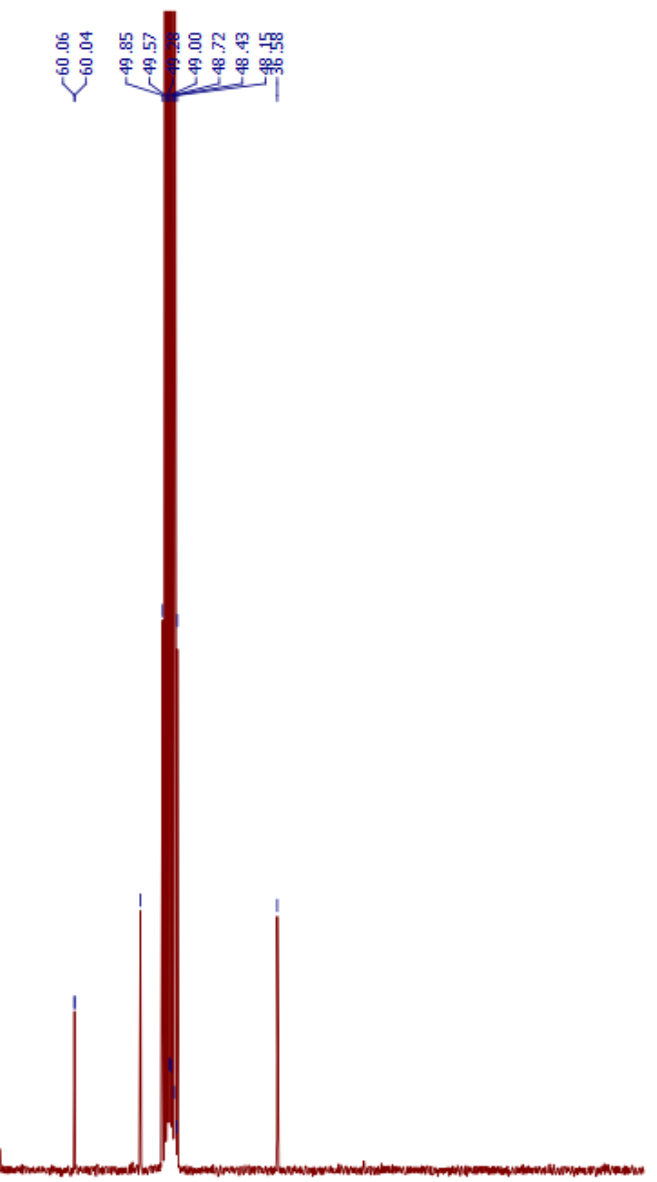

$\begin{array}{lllllllllll}210 & 200 & 190 & 180 & 170 & 160 & 150 & 140 & 130 & 120 & 110 \\ \mathrm{f} 1(\mathrm{ppm})\end{array}$ 
2-(2-hydroxy-3-oxo-5-(trifluoromethyl)isoindolin-1-yl)-N-phenylacetamide $14300 \mathrm{MHz}$ (DMSO)

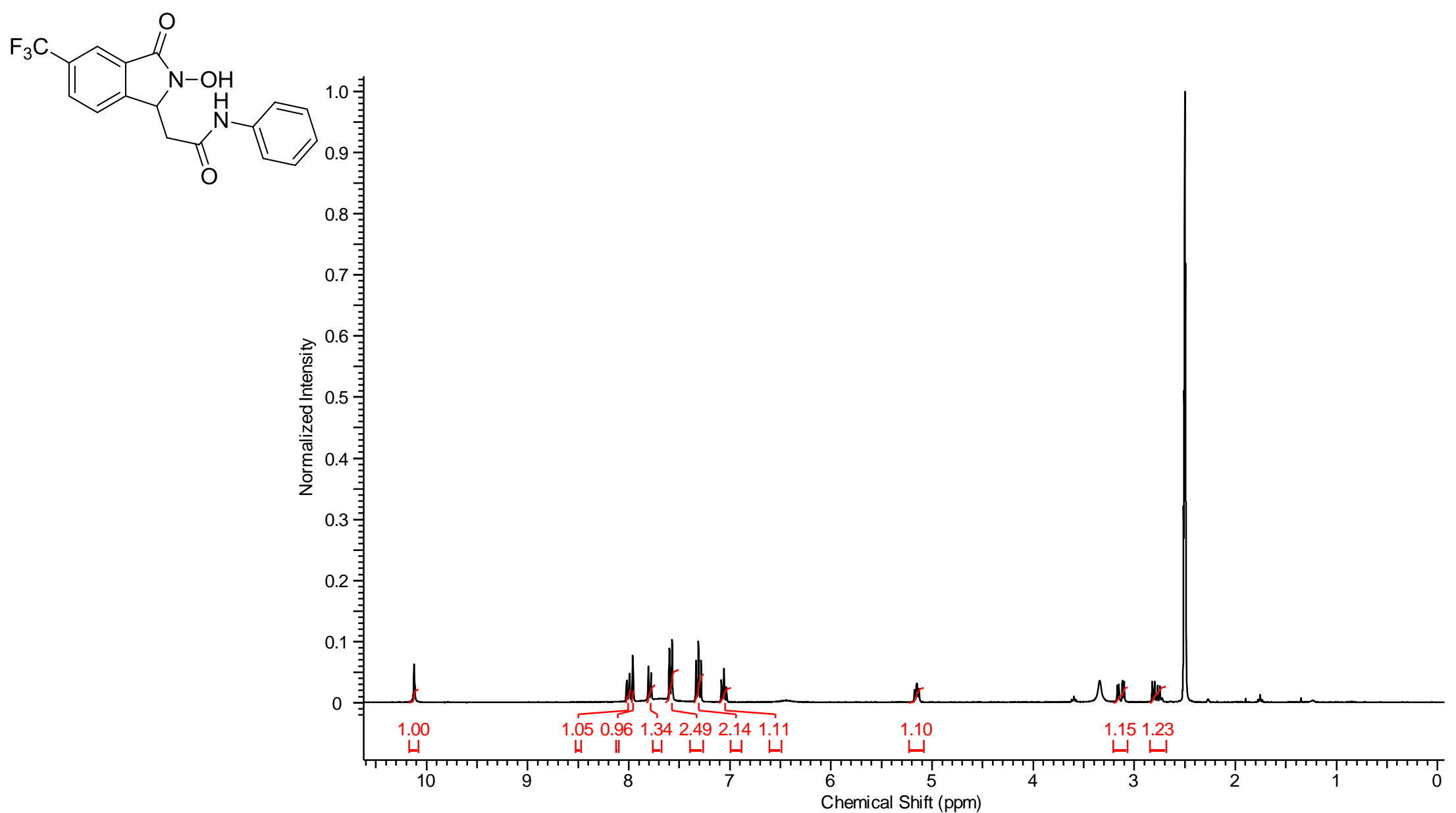


2-(2-hydroxy-3-oxo-5-(trifluoromethyl)isoindolin-1-yl)-N-phenylacetamide $14126 \mathrm{MHz}$ (DMSO)

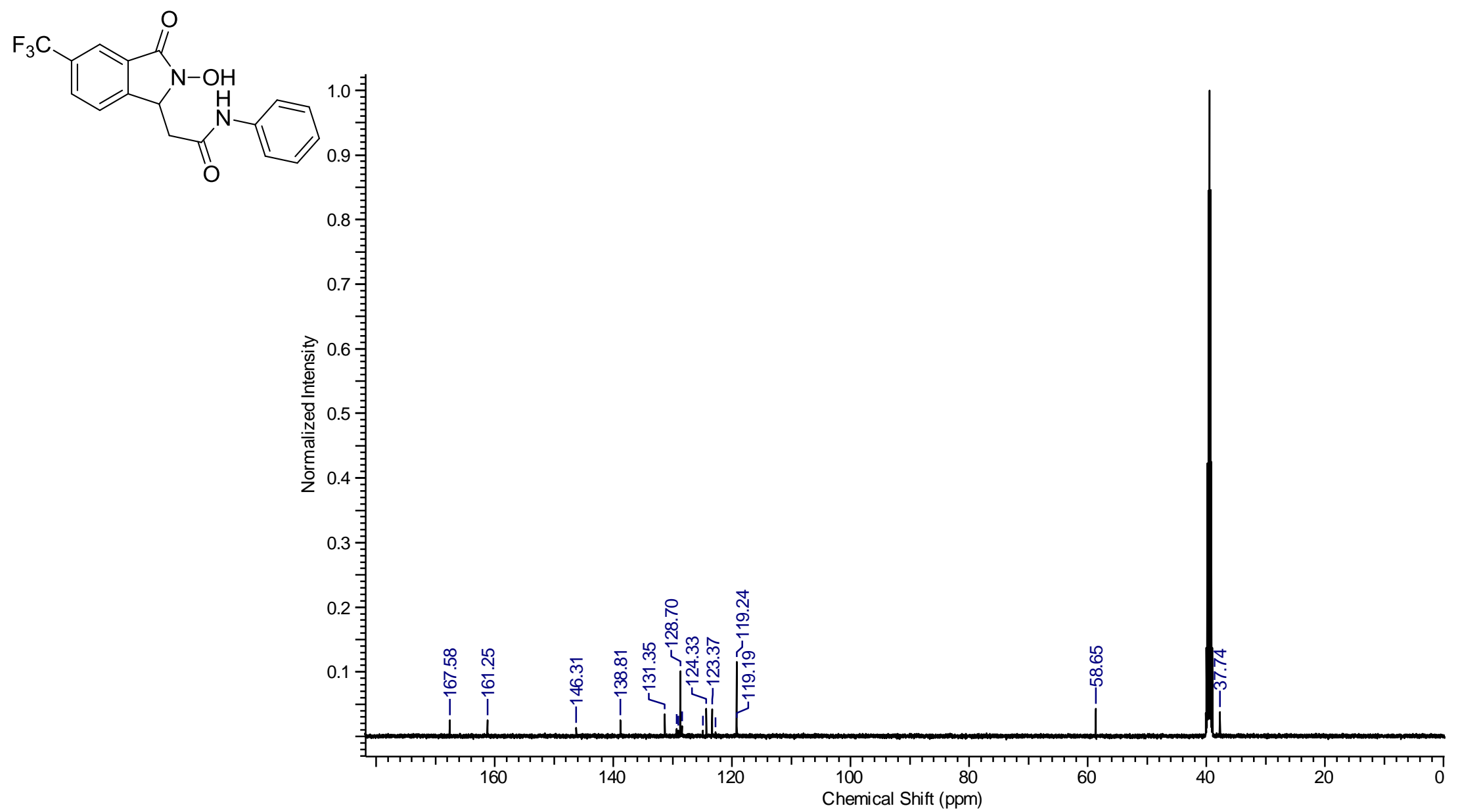


Ethyl 2-(3-oxoisoindolin-1-yl)acetate 15a $300 \mathrm{MHz}$ (MeOD)

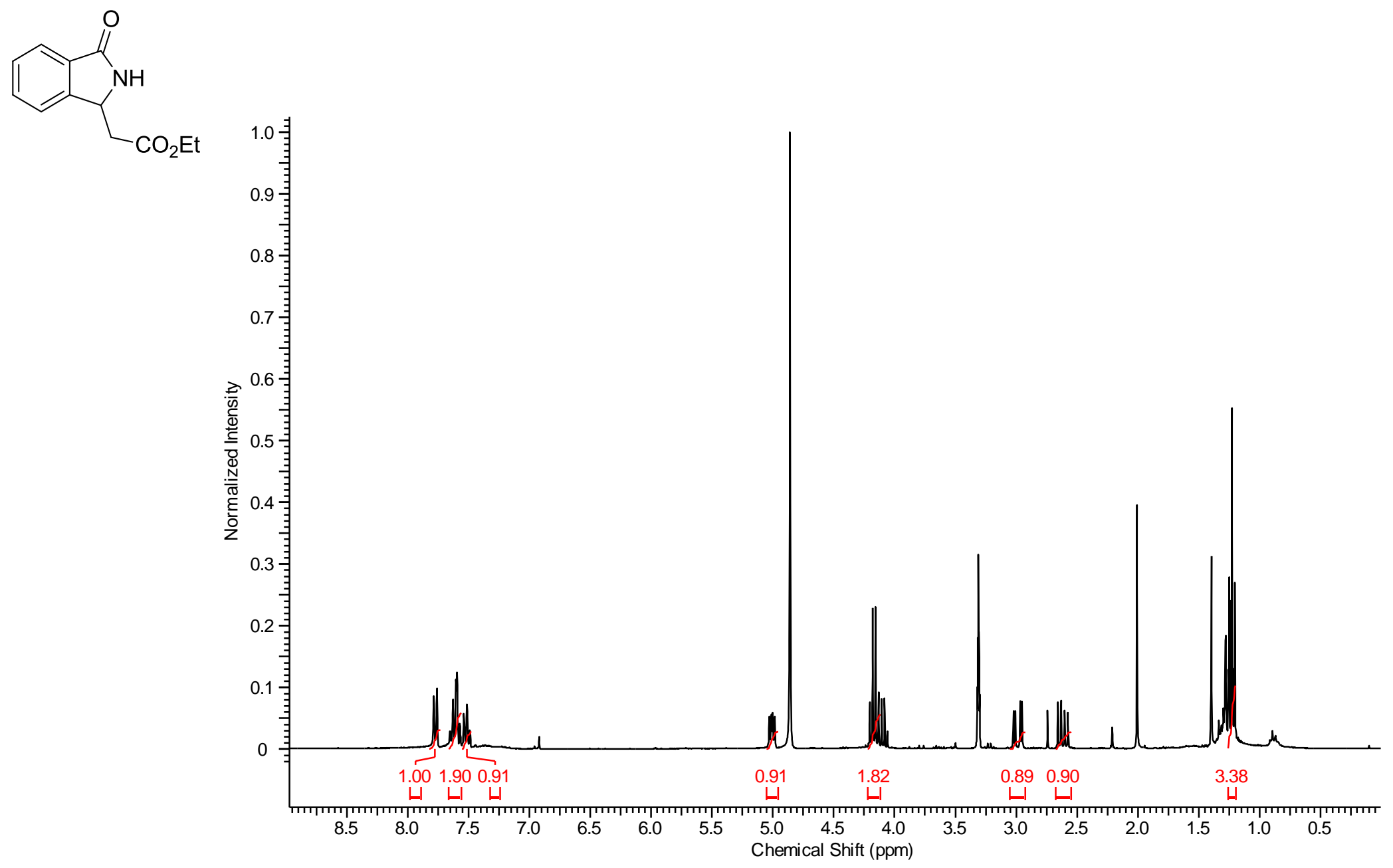


Ethyl 2-(3-oxoisoindolin-1-yl)acetate 15a 75.5 MHz (MeOD)

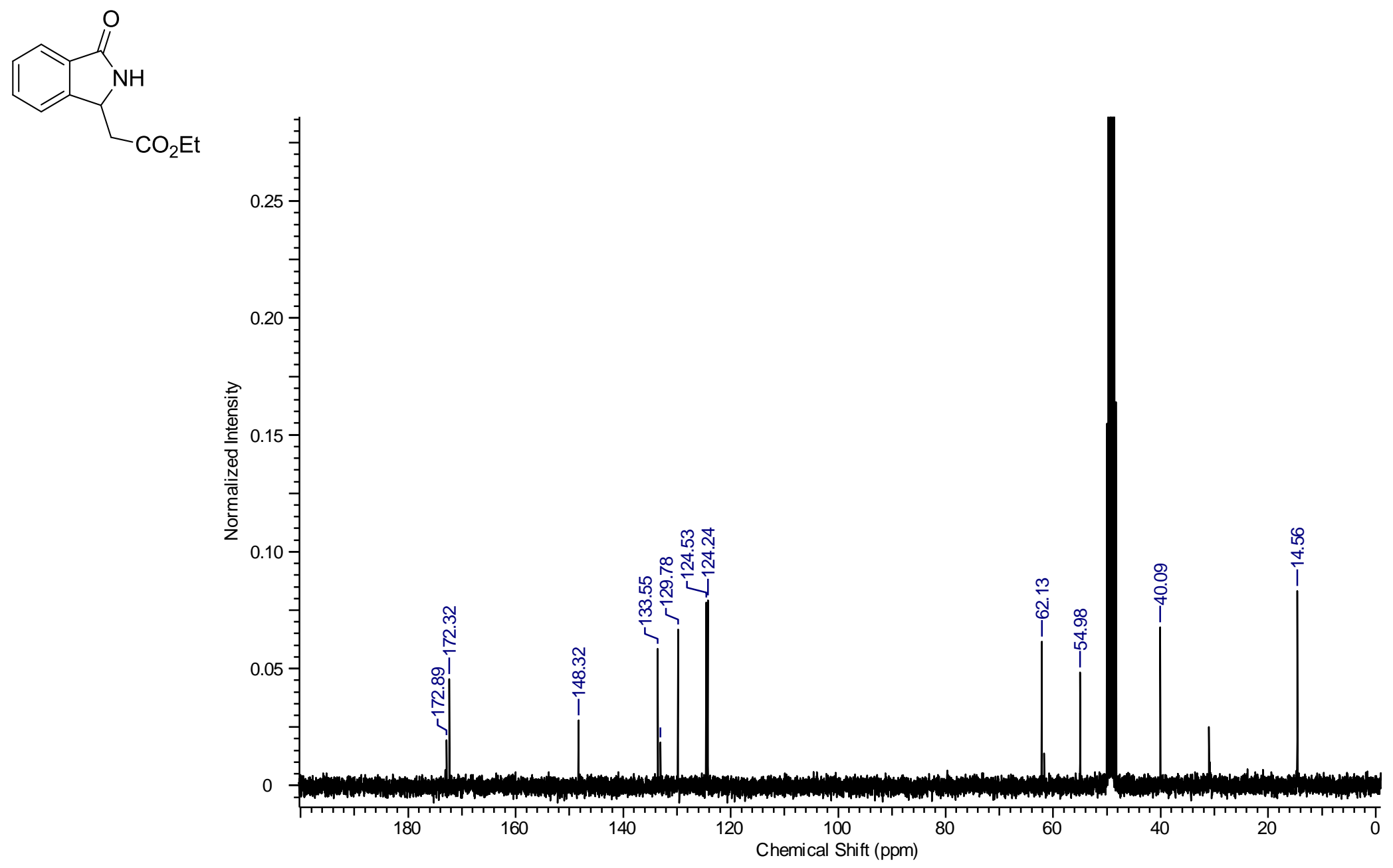


Ethyl 2-(7-oxo-6,7-dihydro-5H-[1,3]dioxolo[4,5-f]isoindol-5-yl)acetate 15b

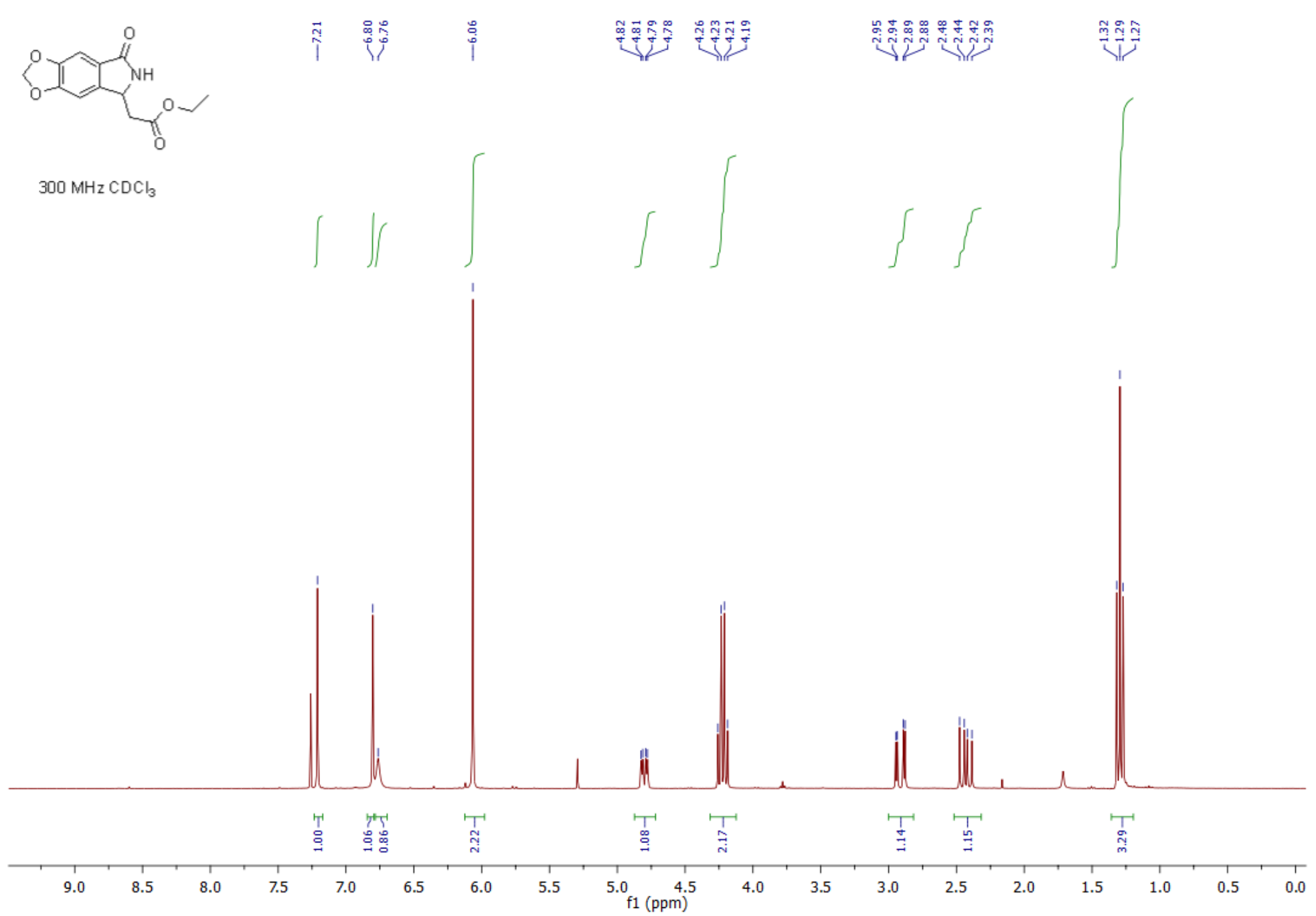


Ethyl 2-(7-oxo-6,7-dihydro-5H-[1,3]dioxolo[4,5-f]isoindol-5-yl)acetate 15b

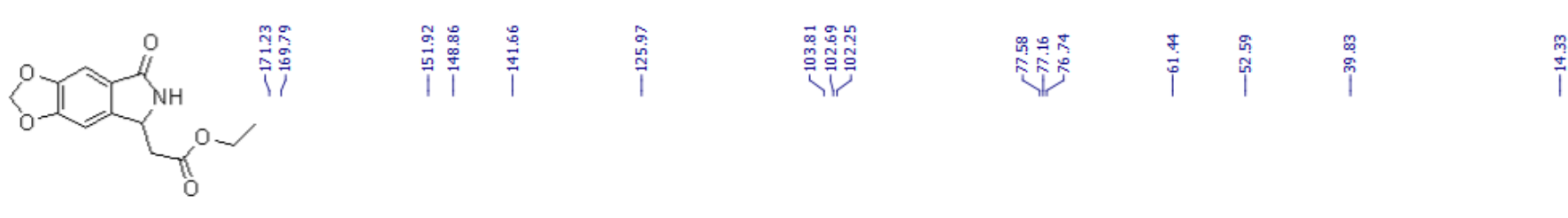

$75 \mathrm{MHz} C D C l_{3}$
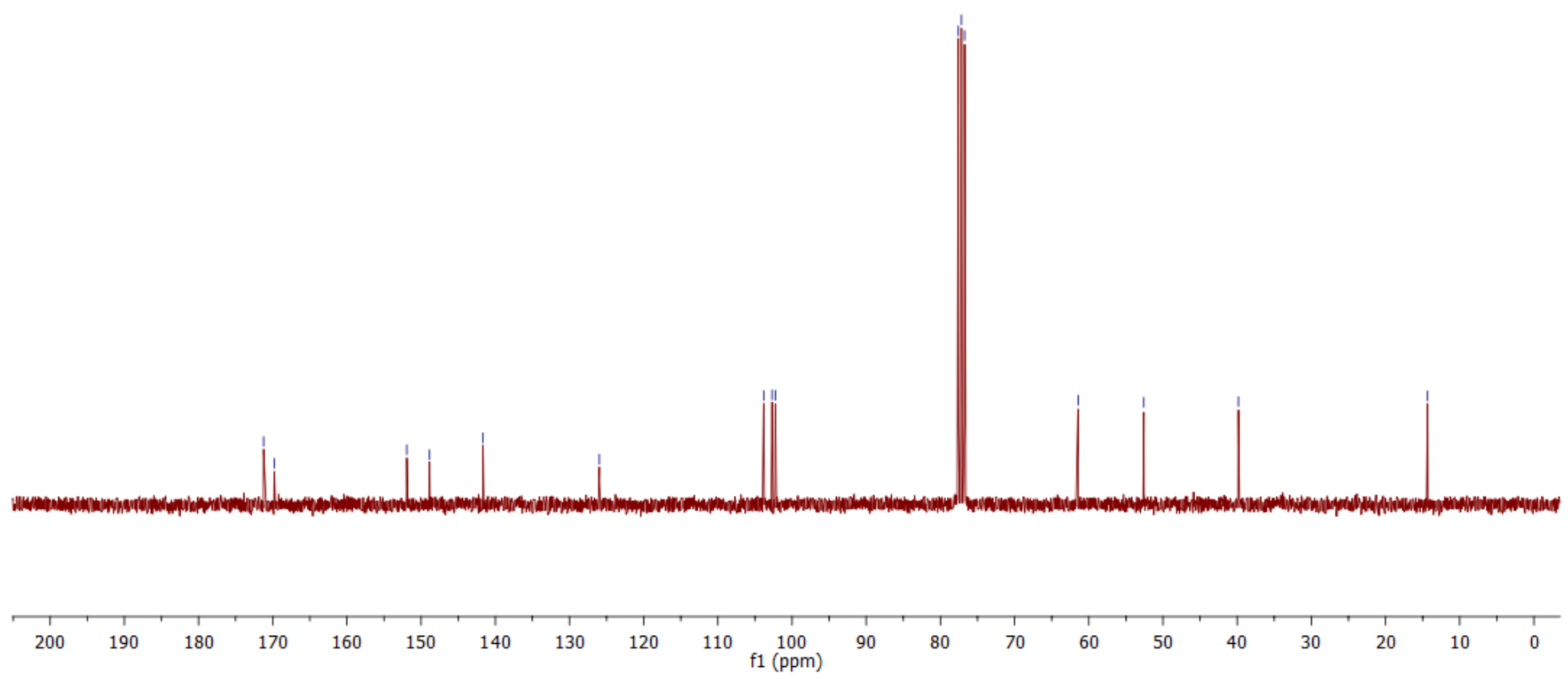
Methyl 2-(3-oxo-5-(trifluoromethyl)isoindolin-1-yl)acetate 15 c $300 \mathrm{MHz}\left(\mathrm{CDCl}_{3}\right)$

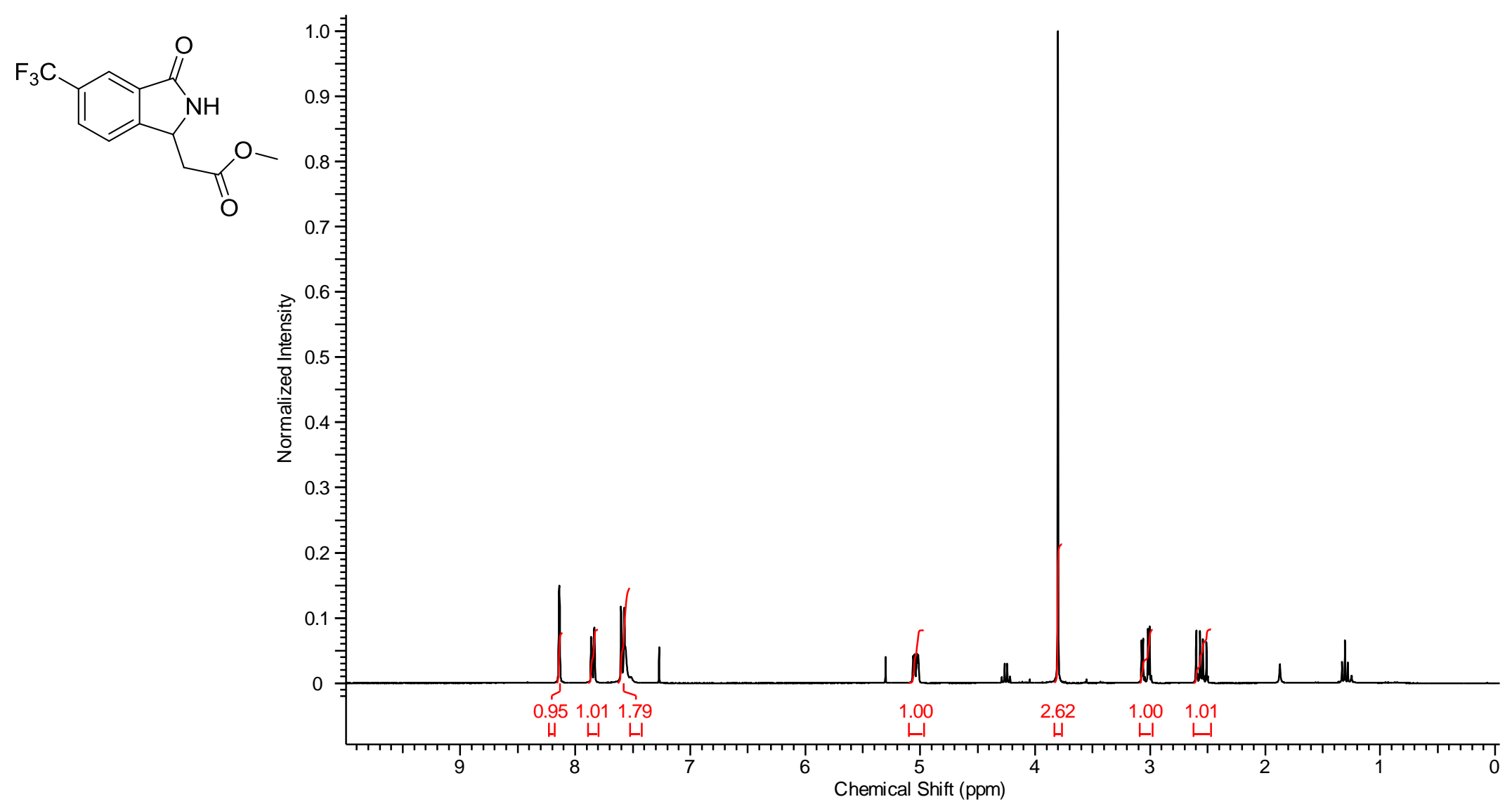


Methyl 2-(3-oxo-5-(trifluoromethyl)isoindolin-1-yl)acetate $15 \mathrm{c} 75.5 \mathrm{MHz}\left(\mathrm{CDCl}_{3}\right)$

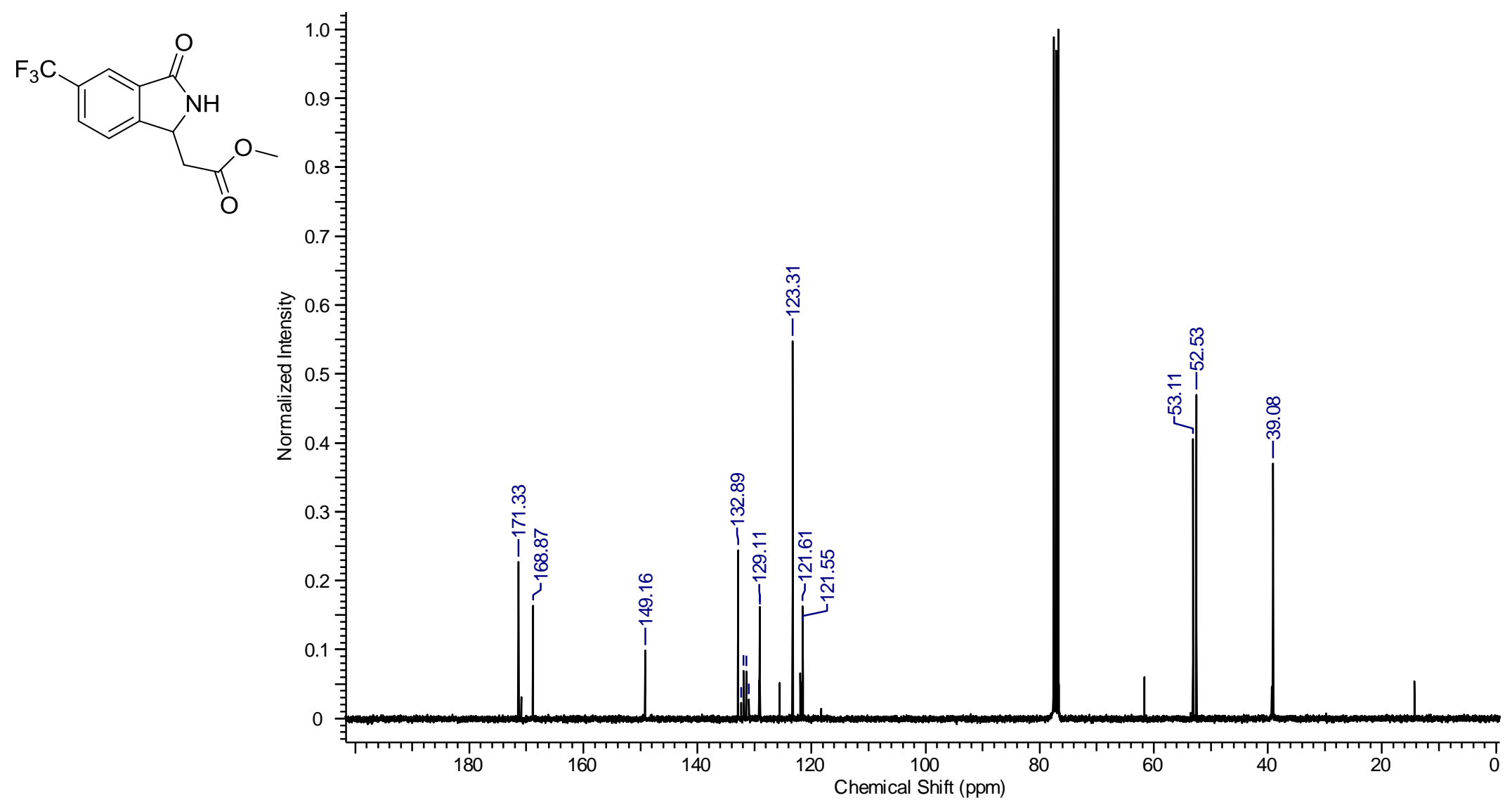


Methyl 2-(5-fluoro-3-oxoisoindolin-1-yl)acetate 15d

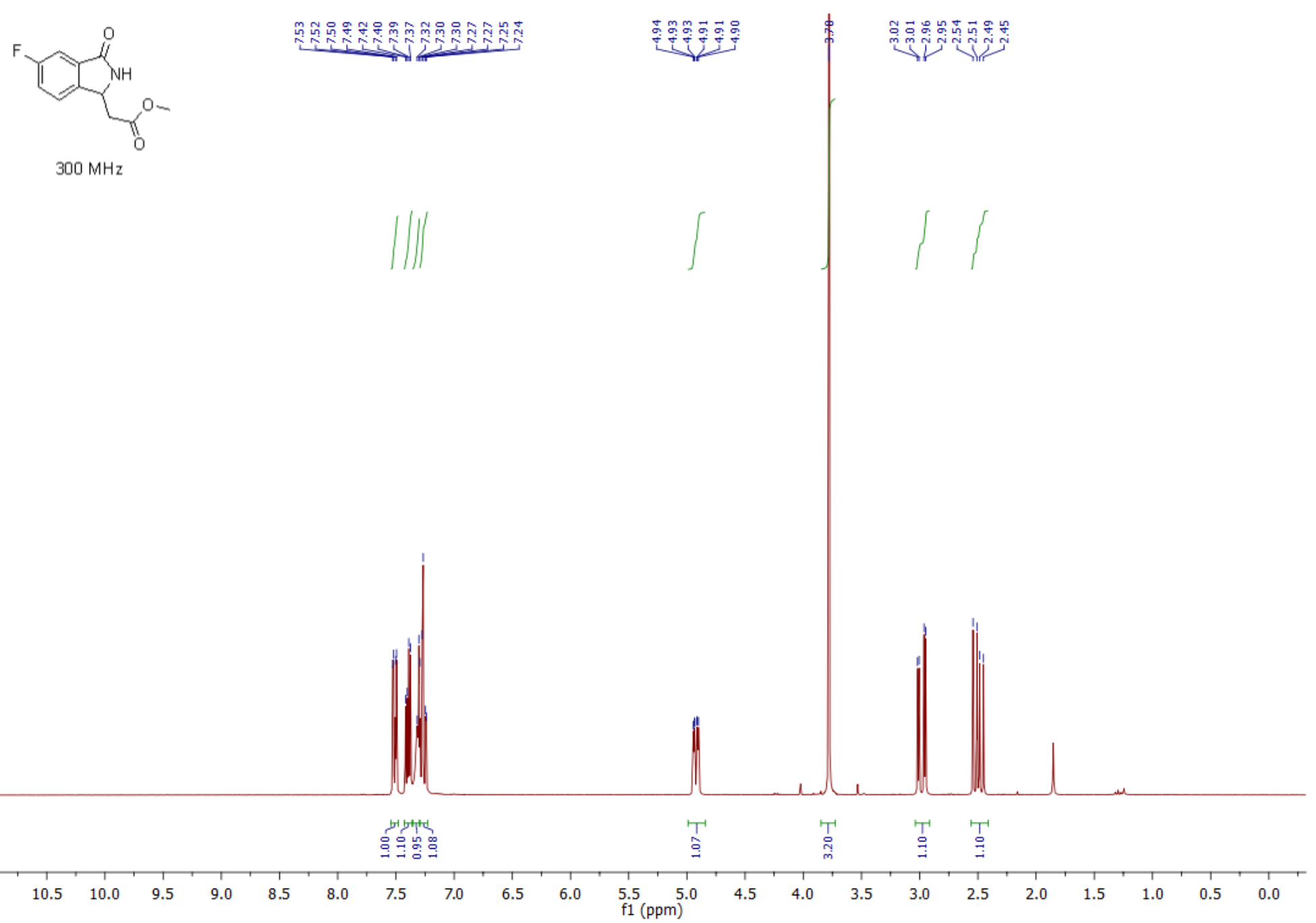


Methyl 2-(5-fluoro-3-oxoisoindolin-1-yl)acetate 15d
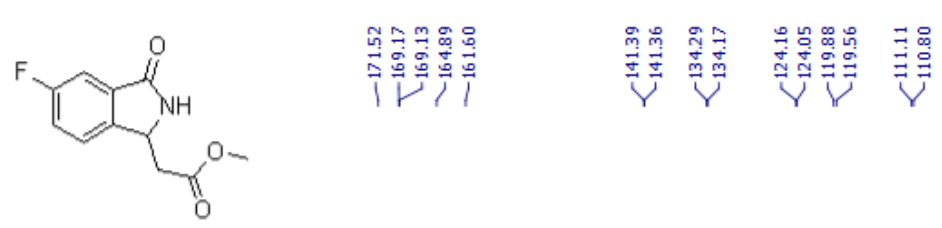

$75 \mathrm{MHz}$
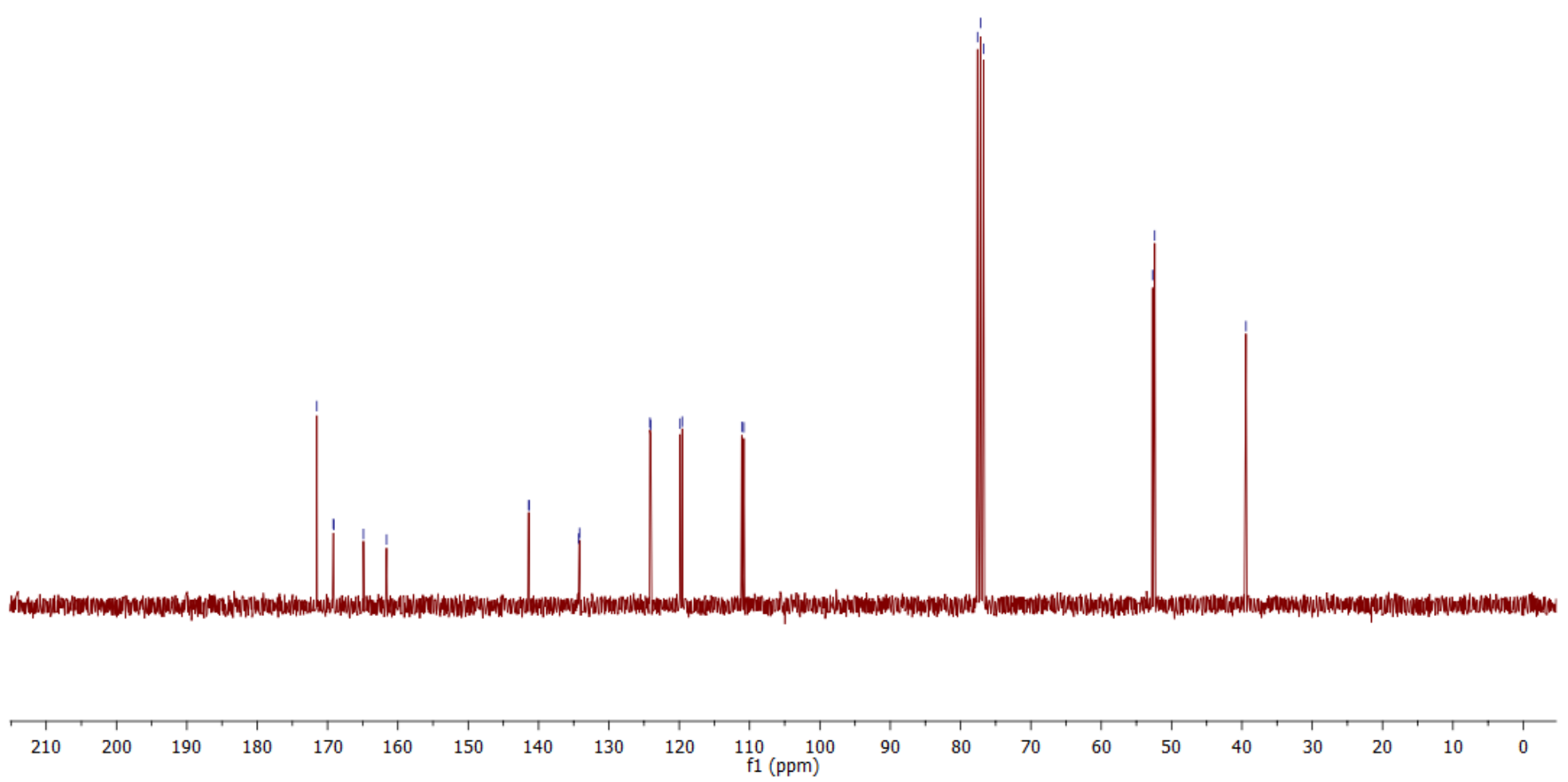
Methyl 2-(7-fluoro-1-oxoisoindolin-3-yl)acetate 15e
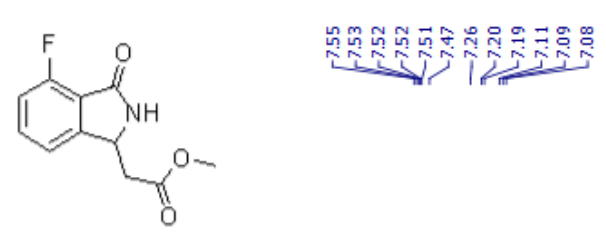

$500 \mathrm{MHz} \mathrm{CDCl}_{3}$
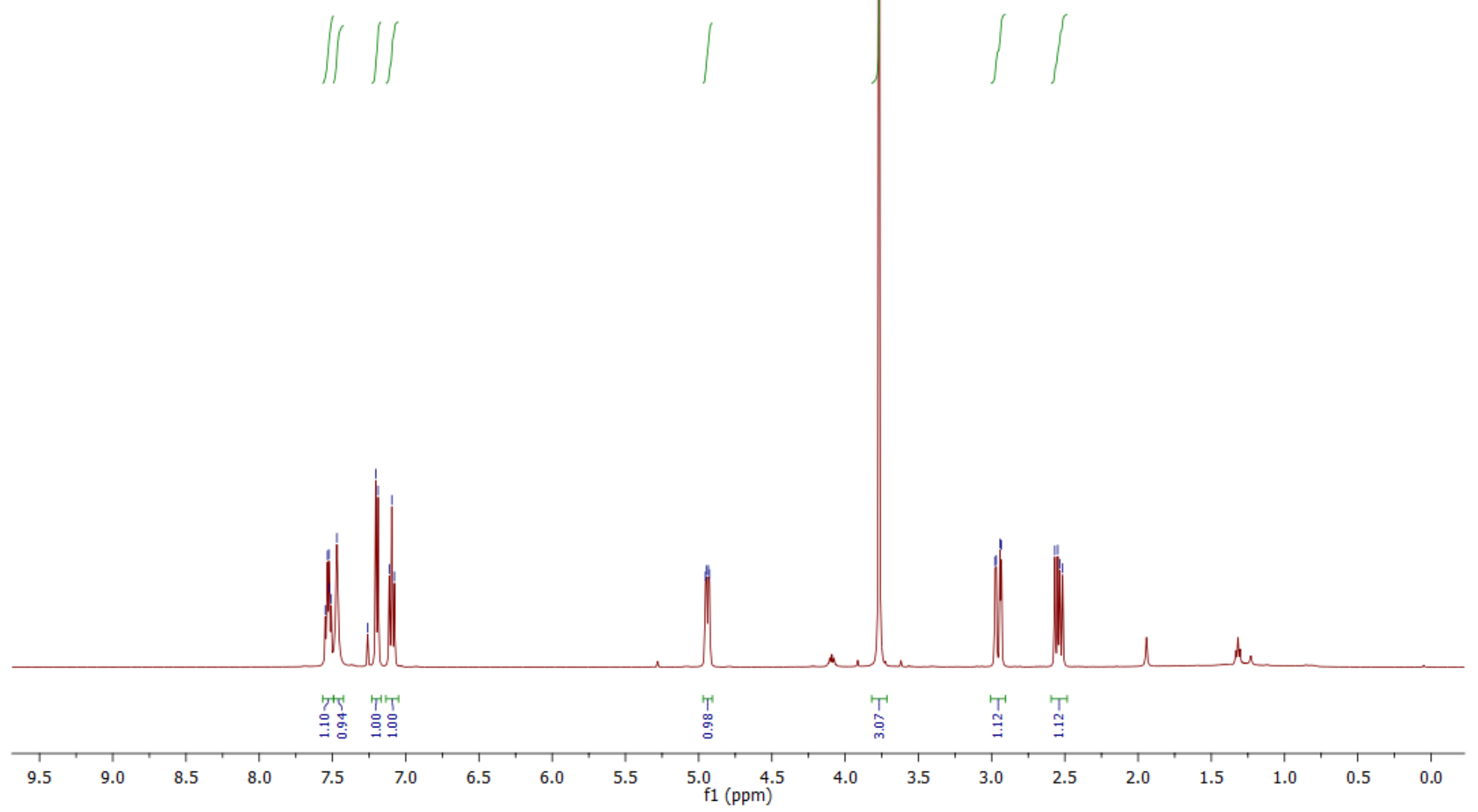
Methyl 2-(7-fluoro-1-oxoisoindolin-3-yl)acetate 15e

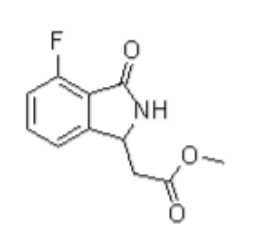

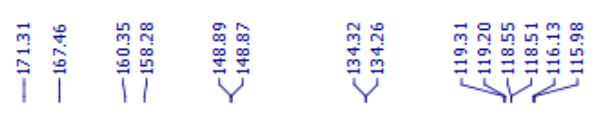

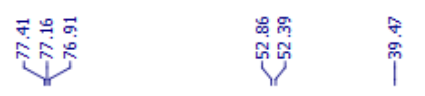

$126 \mathrm{MHz} \mathrm{CDCl}_{3}$

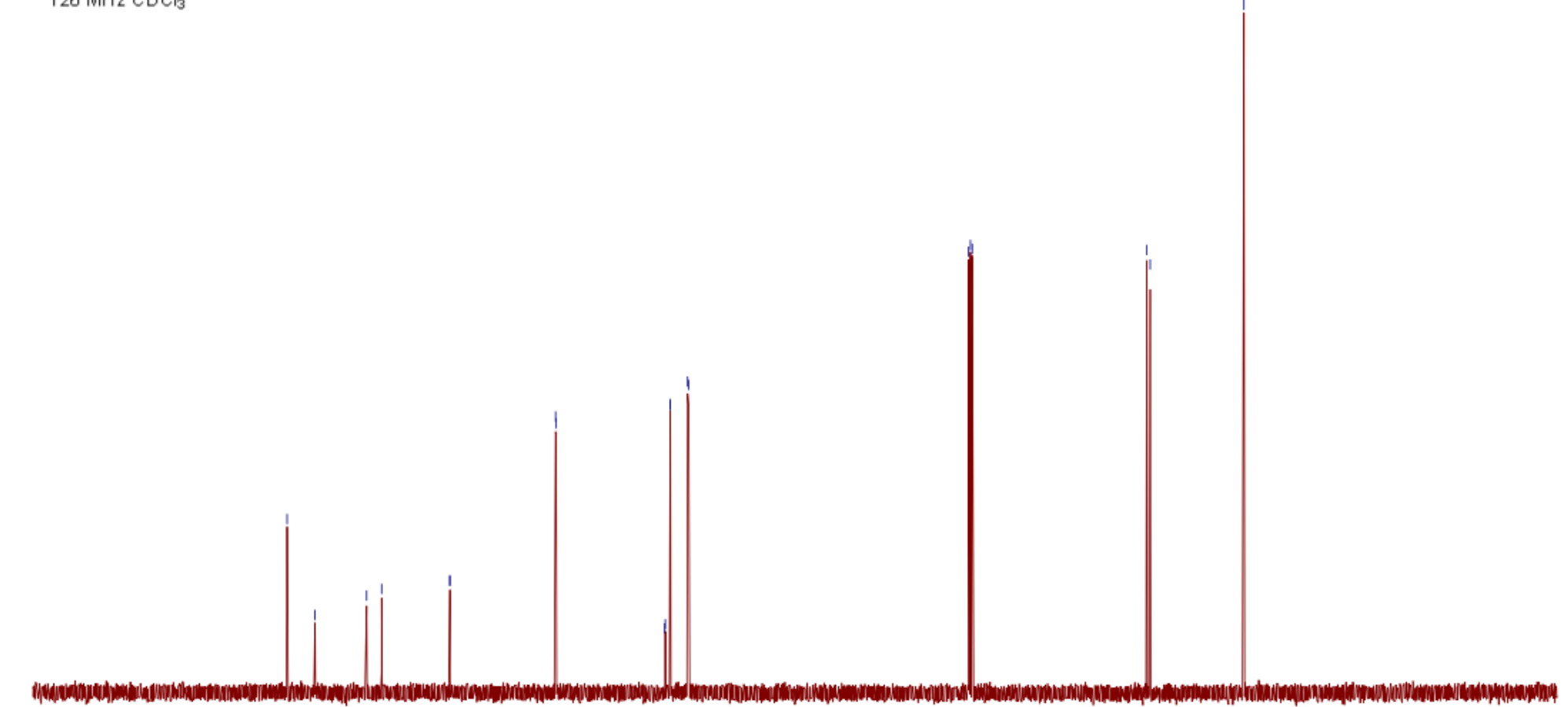


Tert-butyl 5-fluoro-1-(2-methoxy-2-oxoethyl)-3-oxoisoindoline-2-carboxylate 16

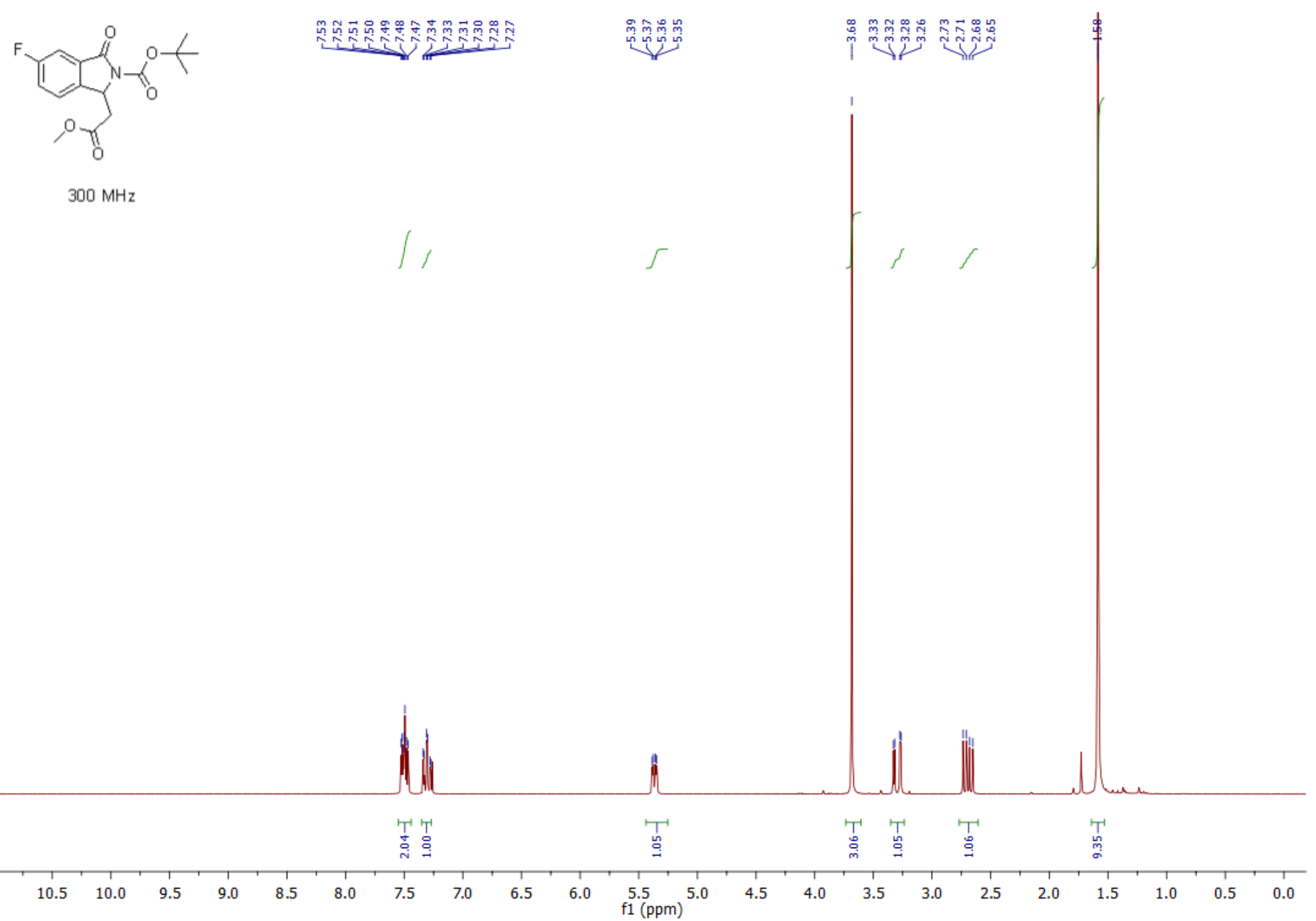


Tert-butyl 5-fluoro-1-(2-methoxy-2-oxoethyl)-3-oxoisoindoline-2-carboxylate 16
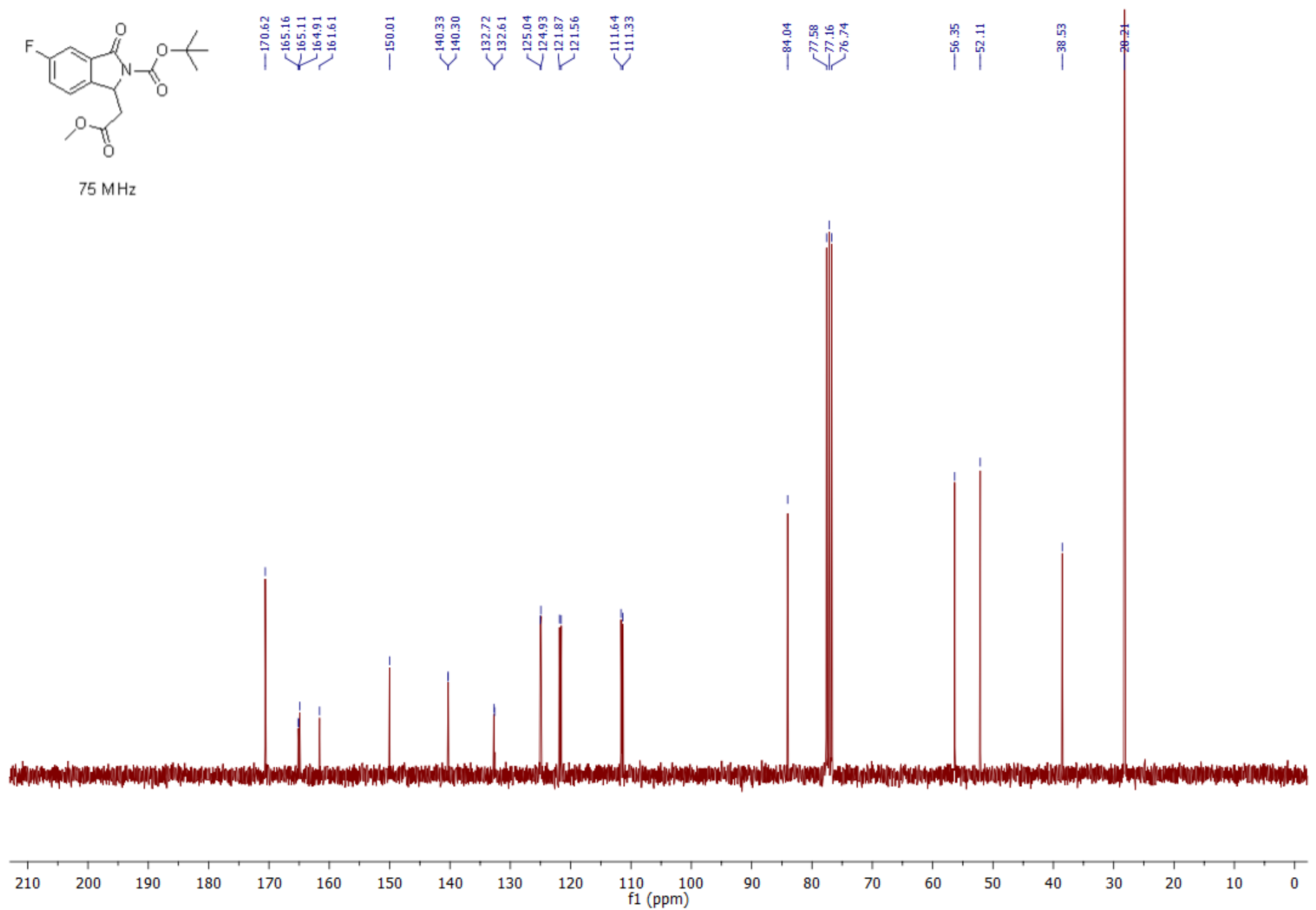
Tert-butyl 5-fluoro-1-(2-methoxy-2-oxoethyl)isoindoline-2-carboxylate $18300 \mathrm{MHz}\left(\mathrm{CDCl}_{3}\right)$

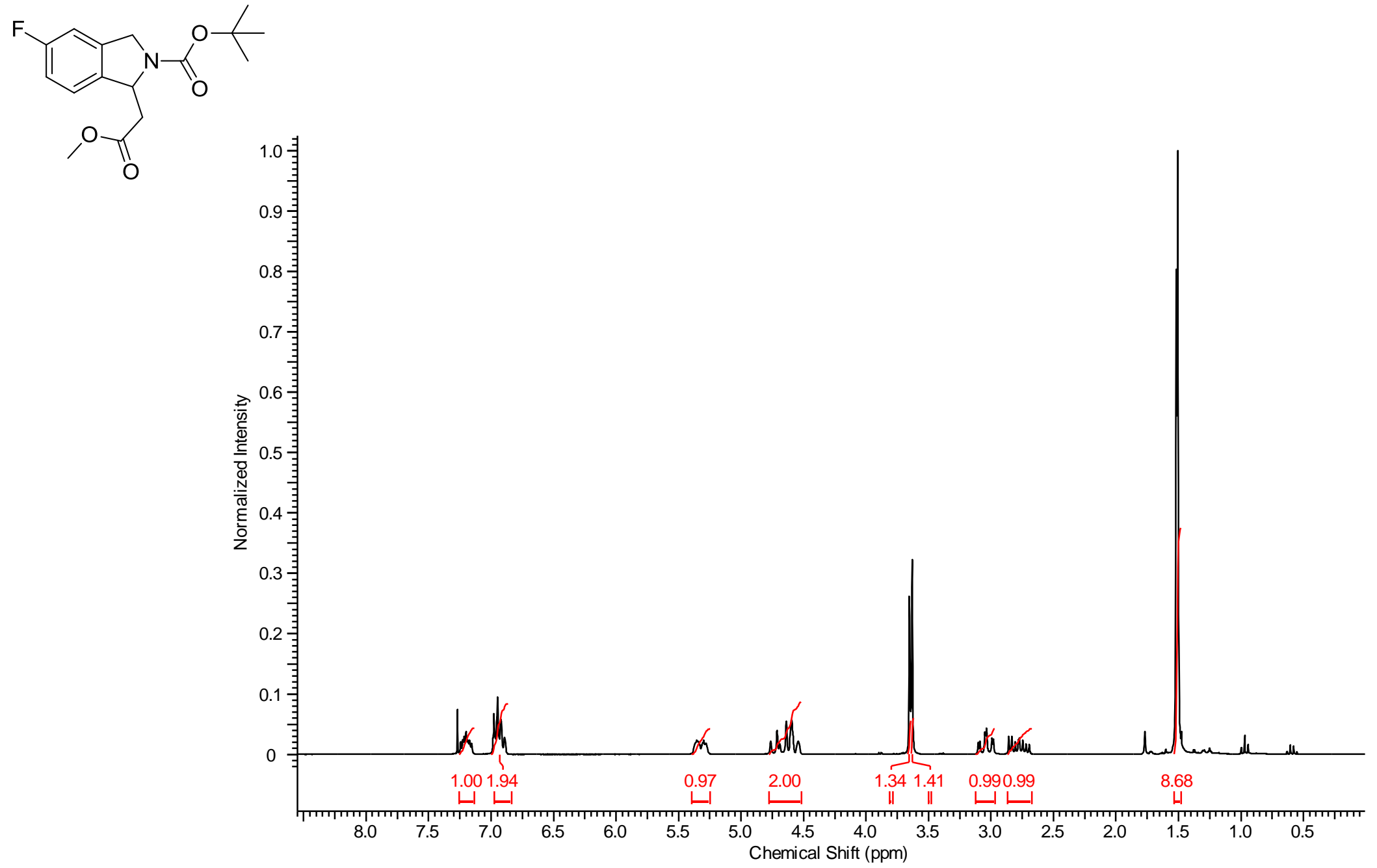


$500 \mathrm{MHz}\left(\mathrm{CDCl}_{3}\right)$ variable temperatura spctra of Tert-butyl 5-fluoro-1-(2-methoxy-2-oxoethyl)isoindoline-2-carboxylate 18

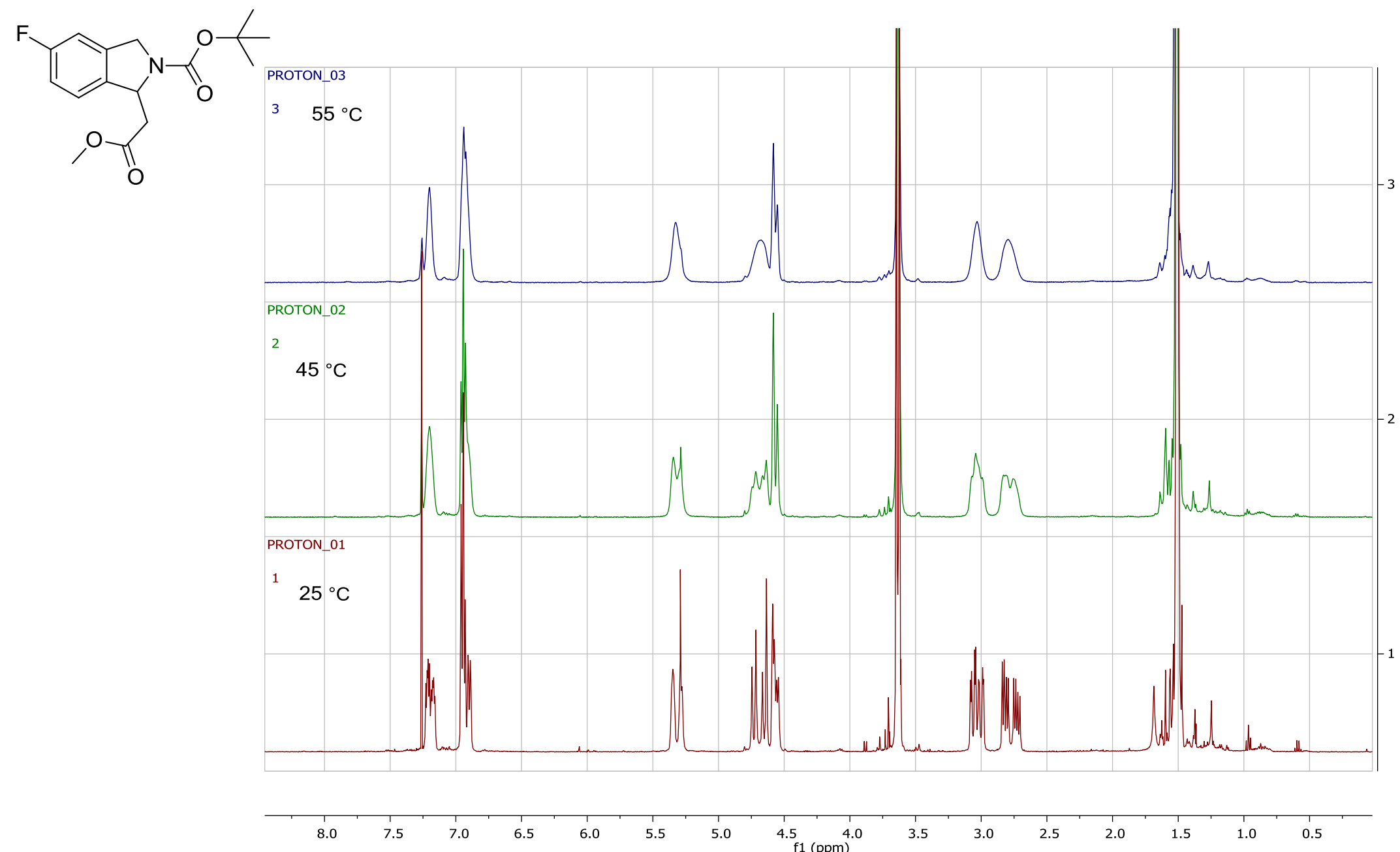


Tert-butyl 5-fluoro-1-(2-methoxy-2-oxoethyl)isoindoline-2-carboxylate $1875.5 \mathrm{MHz}\left(\mathrm{CDCl}_{3}\right)$

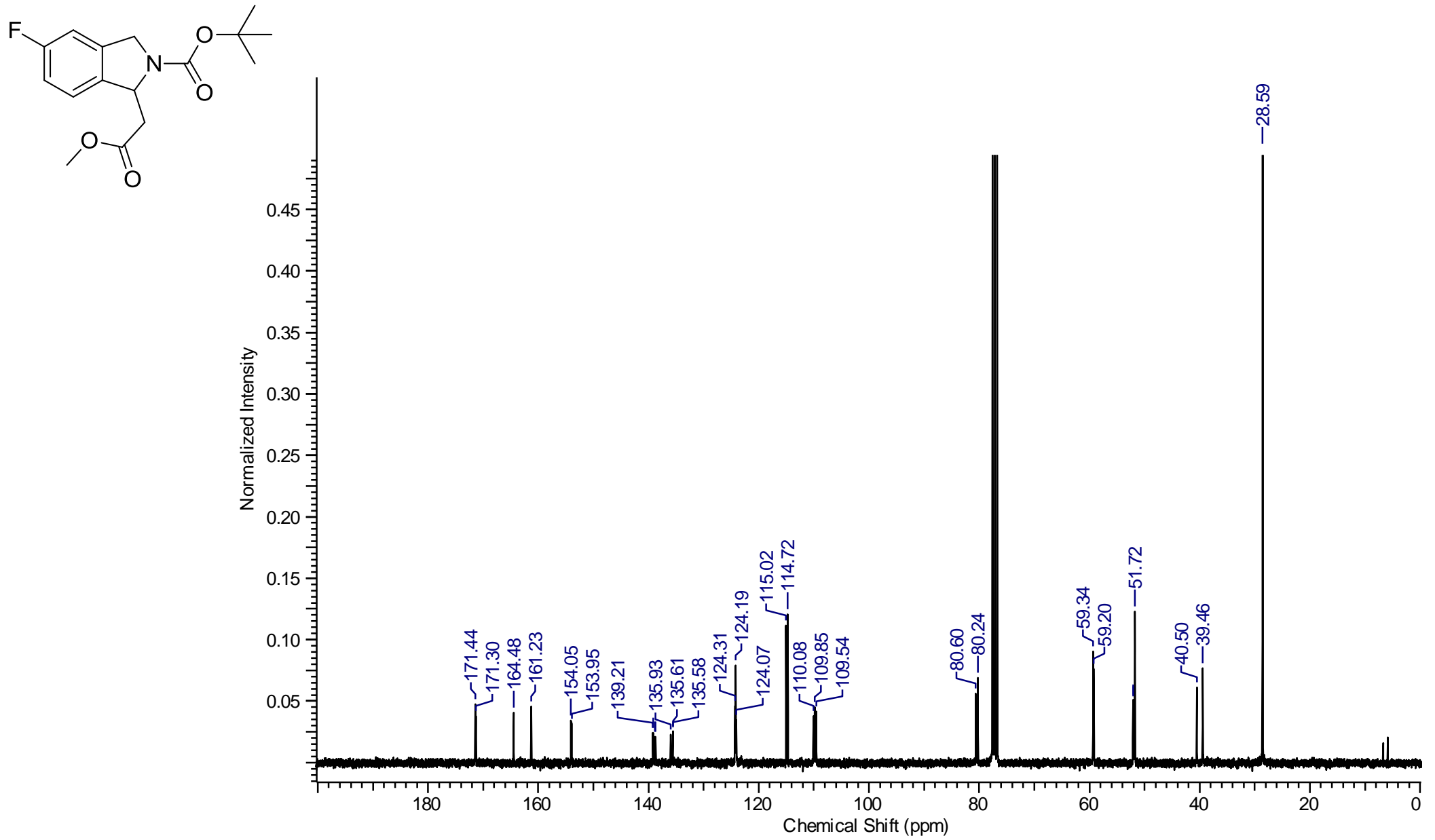


7-Fluoro-3-(2-hydroxyethyl)isoindolin-1-one 19

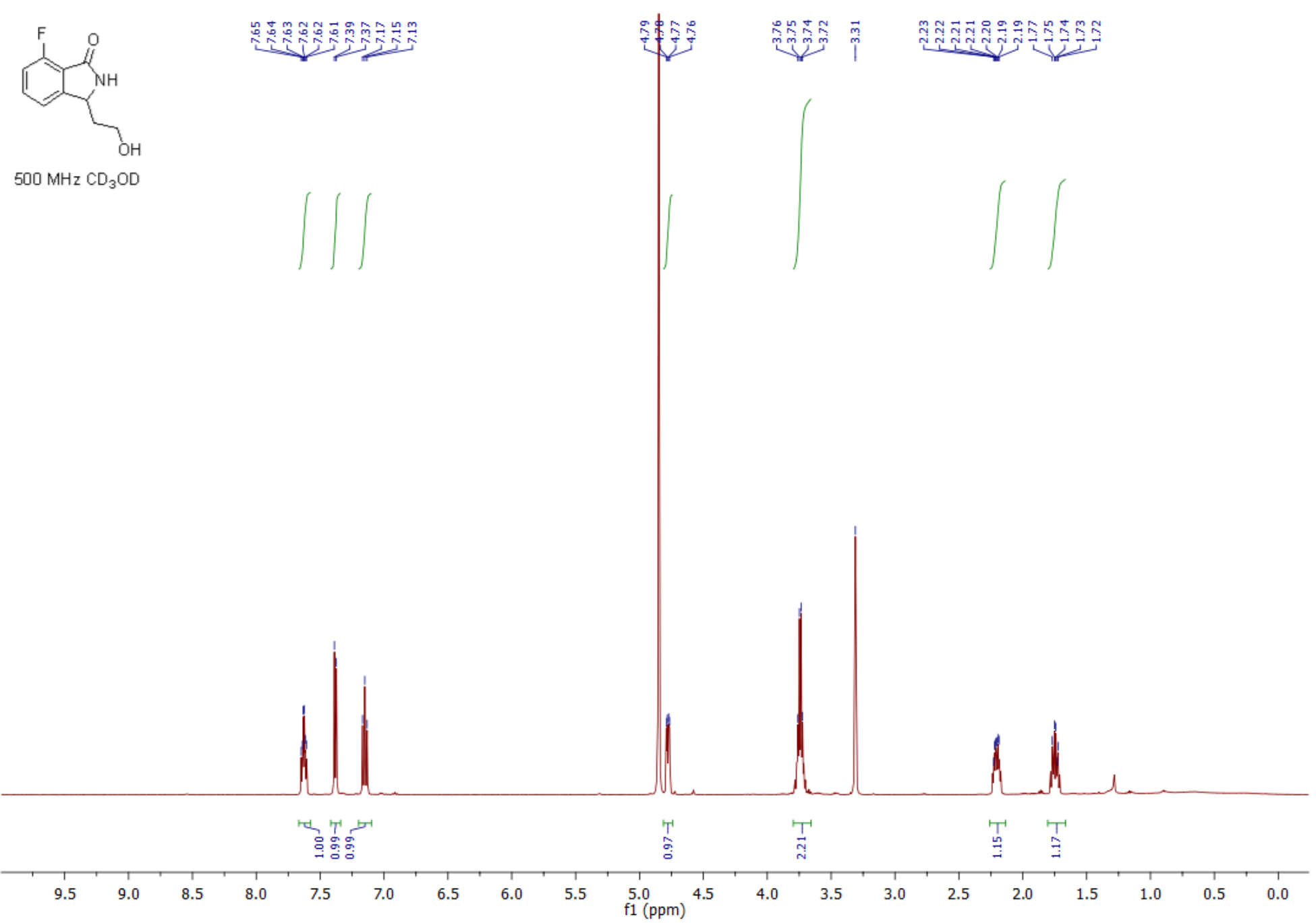


7-Fluoro-3-(2-hydroxyethyl)isoindolin-1-one 19
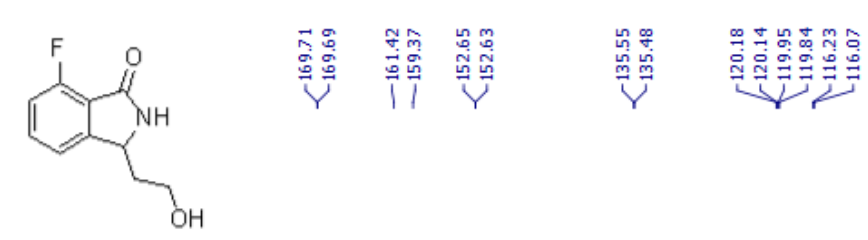

$126 \mathrm{MHz}_{\mathrm{CD}} \mathrm{OD}$
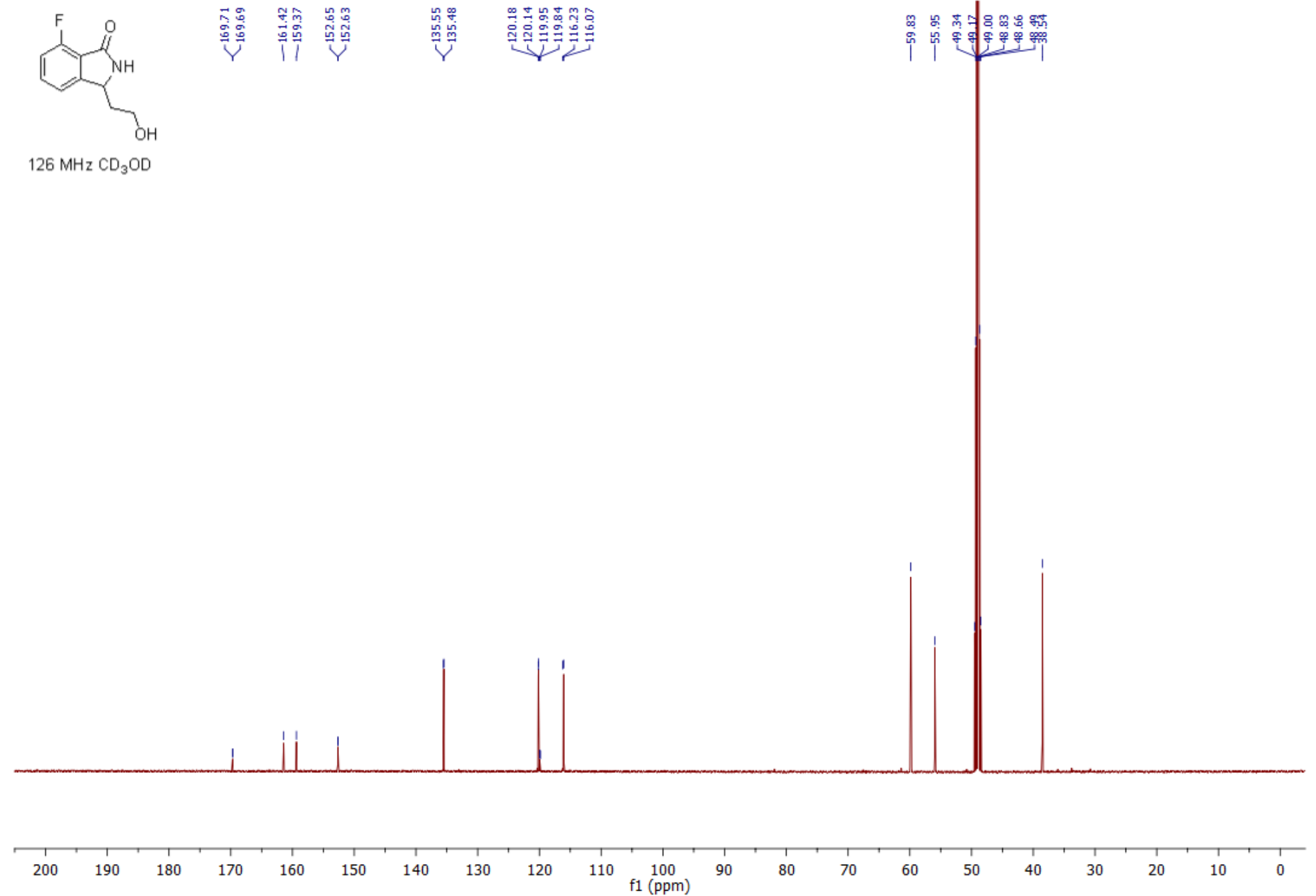
2-(4-fluoro-3-oxo-2,3-dihydro-1H-isoindol-1-yl)ethyl 4-methylbenzene-1-sulfonate 20

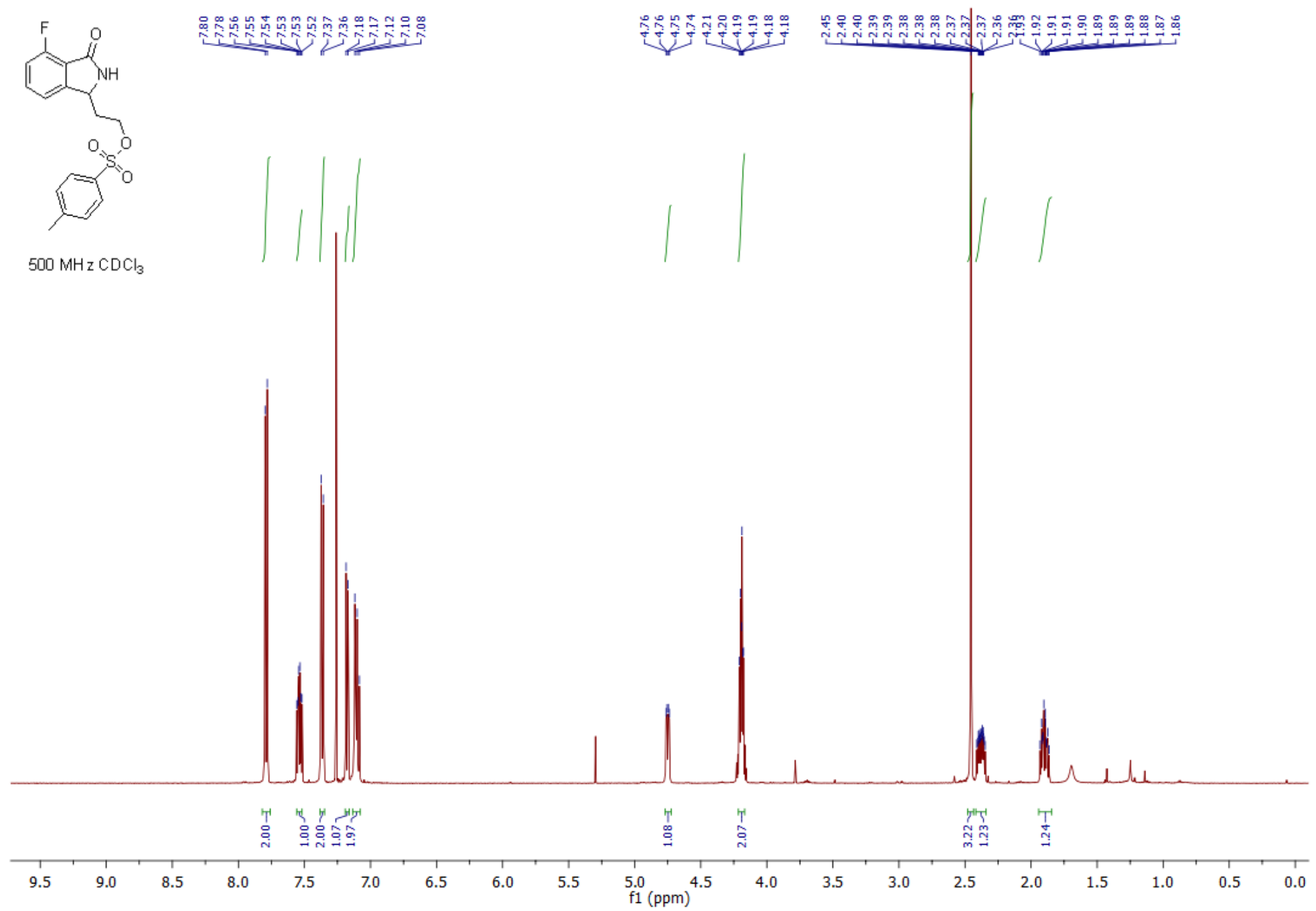


2-(4-fluoro-3-oxo-2,3-dihydro-1H-isoindol-1-yl)ethyl 4-methylbenzene-1-sulfonate 20
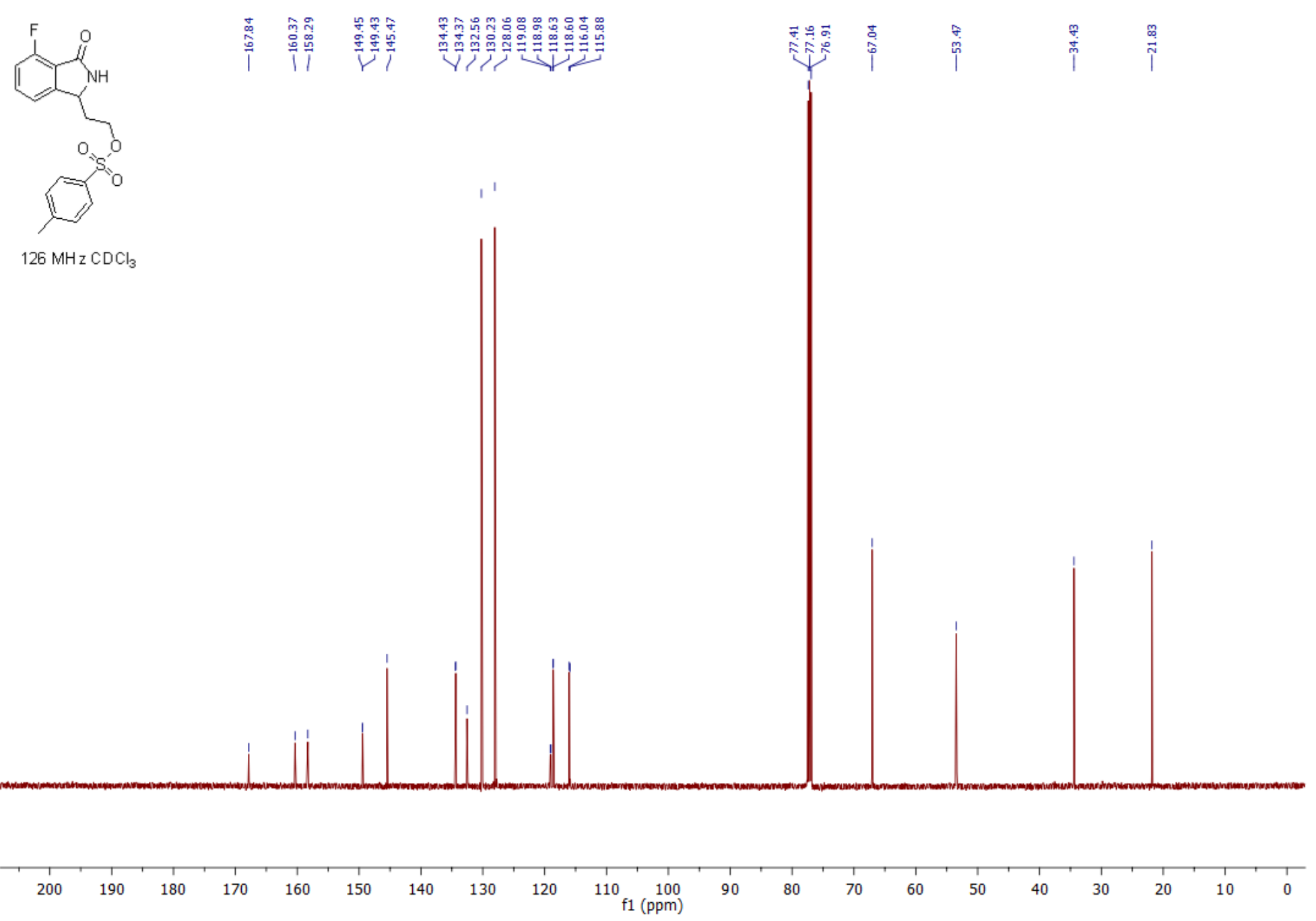
3-\{2-[4-(3,4-dimethylphenyl)piperazin-1-yl]ethyl\}-7-fluoro-2,3-dihydro-1H-isoindol-1-one 22
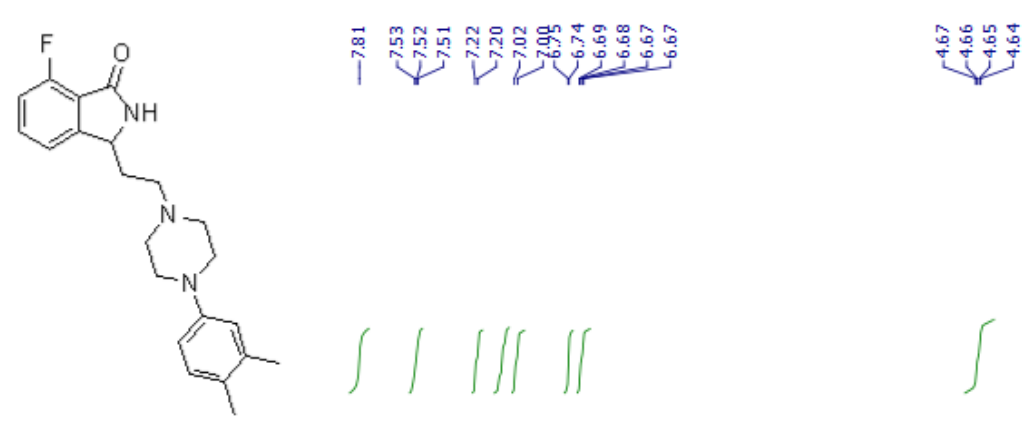

$500 \mathrm{MHz}_{2} \mathrm{CDCl}_{3}$

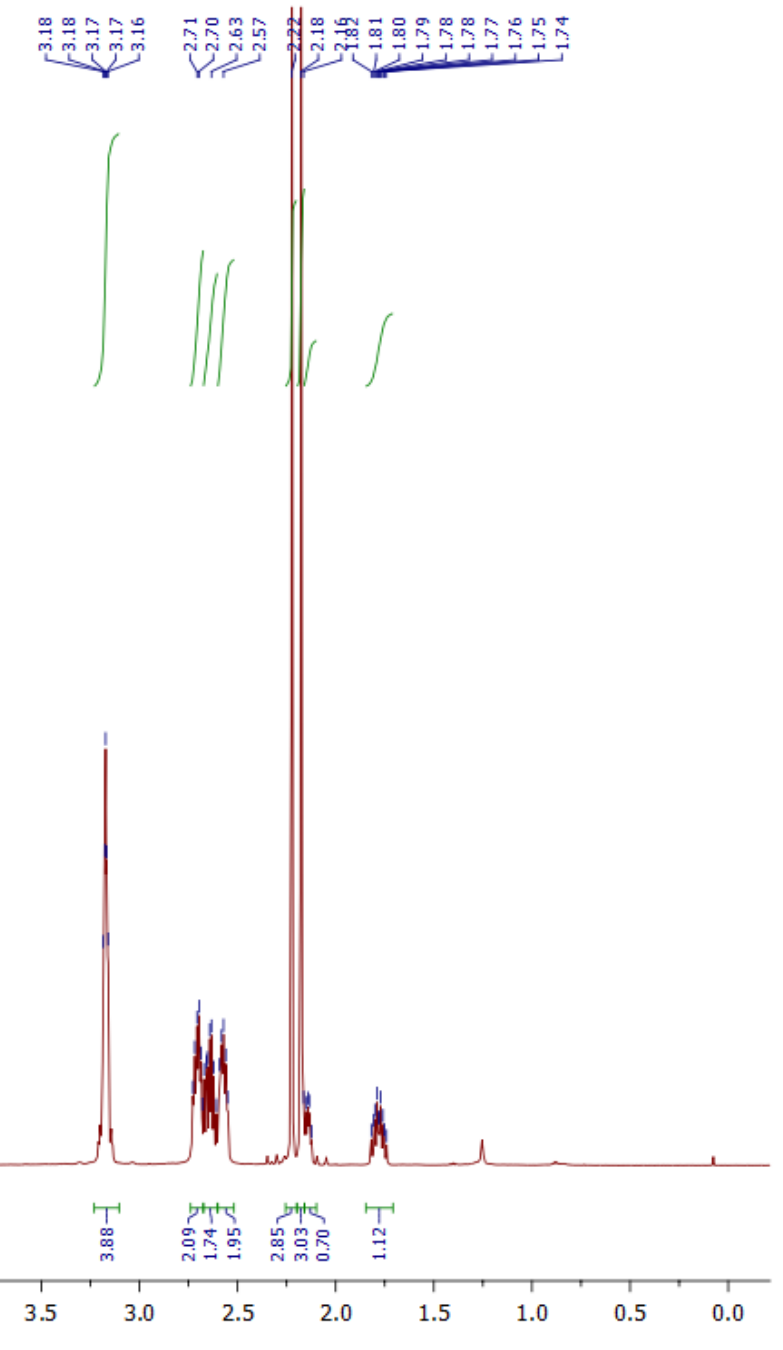


3-\{2-[4-(3,4-dimethylphenyl)piperazin-1-yl]ethyl\}-7-fluoro-2,3-dihydro-1H-isoindol-1-one 22
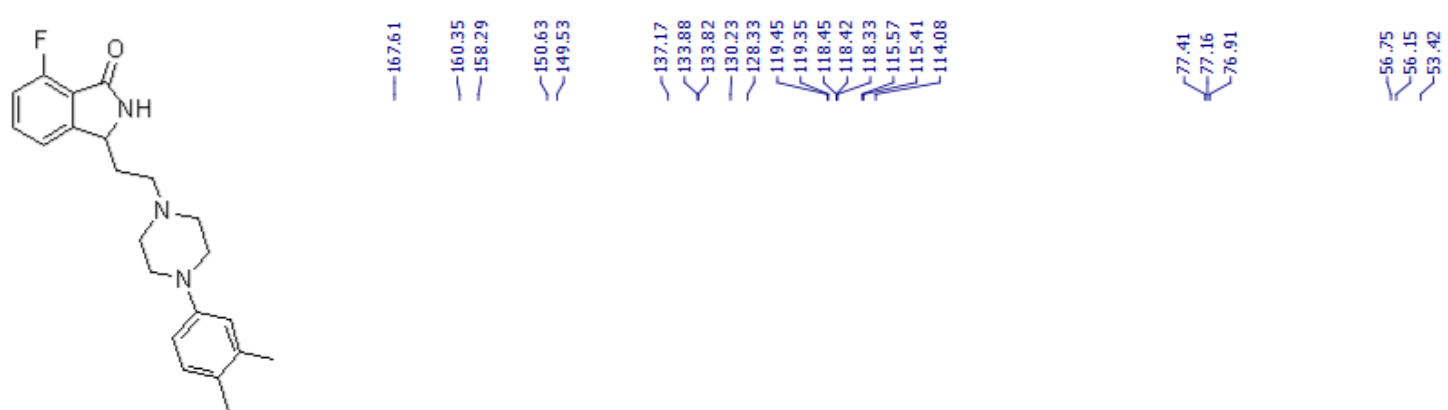

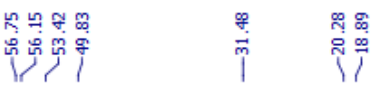

$126 \mathrm{MHz} \mathrm{CDCl}_{3}$

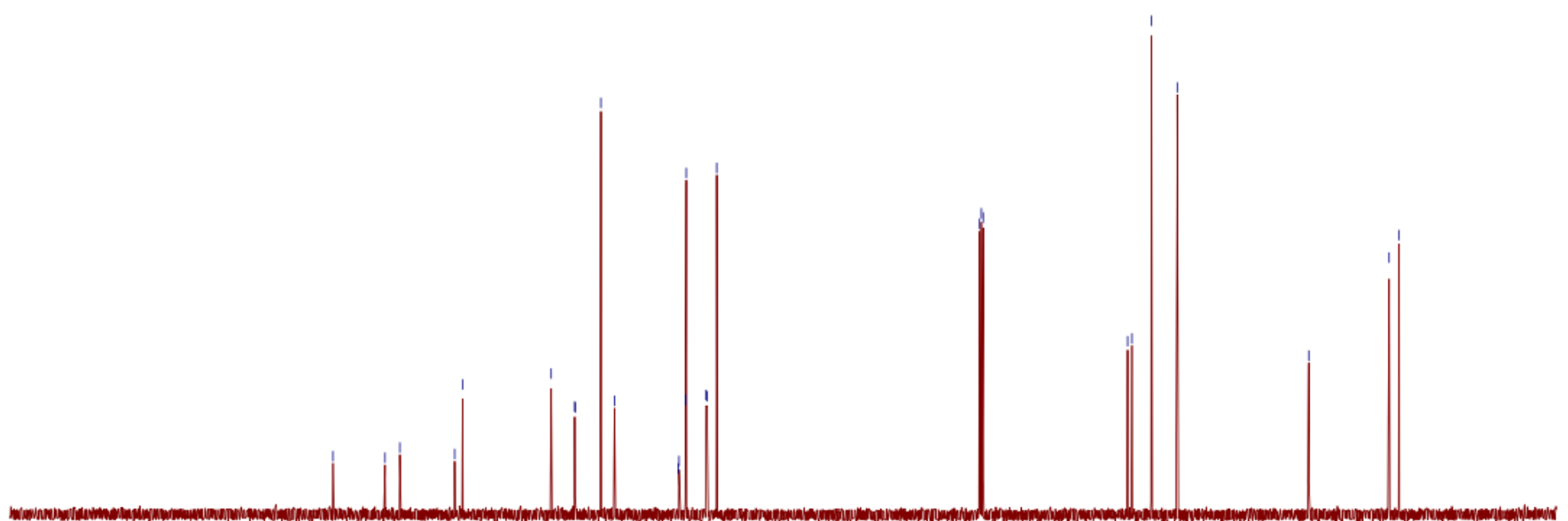

210
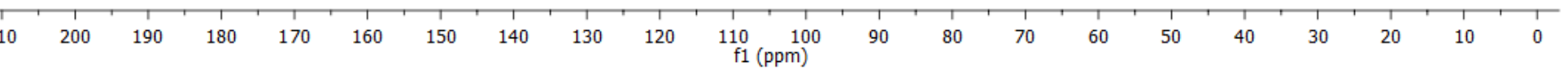